Portland State University

PDXScholar

Fall 1-1-2012

\title{
Causes and Consequences of Mitochondrial Variation in Caenorhabditid Nematodes
}

Kiley Ann Hicks

Portland State University

Follow this and additional works at: https://pdxscholar.library.pdx.edu/open_access_etds

Part of the Biology Commons, and the Other Genetics and Genomics Commons Let us know how access to this document benefits you.

\section{Recommended Citation}

Hicks, Kiley Ann, "Causes and Consequences of Mitochondrial Variation in Caenorhabditid Nematodes" (2012). Dissertations and Theses. Paper 928.

https://doi.org/10.15760/etd.928

This Dissertation is brought to you for free and open access. It has been accepted for inclusion in Dissertations and Theses by an authorized administrator of PDXScholar. Please contact us if we can make this document more accessible: pdxscholar@pdx.edu. 
Causes and Consequences of Mitochondrial Variation in Caenorhabditid

Nematodes

by

Kiley Ann Hicks

A dissertation submitted in partial fulfillment of the requirements for the degree of

\author{
Doctor of Philosophy \\ in \\ Biology \\ Dissertation Committee: \\ Suzanne Estes, Chair \\ Justin Courcelle \\ Jason Podrabsky \\ Todd Rosenstiel \\ Dirk Iwata-Reuyl
}

Portland State University 2012 
(C) 2012 Kiley Ann Hicks 


\begin{abstract}
Mitochondria are dynamic organelles that harbor their own stream-lined genome and generate much of the ATP necessary to sustain eukaryotic life via an electron transport chain (ETC). Because of the central role for mitochondria in organismal physiology, mitochondrial genetic and phenotypic variation can alter organismal fitness and affect population genetic and evolutionary outcomes. Despite the far-reaching relevance of mitochondria to evolutionary processes and human health, we lack a basic understanding of the evolutionary and cell biological causes and consequences of mitochondrial genetic and phenotypic variation. In this thesis, I quantified mitochondrial reactive oxygen species (ROS), membrane potential $(\triangle \Psi \mathrm{M})$, and mitochondrial morphological traits within Caenorhabditis briggsae natural isolates and lines exposed to extreme genetic drift (known as mutation-accumulation or MA lines) of both C. briggsae and Caenorhabditis elegans. Many natural isolates of C. briggsae are known to harbor mitochondrial DNA (mtDNA) variation in the form of a heteroplasmic nad5 gene deletion (nad5 $\Delta$ ) that correlates negatively with organismal fitness. The significant mtDNA variation among $C$. briggsae isolates provided an unprecedented opportunity to quantify the amount of mitochondrial phenotypic variation within a natural system. In Chapter two, statistically significant natural variation was observed for most mitochondrial form and function traits measured within a set of $C$. briggsae isolates. However, the associations between mitochondrial phenotypes and clade membership or nad5 level generally had very low $\mathrm{R}^{2}$ values,
\end{abstract}


meaning that only a minimal amount of the total variation in most of these traits could be attributed to nad5 $\Delta$ level or phylogeographic clade membership. In only one case $(\triangle \Psi \mathrm{M})$, clade membership accounted for nearly $50 \%$ of the observed phenotypic variation. Both ROS and $\triangle \Psi M$ levels of mitochondrial-nuclear hybrids, strains that contain the mitochondrial genome of one isolate on the nuclear background of another, were more similar to their mitochondrial parent suggesting that mtDNA variation underlies these traits. Hybrid analysis of the other seven important traits suggests both mtDNA and nuclear genetic variation as drivers of natural mitochondrial trait variation. Given the significant phenotypic variation among C. briggsae isolates observed in Chapter two, we next sought to determine whether natural selection might be shaping these important mitochondrial traits. In Chapter three, we used a mutationaccumulation approach to reduce the force of natural selection and found that average levels of both ROS and nad5 heteroplasmy evolved linearly in lines of C. briggsae natural isolates subjected to 20-50 generations of inbreeding. In particular, among $C$. briggsae isolates genetically-predisposed to the nad5 $\Delta$ deletion, nad5 $\Delta$ level increased to a plateau of $\sim 50 \%$ during successive generations of inbreeding. Conversely, mitochondrial ROS level increased or declined in a strain-specific fashion, resulting in no overall correlation between ROS and nad5 . In all cases, the isolate with the highest starting level of nad5 heteroplasmy went extinct prior to generation 20 of MA treatment, suggesting that nad5 $\Delta$ is detrimental at high heteroplasmy levels. Patterns of among-line variance in ROS level were also strain-specific but generally did not conform 
to the canonical pattern of increasing among-line variance expected for $M A$ experiments. While we uncovered significant variation in mitochondrial ROS, we found no correlation between ROS and mtDNA heteroplasmy level. Based on the vicious cycle hypothesis, it is expected that ROS will increase concomitantly with mutation rate. In Chapter four, we used a separate set of MA lines derived from C. elegans and that had previously been subjected to whole-genome sequencing to examine the relationship between oxidative stress and nuclear base substitution rate. MA lines were found to vary significantly in ROS levels but not in 8-oxo-dG content. Despite a significant positive correlation between 8-oxo-dG and ROS levels, no relationship between oxidative stress measures and base substitution rate or G-to-T transversion rate was revealed. Finding no relationship between ROS and mitochondrial or nuclear genetic variation, in Chapter four we analyzed a suite of 24 mitochondrial traits measured in C. briggsae natural isolates with the aim of identifying other mitochondrial phenotypes important for mitochondrial function and integrity. We uncovered several significant correlations between traits describing mitochondrial shape, number, and area and identified three consistent patterns of correlation: 1) mitochondria became bigger through elongation, 2) isolates with a more functional mitochondrial population (with relatively high overall $\Delta \Psi \mathrm{M})$ contained larger, more elongated mitochondria, and 3) isolates with a less functional mitochondrial population (with relatively low overall $\triangle \Psi \mathrm{M}$ ) contained a more heterogeneous mix of organelles. Individual $\triangle \Psi M$ level also correlated positively with the overall elongation of functional mitochondria, while ROS level did not correlate 
significantly to any measured traits. Using these relationships we developed a novel model of mitochondrial population dynamics wherein the functionality (relative $\Delta \Psi \mathrm{M}$ ) of the entire mitochondrial population will mediate individual organelle behavior. Specifically, individual mitochondria within more functional populations will be more likely to continually participate in fusion-fission cycles which will help to maintain a healthier population. Conversely, organelles in less functional populations will more often undergo autophagy, reducing the number of fusion capable mitochondria and creating shape heterogeneity in the entire population. This model could have implications for mitochondrial genome integrity. Overall, this work identifies extensive natural variation and capacity for evolution in mitochondrial traits within multicellular eukaryotic species, highlighting a central role for $\Delta \Psi \mathrm{M}$ in mitochondrial dynamics that may have implications for evolutionary adaptation to thermal niches. 


\section{DEDICATION}

For Griffin and Micah

"We must not cease from exploration. And the end of all our exploring will be to arrive where we began and to know the place for the first time." - T.S. Eliot 


\section{ACKNOWLEDGEMENTS}

I am deeply appreciative of the many people who directly and indirectly made this work possible. The Keith Garlid lab shared protocols and equipment, Aurelie Snyder (Advanced Light Microscopy Core Facility, OHSU) shared technical advice on confocal imaging and analysis, and Bart Braeckman provided generous input regarding worm protocols. Thanks also to Yangdong Pan for advice on classification tree analyses.

Several colleagues devoted hours of their time to this project and I am very thankful for their contributions. Many, many people outside of the Estes lab provided a great deal of support and advice; in particular I owe Jason Bazzanno, Isaac Sleadd, Amie Romney and Christine Sislak my gratitude. Gene De Haan devoted countless Sundays to the cause of isolating mitochondria, an endeavor that fails to even grace the following pages, but will not be forgotten. Many lab assistants poured hundreds of plates so that this work could take place, of which Noelle Al-Musaifry, Jenn Groody, and Sarah Welker deserve special thanks. Jasmina Dizdarevic provided laboratory assistance as well as assistance with analysis of ROS images for the C. briggsae MA lines. Nicole Brockway spent hours analyzing ROS images for the work described in Chapter three. Dana Howe, Rianna Wernick, and Aubrey Leung contributed to generating and nad5 $\Delta$ genotyping the many $C$. briggsae hybrid and mutation-accumulation lines used throughout this work. Dee Denver offered helpful advice and discussion for nearly every aspect of this project, as well as valuable revisions to the following chapters. Anna Coleman-Hulbert somehow managed to lend her support, knowledge, and useful sayings to me throughout my time 
at Portland State, and I am forever grateful to her. And to my sounding board and wing man, Sam Smith: my life and my science would not be the same without you.

Many thanks go to my extremely helpful and (at times) intimidating committee. I have developed a profound respect for each of you, not only as scientists but as people. The work presented here benefited greatly because you consistently challenged me to think about my research in unfamiliar ways. Plus, without your careful reading and insightful comments during my defense, I would be enjoying the sunshine right now instead of sitting at my computer. Thank you for reducing my risk of skin cancer in your own, unique way.

I am deeply indebted to Suzanne Estes, my research advisor and real-life Jack Donaghy. She has provided endless support and advice throughout the entirety of this work, from discussions regarding experimental design to statistical analysis to my choice in footwear. Her careful and thorough revisions have greatly improved the quality of this manuscript and all of my future writing. I cannot fully express the enormity or intensity of my gratitude for all that she has done for me.

I am grateful to the following funding agencies from which this work has benefited greatly: The National Institutes of Health grant 5 R01 GM087628-02 to DD and SE, the PSU Faculty Enhancement grant to SE, the American Heart Association Predoctoral Fellowship 11PRE4880069 to KAH, and the PSU Academically-Controlled Auxiliary Activities Committee to $\mathrm{KAH}$ and $\mathrm{ACH}$. 
Finally, I must thank my family. It is made up of my best friends and biggest champions. Many thanks go to Joel for absolutely everything. This includes, but is not limited to, putting up with hearing about my science endlessly and often and for making me laugh every day. 


\section{TABLE OF CONTENTS}

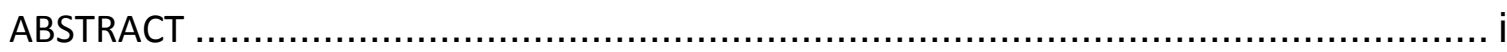

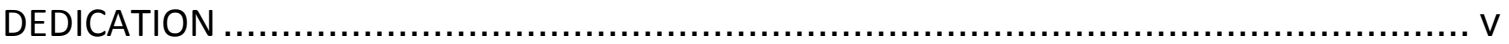

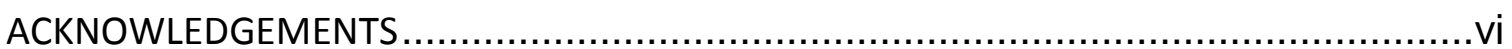

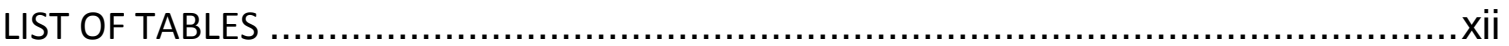

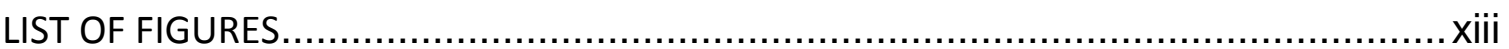

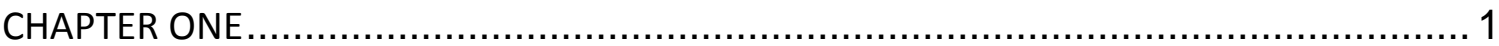

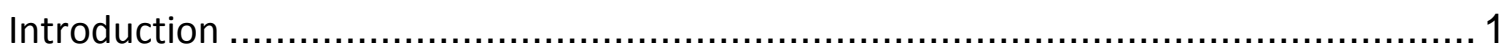

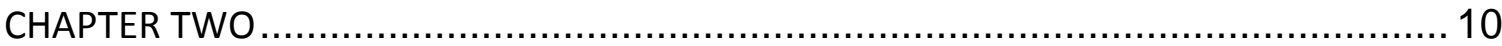

In vivo quantification reveals extensive natural variation in mitochondrial form and

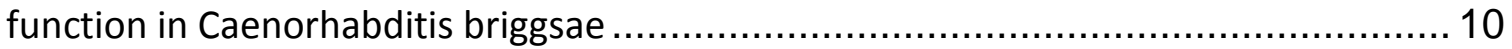

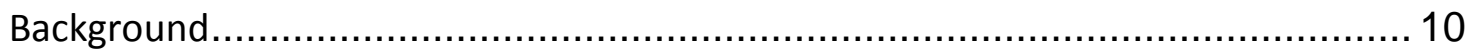

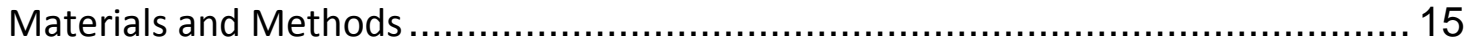

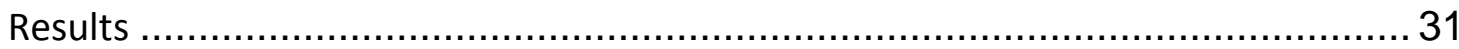

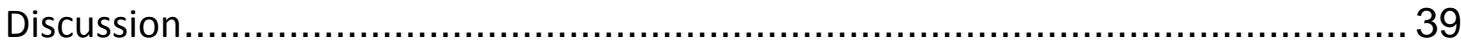

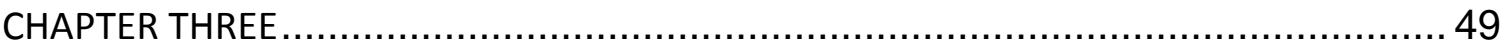

Evolution of mitochondrial heteroplasmy and ROS levels under genetic drift in experimental lines of Caenorhabditis briggsae ...................................................... 49 
Introduction

Materials and Methods

Results

Discussion

Reactive oxygen species level is related to 8-oxo-dG content but not germline base

substitution rate in Caenorhbaditis elegans

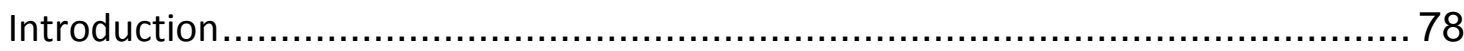

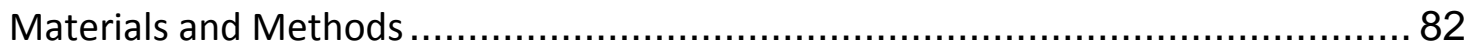

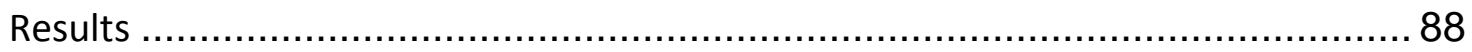

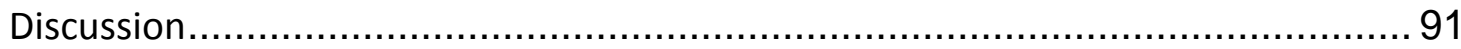

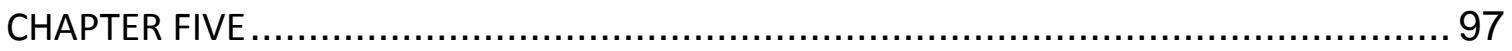

Natural variation in Caenorhabditis briggsae mitochondrial form and function suggests a

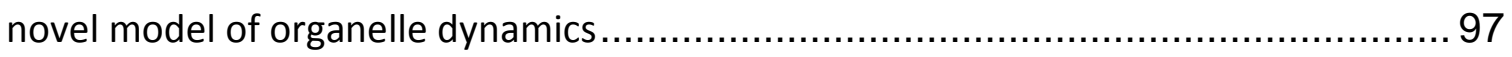

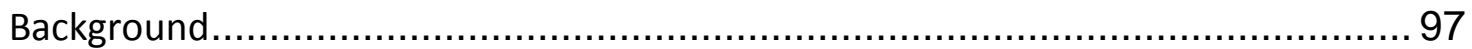

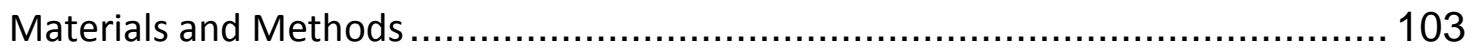

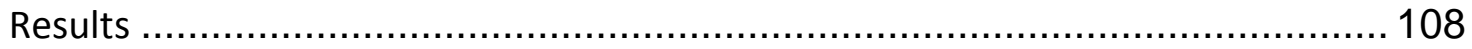

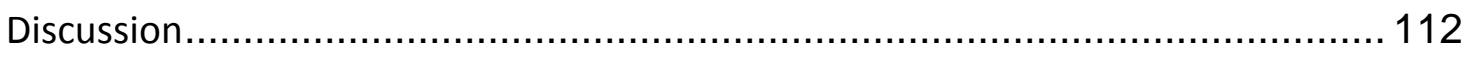




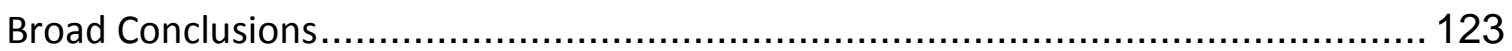

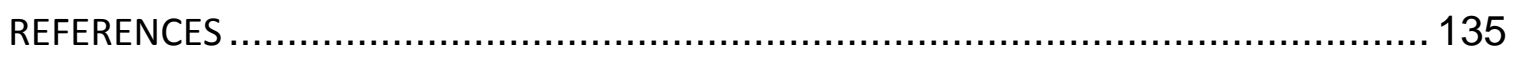

APPENDIX - Description of mitochondrial morphology analysis ........................... 157 


\section{LIST OF TABLES}

Table 2.1 Assigned labels and descriptions of all mitochondrial traits measured for $C$.

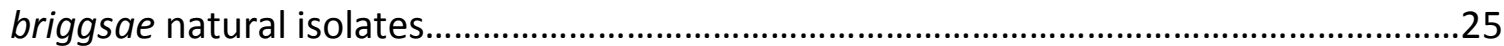

Table 2.2. Effect of phylogenetic clade and strain nested within clade for mitochondrial

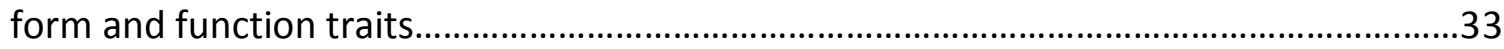

Table 2.3. Results of classification tree analysis.................................................................34

Table 2.4. Assigned labels of all mitochondrial traits measured for $C$. briggsae natural

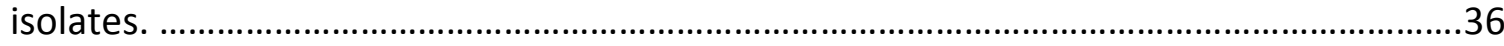

Table 2.5. Comparison of mitonuclear hybrid strains to parent strains.............................39

Table 3.1. Time to extinction and backup rates of $C$. elegans MA lines............................62

Table 3.2. Logistic model results for nad5 $\Delta$ deletion level variation among strains and generations. .65

Table 3.3. Chi-square results for strain-specific nad5 $\Delta$ differences between generational time points .66

Table 3.4. Two-way ANOVA results for ROS variation among strains and generations...67 Table 3.5. One-way ANOVA results describing among-line variation in ROS level at each time point

Table 4.1. Variation in Oxidative Stress-Related Traits.....................................................90

Table 5.1. Description of mitochondrial traits measured in C. briggsae .........................106

Table 5.2. Among-trait correlations in C. briggsae isolates............................................114 


\section{LIST OF FIGURES}

Figure 2.1. Natural and experimental C. briggsae strains and description of the nad5 $\Delta$ mtDNA deletion.

Figure 2.2. Localization of mitochondria-targeted dyes in C. briggsae nematodes..........20

Figure 2.3. Associations between mitochondrial function and morphology traits and isolate-specific nad5 $\Delta$ level.

Figure 2.4. ROS and $\triangle \Psi \mathrm{M}$ in mitonuclear hybrid strains...................................................38

Figure 3.1. C. briggsae natural isolates and description of the nad5 $\Delta$ mtDNA deletion...56

Figure 3.2 Evolution of nad5 $\Delta$ mtDNA levels under mutation accumulation treatment...63

Figure 3.3. Evolution of average nad5 $\Delta$ level, relative ROS level, and the correlation between ROS and nad5 $\Delta$ levels over MA... .64

Figure 3.4. Variance in net ROS levels before and after 20-50 generations of extreme inbreeding. .69

Figure 4.1 - Quantification of relative in vivo ROS levels in C. elegans terminal pharyngeal bulbs. 89

Figure 4.2. Bivariate relationship of MA line means for net in vivo ROS level and 8-oxodG content. .91

Figure 5.1. C. briggsae natural isolates. 101

Figure 5.2. Examples of bivariate relationships of mitochondrial phenotypes

Figure 5.3. A context-dependent model of mitochondrial dynamics in which mitochondria respond to the functional state of their intracellular environment. 118 


\section{CHAPTER ONE}

Introduction

Mitochondria are highly dynamic cellular organelles responsible for producing a majority of the ATP used to fuel eukaryotic life through the process of oxidative phosphorylation at the electron transport chain (ETC). Within the ETC, the passage of electrons down their energy gradient through a series of functional protein complexes acts to pump protons across the inner mitochondrial membrane, generating an electrochemical potential known as the mitochondrial membrane potential $(\triangle \Psi \mathrm{M})$. This $\triangle \Psi \mathrm{M}$ fuels the production of ATP and appears to control mitochondrial dynamics (Twig et al. 2008a) as well as the rate of production of potentially damaging reactive oxygen species (ROS) byproducts by the ETC (Murphy 2009). Mutations affecting ETC genes that impair oxidative phosphorylation are associated with a variety of human metabolic and agerelated disorders (Wallace 2005a; Biskup and Moore 2006) and often result in alterations to ROS production (Verkaart et al. 2007; Dingley et al. 2009), $\triangle \Psi M$ (Ventura et al. 2006; Gaskova et al. 2007; Dingley et al. 2009; Lemire et al. 2009), and mitochondrial morphology and dynamics (Lee et al. 2003; Pham et al. 2004; DuvezinCaubet et al. 2006). Because these functions are deeply interrelated and critical for mediating important aspects of organismal physiology, mutations affecting just one of these traits may induce pleiotropic effects at the level of the cell, tissue, or whole organism. Accordingly, ETC variation has been implicated as a major player in 
evolutionarily important processes such as aging (Balaban et al. 2005; Lemire et al. 2009; Kowald and Kirkwood 2011) and speciation (Ballard and Whitlock 2004; Willett and Burton 2004; Burton et al. 2006, 2007; Gershoni et al. 2009; Keller and Seehausen 2012). Despite the importance of mitochondrial function for such diverse biological issues as human disease and evolution, we still lack basic information on the levels of standing mitochondrial phenotypic variation and of the complex relationships between genetic variation and organelle function and dynamics, as well as the potential causes and consequences of natural variation therein.

In addition to housing the ETC, mitochondria also possess a small, streamlined genome (mtDNA) whose gene products cooperate with those encoded in the nucleus to maintain proper ETC function. Laboratory mutation-accumulation (MA) experiments which reduce the power of selection by maintaining replicate lineages (MA lines) under extreme inbreeding thereby allowing mutations to accrue (Halligan and Keightley 2009) - have revealed mtDNA mutation rates $~ 10 x$ higher than nuclear rates and show evidence for variation in the underlying mutational processes among and within species (Denver et al. 2000; Haag-Liautard et al. 2008). Many features of mitochondrial biology appear to promote high rates of deleterious mutation accumulation in mtDNA compared to the nuclear genome, including proximity to damaging oxygen radicals (Ballard and Whitlock 2004). When mutated, mitochondrial genomes tend to exist in a heteroplasmic state, wherein wild type mitochondrial genomes coexist alongside mutant genomes within the same organism, tissue, cell, or organelle (Lightowlers et al. 
1997; Chinnery et al. 2000). The presence of heteroplasmy provides the opportunity for mitochondrial threshold effects wherein a certain fraction of mutant mitochondrial genomes must be present for deleterious phenotypes to manifest (Rossignol et al. 2003; Ventura et al. 2006). It has been suggested that the smaller size of deletion-bearing mitochondrial genomes relative to the intact (wild type) genomes in a heteroplasmic organelle may confer a replicative advantage for the mutant genome (Moraes 2001; Diaz et al. 2002); recent work supports this idea (Clark et al. 2012). Evidence comparing nucleotide polymorphism across taxa with a wide range of population sizes suggests that purifying selection is shaping mtDNA (Bazin et al. 2006; Meiklejohn et al. 2007). However, we have little information on the contribution of ROS production to evolutionarily important mutation accumulation. Conversely, few studies have attempted to evaluate the evolutionary forces potentially affecting mtDNA heteroplasmy (Denver et al. 2000; Haag-Liautard et al. 2008) and mitochondrial phenotypes such as ROS.

Mitochondria are a major source of endogenous ROS. As a likely consequence, eukaryotic cells have developed various antioxidant defense mechanisms to combat ROS accumulation to levels that can damage nucleic acids, proteins, and lipids (Sedensky and Morgan 2006; Imlay 2008). However, mitochondrial ETC dysfunction and impairment can induce oxidative stress, wherein an increase in ROS production rates leads to a disproportionately high oxidant load compared to the cellular antioxidant capacity (Grad and Lemire 2004; Halliwell and Gutteridge 2007). Excessive mitochondrial 
ROS production has been associated with the accumulation of somatic mitochondrial and nuclear mutations in several age-related human diseases (Klaunig and Kamendulis 2004; Wallace 2005a). Such observations are in line with the free radical theory of aging proposed by Denham Harman in 1956, which states that the lifetime of exposure to unavoidable reactive byproducts (including ROS) of the ETC will damage important macromolecules causing age-related deterioration (Harman 1956). The vicious cycle theory expands upon this idea, proposing that the oxidative damage incurred by DNA, proteins, and lipids will further reduce mitochondrial performance causing an exponential increase in mitochondrial ROS production (Bandy and Davison 1990). However, mounting evidence suggests that the mutational processes of the soma and the germline may be quite different (Drake et al. 1998; Fortune 2000; Martorell et al. 2000; Shanks et al. 2008; Crabbe and Hill 2010; Lynch 2010; Joyner-Matos et al. 2011), and relatively few studies have examined the role of ROS in propagating heritable germline mutation (Denver et al. 2006; Stoltzfus 2008; Joyner-Matos et al. 2011).

It is becoming clear that factors other than mitochondrial ROS could be vitally important to maintaining mtDNA genome integrity. We now understand that mitochondria participate in continuous cycles of fusion, fission, and autophagy that link organelle shape to mitochondrial function (Chen and Chan 2005; Duvezin-Caubet et al. 2006), and each mitochondrion to the larger organelle population (Hyde et al. 2010). These cycles are essential for maintaining mitochondrial function, as demonstrated by the observation that abnormal fusion-fission cycles are characteristic of 
neurodegenerative disorders including Parkinson's and Alzheimer's disease (Trimmer et al. 2000; Wang et al. 2008; Irrcher et al. 2010; Su et al. 2010; Winklhofer and Haass 2010). Further, studies in isolated human cells have revealed a direct relationship between $\triangle \Psi M$ and mitochondrial morphology, such that higher $\Delta \Psi M$ induces organellar elongation (Legros et al. 2002; Ishihara et al. 2003) and loss of $\Delta \Psi M$ causes severe fragmentation of the mitochondrial network (Duvezin-Caubet et al. 2006; Song et al. 2007). Twig, et al. (2008b) proposed a mitochondrial "quality control axis" governed by $\Delta \Psi \mathrm{M}$ and carried out via the fusion-fission-apoptosis cycle to maintain mitochondrial integrity (Twig et al. 2008b). Their model states that mitochondria with insufficient $\Delta \Psi M$ are incapable of fusion and are thereby segregated from the functional mitochondrial population (Twig et al. 2008a). Using the "litmus test" of $\Delta \Psi M$, dysfunctional organelles - and perhaps, damaged mitochondrial genomes - are removed from the population allowing the persistence of an overall healthier mitochondrial population (Twig et al. 2008b; Hyde et al. 2010; Kowald and Kirkwood 2011; Bess et al. 2012; Meyer and Bess 2012). Despite the recent upsurge of research examining individual mitochondrial dynamics, relatively little work has focused on the population-level behaviors of these organelles. In particular, we have essentially no understanding of how population-level mitochondrial dynamics might influence individual organelles. Further, it is unclear whether mitochondrial dynamics research conducted using isolated cells and genetic mutants will translate into live animals and natural populations of organisms. 
Caenorhabditid nematodes have emerged as an important model system for studying the underlying causes of mitochondrial ETC dysfunction and its associated biological consequences, from examinations of the cellular and genetic underpinnings of the oxidative stress response (Grad and Lemire 2004; Kayser et al. 2004; An et al. 2005; Inoue et al. 2005; Lee et al. 2010; Yasuda et al. 2011) to genome-wide rates and patterns of spontaneous mutation (Denver et al. 2000, 2006, 2009). The small size, highly differentiated tissues and transparent cuticle of nematodes make them ideal for live imaging studies. Caenorhabditids have a short life-cycle and high wildtype fecundity that make them valuable for multi-generation evolutionary studies (Denver et al. 2000, 2006; Ajie et al. 2005; Baer et al. 2005; Estes et al. 2005). Also, worms and mammals share remarkably similar mitochondrial metabolism and ETC function (Dimmer et al. 2002; Westermann 2010). Finally, Caenorhabditids exhibit a cosmopolitan distribution and C. briggsae harbors a great deal of mitochondrial genetic and phenotypic diversity (Howe and Denver 2008; Cutter et al. 2010; Raboin et al. 2010; Estes et al. 2011; Ross et al. 2011; Clark et al. 2012; Hicks et al. 2012). Specifically, geographically segregated natural isolates of $C$. briggsae are known to contain a large mitochondrial genome deletion (named nad5 $\triangle$ ) of the $\mathrm{NADH}$-dehydrogenase 5 (nad5) gene, which encodes an important component of the ETC (Howe and Denver 2008). nad5 is thought to result from recombination events between nad5 and an upstream pseudogene ( $\Psi$ nad5-2) (Lunt and Hyman 1997), which means that Kenyan isolates without $\Psi$ nad5-2 are incapable of experiencing the deletion while $C$. briggsae isolates with $\Psi$ nad5-2 
experience nad5 $\Delta$ at heteroplasmy levels ranging from zero to over $50 \%$. A good deal of work indicates that aspects of nematode health and fitness may suffer when nad5 $\Delta$ heteroplasmy levels exceed $\sim 40 \%$ (Howe and Denver 2008; Estes et al. 2011). Further, mitochondrial genetic analyses divide $C$. briggsae isolates into three major phylogeographic clades (Cutter et al. 2006; Howe and Denver 2008) that are likely adapted to local temperature regimes (Jovelin and Cutter 2011; Prasad et al. 2011). The substantial mitochondrial genetic and phenotypic variation within natural isolates of $C$. briggsae and laboratory mutants of Caenorhabditis elegans, combined with their amenability to live imaging and experimental evolution make Caenorhabditid nematodes an ideal system with which to study the causes and consequences of mitochondrial variation.

To explore the genetic causes and evolutionary consequences of mitochondrial phenotypic variation, I aimed to 1) quantify the amount of mitochondrial phenotypic variation within natural populations of eukaryotes, 2) determine the role of natural selection in shaping this variation, and 3) identify mitochondrial traits important for genetic integrity by assaying mitochondrial ROS, $\triangle \Psi \mathrm{M}$, and a number of traits describing mitochondrial morphology and population structure within natural isolates of $C$. briggsae and mutation-accumulation lines of $C$. elegans and C. briggsae. In Chapter two, I uncovered significant variation in eighteen mitochondrial traits among ten natural isolates of C. briggsae, much of which was explained by phylogeographic clade membership; this was particularly true for $\Delta \Psi \mathrm{M}$, which was a reliable predictor of clade 
membership. Phenotypic similarities between two mitochondrial-nuclear hybrid strains and their mitochondrial parent strains suggest that mtDNA variation contributes to the expression of $\triangle \Psi M$ and ROS levels. Finally, I discuss these findings in light of evidence for local thermal adaptation in C. briggsae. In Chapter three, I observed a significant evolutionary capacity for mitochondrial ROS and nad5 $\Delta$ levels in MA lines generated from natural isolates and one hybrid strain of $C$. briggsae. Both ROS and nad5 5 evolved in a strain-specific manner during MA treatment, with ROS evolution following a highly linear trajectory in the natural isolates, but displaying much more stochasticity in the hybrid. Further, among-line variance in ROS level did not follow the expected pattern of increase in this experiment. nad5 $\Delta$ increased linearly with successive generations of inbreeding until reaching a plateau when nad5 $\Delta$ heteroplasmy levels reached $\sim 50 \%$. No relationship between the patterns of ROS and nad5 $\Delta$ evolution were detected. MA lines derived from high-nad5 isolate HK105 went extinct after 20 generations of MA, lending further support for the idea that high nad5 $\Delta$ heteroplasmy is detrimental. These data suggest a threshold level for deletion-bearing genomes within natural populations of $C$. briggsae, above which detrimental phenotypes emerge. In Chapter four I examined the role of ROS and oxidative stress in contributing to heritable nuclear DNA mutation in MA lines of $C$. elegans that had previously been subjected to whole-genome sequencing. We found significant variation in ROS levels among $C$. elegans MA lines, but no variation in 8-oxo-dG levels. Despite the strong relationship between steady-state 8-oxo-dG and ROS, absolutely no correlation between oxidative stress levels and nuclear genome base 
substitution rate was revealed. This leaves open the possibility that ROS may induce other types of mutations or more substantial damage to the mitochondrial genome. Alternatively, ROS may play an indirect role in C. elegans germline mutation processes. In Chapter five, I re-analyzed the data from Chapter two to evaluate patterns of phenotypic correlation for 24 mitochondrial traits within C. briggsae natural isolates, with the aim of identifying traits with the potential to influence mitochondrial stability and integrity. While $\triangle \Psi M$ was significantly correlated to several traits describing mitochondrial shape, number and area, ROS was not related to any of the measured traits. The major patterns of bivariate relationship led to the development of a novel model of mitochondrial population dynamics, in which the overall $\Delta \Psi \mathrm{M}$ influences individual mitochondrial fusion-fission capacities. The model also has broad implications for mitochondrial genome integrity and evolution. Chapter six discusses general conclusions resulting from this work and areas for possible future work. 


\section{CHAPTER TWO}

In vivo quantification reveals extensive natural variation in mitochondrial form and function in Caenorhabditis briggsae

\section{Background}

Mitochondria are organelles that harbor DNA and produce most of the energy (ATP) required to sustain eukaryotic life via an electron transport chain (ETC). Proper assembly and operation of the mitochondrial ETC relies upon the coordinated functioning of both nuclear and mitochondria-encoded subunits of ETC complexes. Hence, mutations affecting ETC genes can have a variety of detrimental consequences that manifest at cellular, tissue, and whole organism levels (Wallace 1999; Schon and Manfredi 2003), and have been implicated in many complex human diseases (Grad et al. 2005; Haas 2010; Winklhofer and Haass 2010; lenco et al. 2011). Proximal effects of ETC mutations include altering mitochondrial reactive oxygen species (ROS) production (Verkaart et al. 2007; Dingley et al. 2009), membrane potential $(\Delta \Psi \mathrm{M})$ (Ventura et al. 2006; Gaskova et al. 2007; Dingley et al. 2009; Lemire et al. 2009), and other aspects of mitochondrial physiology. ROS are generated by the ETC as a byproduct of oxidative phosphorylation and are of particular interest because, when present at high levels, they can damage cellular macromolecules including mitochondrial and nuclear DNA and the components of the ETC itself (Tuma 2001). Because ETC genetic variation can ultimately generate variation in organismal fitness through its effects on physiology, it is likely to be a significant target of natural selection. Accordingly, selection on ETC- 
dependent mitochondrial traits has been implicated in the evolution and diversification of life-history traits (Dowling and Simmons 2009; Monaghan et al. 2009), thermal tolerance (Fangue et al. 2009; Morley et al. 2009), aging (Balaban et al. 2005), reinforcement and allopatric speciation (Ballard and Whitlock 2004; Willett and Burton 2004; Burton et al. 2006, 2007; Gershoni et al. 2009; Keller and Seehausen 2012).

Despite the broad relevance of mitochondria to evolutionary processes and human health, understanding mitochondrial genotype-phenotype relationships has proven difficult (Ballard and Melvin 2010). One reason for this is the variable phenotypic expression of mitochondrial ETC mutations caused by variation in mitochondrial DNA (mtDNA) mutation heteroplasmy (Rossignol et al. 2003; Chen et al. 2010). Heteroplasmy occurs when both wildtype and mutant mtDNA genomes coexist within a mitochondrion, cell, tissue, or individual, and is a common characteristic of mtDNA mutations (Lightowlers et al. 1997). Heteroplasmy is the root cause of mitochondrial threshold effects wherein phenotypic consequences of mtDNA mutations only arise when heteroplasmy level reaches some threshold (Rossignol et al. 2003; Chen et al. 2010). For such reasons, we still have a limited understanding of the causes and consequences of ETC mutations in vivo, and virtually no such information on naturally occurring heteroplasmic ETC mutations. To date, most studies have been conducted on isolated mitochondrial fractions, chemically treated cell lines or yeast strains, or on those containing experimentally generated mutations (Tuppen et al. 2010). 
Advances in biological imaging techniques have facilitated recent in vivo studies of mitochondria and are permitting unprecedented views into the dynamic structure of mitochondria and mitochondrial populations. Regular cycles of fission, fusion, and degradation are now known to maintain organellar populations and contribute to spatiotemporal variation in mitochondrial morphology (Twig et al. 2008a; Graef and Nunnari 2011). Recent studies have identified and characterized genes that control mitochondrial fission and fusion (Labrousse et al. 1999; Chen and Chan 2005; Jagasia et al. 2005; Okamoto and Shaw 2005; Chan 2006a). In addition to these loci, mutations in a number of ETC genes also result in abnormal mitochondrial morphology (Lee et al. 2003; Pham et al. 2004; Duvezin-Caubet et al. 2006). It is increasingly apparent that mitochondrial shape and function are intimately linked such that changes in morphology can affect diverse processes such as free radical signaling, energy metabolism (Dimmer et al. 2002; Lee et al. 2003; Okamoto and Shaw 2005; Chan 2006b; Ichishita et al. 2008), and cell cycle regulation (Mitra et al. 2009). However, the physiological causes of altered mitochondrial shape and the effects of organellar morphology on organellar function are poorly known. Further, we have no information regarding standing levels of variation in these mitochondrial traits or its evolutionary consequences.

Recently, geographically diverse isolates of $C$. briggsae nematodes have been studied with the aim of developing a model for natural population genetic and genomic studies (Cutter et al. 2006; Estes et al. 2011; Ross et al. 2011; Clark et al. 2012). Like C. elegans, C. briggsae is globally distributed and offers many of the same advantages as 
an experimental system (Gupta et al. 2007), however, C. briggsae exhibits higher rates of mutation (Baer et al. 2005; Howe et al. 2010), greater molecular genetic diversity, and more extreme population subdivision than C. elegans. Nuclear (Cutter et al. 2006) and mitochondrial (Howe and Denver 2008) genetic analyses have identified four major phylogeographic clades of $C$. briggsae (the distal three of which are shown in Fig. 2.1A), which may be adapted to local thermal regimes (Jovelin and Cutter 2011; Prasad et al. 2011) and other conditions. Howe and Denver (2008) discovered that many isolates of this species contain varying levels of a large, heteroplasmic mtDNA deletion named nad5 $\Delta$ (Fig. 2.1B and further described in Materials and Methods). The deletion removes nearly half of the $\mathrm{NADH}$-dehydrogenase 5 (nad5) gene (Fig. 2.1B), which encodes a central subunit of mitochondrial ETC complex I. nad5 $\Delta$ appears to occur via illegitimate recombination events between the nad5 gene and an upstream pseudogene, $\Psi$ nad5- 2 , derived from nad5. The "Kenya" (aka Nairobi) C. briggsae clade does not contain the pseudogene and therefore does not experience the deletion, while other $C$. briggsae isolates exhibit nad5 $\Delta$ heteroplasmy levels ranging from zero to over $50 \%$. A number of findings suggest that nad5 $\Delta$ might be detrimental for several aspects of nematode health and fitness - especially when heteroplasmy levels exceed $\sim 40 \%$ (Howe and Denver 2008; Estes et al. 2011) - as expected based on studies of nad5 and other complex I mutants (Shigenaga et al. 1994; Kayser et al. 2004; Biskup and Moore 2006; Janssen et al. 2006; Sedensky and Morgan 2006; Falk et al. 2008). Together with recent work showing that nad5 $\Delta$ behaves as a selfish genetic element; i.e., experiences a strong 


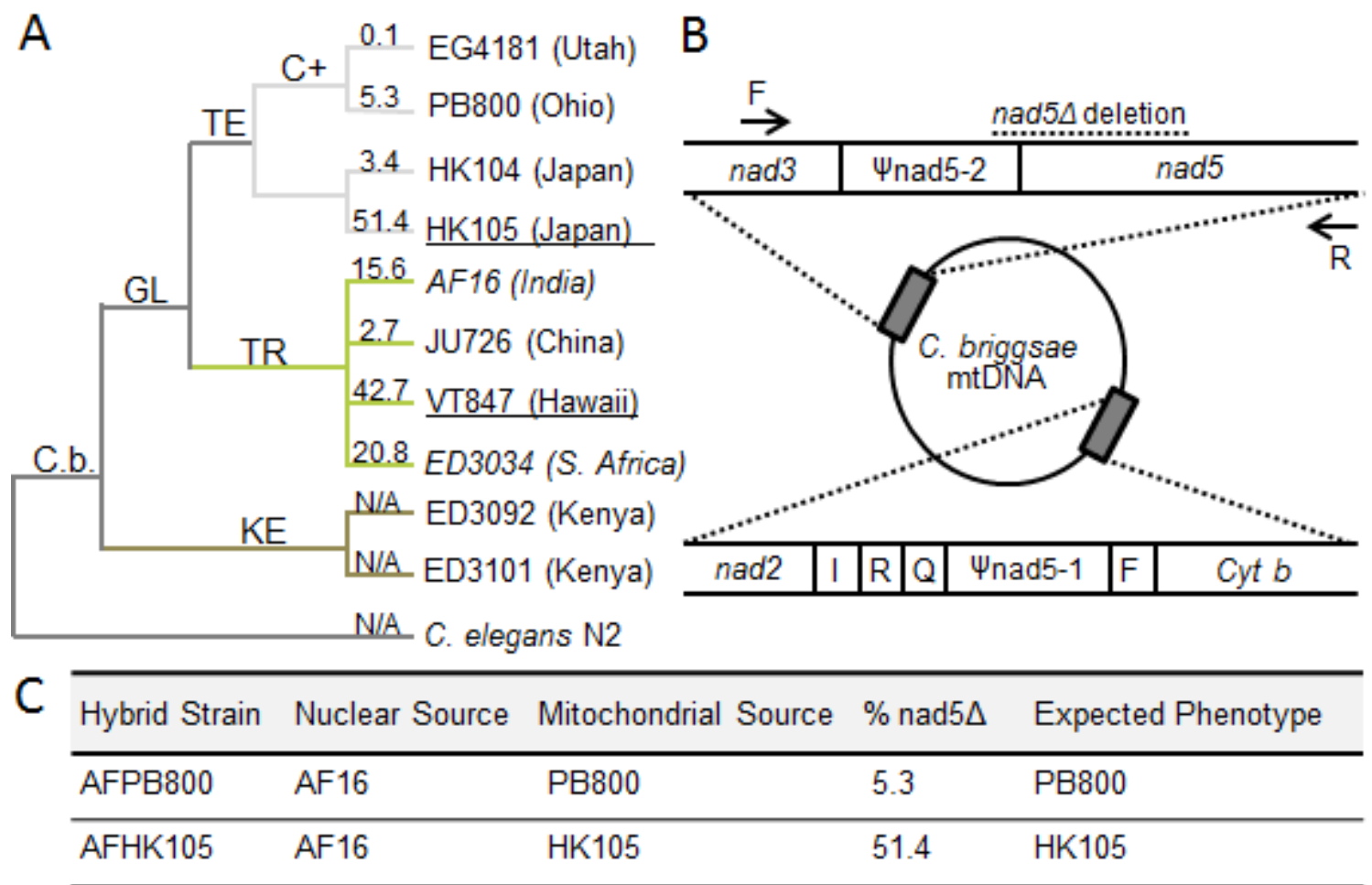

Figure 2.1. Natural and experimental $C$. briggsae strains and description of the nad54 mtDNA deletion. A. Phylogenetic relationship and nad5 $\Delta$ heteroplasmy level of $C$. briggsae isolates studied here. $\mathrm{GL}=$ global superclade; $\mathrm{KE}=$ Kenya clade; TE and TR = temperate and tropical subclades of $\mathrm{GL}$; $\mathrm{C}(+)=$ isolates bearing compensatory $\Psi_{\text {nad } 5 \Delta-2}$ allele; nad $5 \Delta$ heteroplasmy categories were assigned to each C. briggsae natural isolate for statistical analysis following Estes et al. (2011): High = underlined font, medium = italicized, low = regular, and zero-nad5 $=$ "N/A". Note that we assayed the natural HK104 isolate here instead of the mutation-accumulation line progenitor reported in Estes et al. (2011), which had evolved high nad5 $\Delta$ levels in the lab (See Materials and Methods). B. Positions of the nad5 deletion (dashed line at top) and $\Psi_{\text {nad } 5 \Delta-2}$ elements in the mitochondrial genome. Primers are indicated by arrows (adapted from Howe and Denver, 2008). C. Mitochondrial and nuclear parent isolates for each mitochondrial-nuclear hybrid. nad5 5 heteroplasmy for each hybrid strain matches that of the maternal isolates as expected (see methods). Mitochondrial phenotypes are expected to match those of the maternal isolate if measured traits are predominantly determined by the mitochondrial genotype.

transmission bias (Clark et al. 2012), these results indicate that isolate-specific nad5 $\Delta$

levels are probably shaped by a combination of evolutionary forces: recurrent mutation and deletion transmission biases, genetic drift due to sampling of mitochondria during fertilization, and truncation selection.

With the goal of further characterizing global phenotypic variation in $C$. briggsae to include levels of variation in sub-cellular traits, mitochondrial form and 
function was analyzed using natural and experimental hybrid populations of nematodes.

This study provides an assessment of natural levels of physiological and subcellular phenotypic variation and takes a first step toward understanding how this species' extensive ETC genetic variation may affect cellular traits in vivo. We identified traits that diagnose mitochondrial dysfunction and hypothesized that $C$. briggsae isolates with higher nad5 $\Delta$ levels would exhibit mitochondrial phenotypes often associated with such dysfunction, including increased ROS, decreased $\Delta \Psi \mathrm{M}$, and more fragmented mitochondria. However, several mitochondrial phenotypes were non-linearly associated with nad5 5 level and much of the among-isolate phenotypic variation was best explained by phylogeographic clade membership rather than nad5 $\Delta$ frequency. Analysis of mitochondrial-nuclear hybrid strains provided support for both mtDNA and nuclear genetic variation as drivers of natural mitochondrial phenotype variation.

\section{Materials and Methods}

\section{Nematode strains and culture conditions}

For the current study, we used ten natural C. briggsae isolates and two experimental hybrid strains derived from three of these isolates, (Fig. 2.1). The ten natural isolates represent three of the four major phylogeographic clades of $C$. briggsae, encompass the known range of nad5 $\Delta$ heteroplasmy level, and were chosen to include two isolates that do not experience the deletion and two isolates containing a putative compensatory mutation that limits nad5 $\Delta$ formation (Howe and Denver 2008; Estes et 
al. 2011). Note that this study includes a different strain of HK104 from that analyzed in Estes et al. (2011). This study assayed the natural HK104 isolate, which has low nad5 (Fig. 2.1A), while Estes et al. (2011) used an inbred HK104 line that had evolved higher nad5 $\Delta$ frequency. Briefly, nad5 $\Delta$ segregates among natural populations of $C$. briggsae and requires the presence of the $\Psi_{\text {nad } 5 \Delta-2}$ pseudogene (Fig. $2.1 \mathrm{~B}$ ). Sequence repeats within nad5 $\Delta$ and $\Psi_{\text {nad5 }} \Delta-2$ promote direct repeat-associated deletion events. Isolates in the Kenya clade (Fig. 2.1A) lack the pseudogene and are therefore spared nad5 $\Delta$. Among-isolate variation in nad5 $\Delta$ heterolplasmy level is also contolled by the presence of compensatory sequences within the mtDNA of certain isolates As described in (Howe and Denver 2008), the $\Psi_{\text {nad5 }} \Delta-2$ pseudogene directly upstream of $\operatorname{nad} 5 \Delta$ (Fig. 2.1B) is required for the nad5 $\Delta$ deletions to occur within C. briggsae. Directly repeated nucleotide sequences occur within nad5 $\Delta$ and $\Psi_{\text {nad } 5 \Delta-2}$ that promote direct repeat-associated deletion events. Isolates in the "Kenya" clade (Fig. 2.1A) provide natural outgroup controls because they lack $\Psi_{\text {nad }} 5 \Delta-2$ and are thus inherently unable to experience the nad5 $\Delta$ deletions. The among-isolate variability in average heteroplasmy level is thought to also be partly accounted for by the presence of compensatory sequences within the mtDNA of certain isolates (Estes et al. 2011), which appear to place an upper bound on the proportion of nad5-deletion bearing genomes able to accrue within individuals (Howe and Denver 2008). nad5 $\Delta$ was recently shown to behave as a selfish genetic element that increases in frequency when C. briggsae isolates are maintained by single-individual bottlenecking (Clark et al. 2012); 
however, nad5 $\Delta$ heteroplasmy level is relatively stable across generations when isolates are maintained in larger population sizes ( $N$ 100) where natural selection is more efficient (Estes, Coleman-Hulbert, Howe, and Denver, unpubl. data). Great care was taken to ensure that C. briggsae natural isolates did not experience population bottlenecks in the lab and nad5 $\Delta$ heteroplasmy levels were found to remain stable over the course of our study (Estes et al. 2011).

To test the relative contributions of mitochondrial and nuclear genomes to among-isolate phenotypic variability, we also studied two mitonuclear hybrid strains that contain the mitochondrial genome of one isolate (PB800 or HK105) upon the same (AF16) nuclear genetic background (Fig. 2.1C). These strains were generated through serial backcrossing of AF16 males to hermaphrodite cross-progeny for 10 generations. This process is expected to result in worms with $>99.9 \%$ of their nuclear genome from AF16 and their mitochondrial genome from a separate C. briggsae isolate. We confirmed that each hybrid line encoded the expected mtDNA through PCR and direct sequencing of a portion of nad5 (Howe and Denver 2008) and COIl (Denver et al. 2003a) genes. Each hybrid line was also confirmed to harbor nad5 $\Delta$ levels similar to that of the original hermaphrodite mitochondrial donor parent through agarose gel analysis of PCR reactions using primers that flank the deletion region as described in Howe and Denver (2008) (Fig. 2.1C).

Nuclear contributions of hybrid lines were confirmed by evaluating six nuclear PCR loci, one for each of the six C. briggsae chromosomes, that contained single 
nucleotide polymorphism or large-indel variants between the two strains involved in the crosses. SNP variants were evaluated by fluorescent capillary DNA sequencing and large-indel variants were evaluated by agarose gel electrophoresis. The loci used were taken from published variants described in (Koboldt et al. 2010; Raboin et al. 2010). For the AF16 x HK105 hybrid lines (expected to contain AF16 nuclear DNA), 6/6 target loci contained differences between the strains and $6 / 6$ of the loci were confirmed to contain only the AF16 sequence in the hybrid line. For the AF16 x PB800 cross (also expected to contain AF16 nuclear DNA), only 5/6 loci had diagnostic sequence differences between the two strains; the chromosome $\mathrm{V}$ locus employed was identical between AF16 and PB800. However, 5/5 of the diagnostic nuclear loci confirmed the presence of the AF16 sequence only in the hybrid strain.

We note here that we attempted to generate additional sets of mitonuclear hybrid strains by crossing other pairs of $C$. briggsae natural isolates; however, molecular genetic assays revealed that bi-parental inheritance of mitochondrial genotypes had occurred in these lines. In a similar study involving inter-strain crosses in C. elegans, mitochondrial DNA was observed to be strictly maternally inherited (W.K. Thomas and K. Morris, unpubl. data). Thus, our result may suggest that favorable mitonuclear epistatic interactions specific to individual C. briggsae isolates were disrupted in some hybrid strains such that paternal mitochondria were transmitted to hybrid offspring (Zouros 1994; Ellison and Burton 2006; Fontaine et al. 2007; Wolff et al. 2008). Such a situation might arise if hybridization in some way disrupts the autophagic degradation of 
paternal mitochondria that normally occurs after fertilization (Sato and Sato 2011; Zhou et al. 2011), for example. These strains were therefore omitted from the current analysis.

All nematodes were grown under standard laboratory conditions at $25^{\circ} \mathrm{C}$ on 15 mm NGM petri plates seeded with HB101 Escherichia coli. All strains were included in each analysis. Prior to each assay, age synchronous worms were obtained through a standard bleaching protocol.

\section{Fluorescence microscopy}

Confocal image analysis was performed on young adult nematodes treated with mitochondria-targeted fluorescent dyes. Fluorescent imaging provides the distinct advantage of allowing simultaneous localization and relative quantification of mitochondrial traits (Fig. 2.2). Additionally, recent work indicates that ROS data obtained from fluorescent analysis and electron spin resonance yield similar results (Kuznetsov et al. 2011). This dye-based method has received criticism for its potential to be influenced by variable feeding rates (Lee et al. 2010). Importantly, we find no correlation between pharyngeal pumping rates (Estes et al. 2011) and maximal ROS or $\Delta \Psi \mathrm{M}$ as measured here (ROS $\rho=-0.024, \mathrm{p}=0.955 ; \Delta \Psi \mathrm{M} \rho=0.167, \mathrm{p}=0.693)$. Also, unlike dye-based methods performed using whole-worm lysate (B. Halliwell, pers. comm.), our method does not cause the disruption and release of organellar and intracellular contents, which can lead to increased ROS via the release of free iron and ensuing Fenton reactions (Halliwell and Gutteridge 2007). Prior to each assay, age 


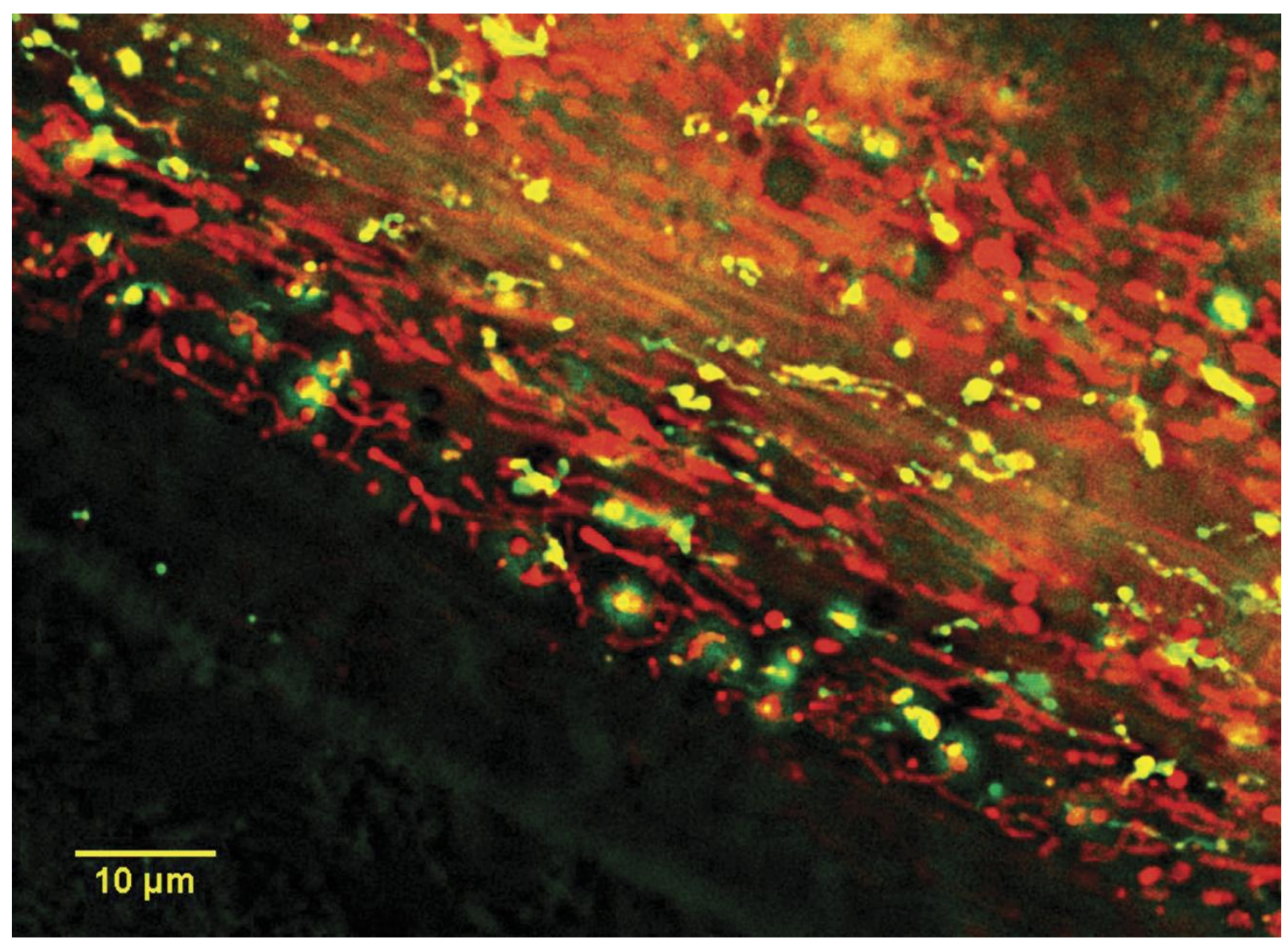

Figure 2.2. Localization of mitochondria-targeted dyes in C. briggsae nematodes. A Z-projection image of MitoTracker Red CMXRos (red objects) and MitoTracker Green FM (green objects) specific staining, and colocalization of these probes (yellow objects) within an individual (after processing to find edges of objects). Green objects are relatively depolarized mitochondria that have taken up primarily MitoTracker Green FM probe; red objects are more polarized mitochondria that have taken up mostly MitoTracker Red probe. Yellow objects likely have intermediate polarization and have taken up equivalent amounts of each probe. Scale bar $=10 \mu \mathrm{m}$.

synchronous worms were incubated with $E$. coli labeled with $10 \mu \mathrm{M}$ concentrations of the specific fluorescent dye(s) appropriate for each experiment. Concurrently, a second age synchronous batch of worms from the same strain was incubated without dye to serve as the control. After 24 hours of exposure, young adult stage worms were washed and transferred to fresh NGM plates containing unlabeled E.coli. Worms were allowed to feed for one hour, which clears the digestive tract of any labeled $E$. coli that could interfere with accurate fluorescence measurements. Immediately prior to imaging, 
worms were paralyzed following the methods of Dingley et al. (2009) with a single drop of $5 \mathrm{M}$ levamisole (Vector Technologies, Inc., Burlingame, CA), which immobilizes worms by preventing depolarization of skeletal muscle (Lewis et al. 1980).

For each dye-based assay, images of the pharynx of each nematode were collected. The pharynx is a neuromuscular organ that nematodes use to ingest bacteria (Albertson and Thomson 1976). We chose to analyze pharyngeal bulbs for two reasons: 1) they are easily visualized and have high levels of dye uptake, which serves to reduce technical variation, and 2) they are mitochondria-rich but contain few lipid droplets, which are highly autofluorescent and thus interfere with accurate measurement of mitochondrial fluorescence. Images were acquired using a high resolution wide field Core DV system (Applied Precision ${ }^{\mathrm{TM}}$, Issaquah, WA) equipped with an Olympus IX71 inverted microscope mounted with a Nikon Coolsnap ES2 HQ camera (Advanced Light Microscopy Core Facility, Oregon Health and Science University, Portland, OR). Fluorescent z-stack images of the pharyngeal bulb of individual worms were captured at 60X magnification using a short arc 250W Xenon lamp. All images were captured immediately following paralysis. Images were optimized by deconvolving, and relative fluorescence values were obtained using ImageJ software (NIH, Bethesda, MD).

Relative mitochondrial oxidant levels

ROS levels were assessed in vivo for all $C$. briggsae isolates following the basic approach of (Dingley et al. 2009) and described further in Estes et al. (2011). Briefly, age-synchronized worms were incubated for 24 hours in 10 uM MitoSOX Red (Molecular 
Probes; Grand Island, NY) before imaging as outlined above. MitoSOX Red was recently shown to quantify total levels of mitochondrial oxidants, rather than superoxide specifically, when used with confocal microscopy (Zielonka and Kalyanaraman 2010). Images were acquired using a high resolution wide field Core DV system (Applied Precision $^{\mathrm{TM}}$ ), equipped with an Olympus IX71 inverted microscope mounted with a Nikon Coolsnap ES2 HQ camera (Advanced Light Microscopy Core Facility, Oregon Health and Science University, Portland, OR). Fluorescent, z-stack images of the mitochondrial-rich pharynx with a 1.0 second exposure time were captured at 60X magnification. Images were deconvolved prior to analysis. Terminal pharyngeal bulbs were manually circled to quantify maximum fluorescence intensity of the area in exposed and unexposed (control) animals for each strain using ImageJ software (NIH). The final pharyngeal bulb intensity values were calculated as the difference between intensity values for exposed and control worms.

The efficacy of a second mitochondrial ROS detecting dye, MitoTracker Red CM$\mathrm{H}_{2} \mathrm{XRos}$ (Molecular Probes Inc., Eugene, OR), was assessed using the same protocol as above. We initially believed that this dye would be preferable over MitoSOX Red because of its greater sensitivity to ROS (Kuznetsov et al. 2011). However, although the fluorescent signal from MitoTracker Red $\mathrm{CM}-\mathrm{H}_{2}$ XRos-treated mitochondria was distinct, the background fluorescence generated from non-specific lipid uptake of the dye decreased the precision of the fluorescence measurement (Waters 2009). MitoSOX Red 
generated less background fluorescence and produced more precise images, and was therefore used for mitochondrial ROS detection.

Although the use of mitochondria-specific dyes has become a widely used and accepted method for measuring a variety of mitochondrial traits in vivo, a caveat is that differential dye uptake between samples could lead to inaccurate interpretation of fluorescence differences. There is currently no means of determining the extent to which this occurs (Dingley et al. 2009). However, great care was taken to minimize all other sources of error. For example, we exposed only one sample at a time to the microscope light source and used the shortest exposure time possible to avoid introducing variation due to breakdown of the dye.

\section{$\underline{\text { Relative mitochondrial } \triangle \Psi \mathrm{M}}$}

Relative $\triangle \Psi \mathrm{M}$ was assessed using MitoTracker Red CMXRos, the uptake of which is dependent upon mitochondrial $\triangle \Psi \mathrm{M}$. We utilized the ROS assessment protocol (above), except that a shorter, 0.08 seconds, exposure time was used for imaging; these assays were performed concurrently with those for mitochondrial morphology (below).

\section{Mitochondrial morphology}

Quantification of differences in mitochondrial morphology was achieved by colabeling worms with MitoTracker Green FM, which is not $\Delta \Psi \mathrm{M}$-specific, and MitoTracker Red CMXRos, which accumulates exclusively within actively respiring mitochondria (Fig. 2.2). This allowed us to distinguish between polarized and depolarized mitochondria. 
Worms were prepared for imaging as above, this time with exposures of 0.08 seconds for MitoTracker Red and 0.02 seconds for MitoTracker Green.

We also confirmed that altered $\Delta \Psi \mathrm{M}$ would produce the expected effects on mitochondrial morphology (Okamoto and Shaw 2005) by depleting $\triangle \Psi \mathrm{M}$ with $10 \mu \mathrm{M}$ carbonylcyanide-3-chlorophenylhydrazone (CCCP, Sigma-Aldrich, St. Louis, MO) in 15 individuals from mid-deletion isolate, ED3034 (Fig. 2.1A). Beginning at the L1 stage, worms were transferred daily to fresh CCCP-treated plates until they reached young adulthood. Compared to an equal number of untreated ED3034 individuals, we found that CCCP treatment reduced the size of both the total and the functional (polarized) mitochondrial population $\left(A_{T p}: F=6.185, p=0.0202, A_{F p}: F=7.607, p=0.0134\right)$, the size of individual functional mitochondria $\left(A_{F}: F=4.591, p=0.0425\right)$, and the variance in circularity of functional mitochondria $\left(\mathrm{C}_{\mathrm{FV}}: \mathrm{F}=7.136, \mathrm{p}=0.0134\right)-$ as expected if organelles are becoming increasingly fragmented. (See below and Table 2.1 for further explanation.)

Mitochondrial localization of fluorescent dyes and effect of levamisole

We observed extensive localization of MitoTracker Green FM and Red CMXRos (Fig. 2.2), which points to mitochondria-specific staining by fluorescent dyes. This specificity was further confirmed by depleting $\triangle \Psi M$ using CCCP (as above) and directly visualizing reductions in the fluorescence intensity of all probes used, especially the membrane-potential dependent dyes (data not shown). We also tested the effect of levamisole, the drug used to paralyze worms prior to imaging, on dye fluorescence using 


\begin{tabular}{|c|c|c|c|c|}
\hline \multirow{2}{*}{ Label } & \multirow{2}{*}{ Trait } & \multirow{2}{*}{ Description } & \multirow{2}{*}{$\begin{array}{l}\text { Grand } \\
\text { mean }\end{array}$} & \multirow{2}{*}{$\frac{F}{d f}$} \\
\hline & & & & \\
\hline \multicolumn{5}{|c|}{ Measures of Organismal Physiology } \\
\hline \multirow{2}{*}{ ATP } & \multirow{2}{*}{ Total ATP content } & \multirow{2}{*}{$\begin{array}{l}\text { Steady-state ATP levels normalized by } \\
\text { protein content (nM/mg protein) }\end{array}$} & \multirow{2}{*}{4.499} & $25.09 * * *$ \\
\hline & & & & 84,8 \\
\hline \multirow{2}{*}{ PR } & \multirow{2}{*}{$\begin{array}{l}\text { Paraquat } \\
\text { Resistance }\end{array}$} & \multirow{2}{*}{$\begin{array}{l}\text { Total time alive in minutes in } 300 \mathrm{mM} \\
\text { paraquat }\end{array}$} & \multirow{2}{*}{61.03} & $7.139 * * *$ \\
\hline & & & & 126,8 \\
\hline \multicolumn{5}{|c|}{ Measures of Mitochondrial Physiology } \\
\hline \multirow{2}{*}{$\begin{array}{l}\triangle \Psi \mathrm{M} \\
\text { Mean }\end{array}$} & \multirow{4}{*}{$\begin{array}{l}\text { Membrane } \\
\text { potential }\end{array}$} & \multirow{2}{*}{$\begin{array}{l}\text { Average of mean relative MitoTracker } \\
\text { Red CMXRos fluorescence }\end{array}$} & \multirow{2}{*}{836.8} & $90.99 * * *$ \\
\hline & & & & 182,9 \\
\hline \multirow{2}{*}{$\begin{array}{l}\Delta \Psi \mathrm{M} \\
\mathrm{Max}\end{array}$} & & \multirow{2}{*}{$\begin{array}{l}\text { Average of max relative MitoTracker } \\
\text { Red CMXRos fluorescence }\end{array}$} & \multirow{2}{*}{2331} & $75.20 * * *$ \\
\hline & & & & 182,9 \\
\hline \multirow{2}{*}{$\begin{array}{l}\text { ROS } \\
\text { Mean }\end{array}$} & \multirow{4}{*}{$\begin{array}{l}\text { Reactive Oxygen } \\
\text { Species }\end{array}$} & \multirow{2}{*}{$\begin{array}{l}\text { Average of mean relative MitoSOX } \\
\text { Red fluorescence }\end{array}$} & \multirow{2}{*}{298} & $9.269 * * *$ \\
\hline & & & & 201,9 \\
\hline \multirow{2}{*}{$\begin{array}{l}\text { ROS } \\
\text { Max }\end{array}$} & & \multirow{2}{*}{$\begin{array}{l}\text { Average of max relative MitoSOX Red } \\
\text { fluorescence }\end{array}$} & \multirow{2}{*}{1643} & $12.18 * * *$ \\
\hline & & & & 201,9 \\
\hline \multicolumn{5}{|c|}{ Measures of the Mitochondrial Population } \\
\hline \multirow{2}{*}{$A_{F P}$} & & & 2870 & $4.923 * * *$ \\
\hline & & & 200 & 169,9 \\
\hline & Area of & Area of functional, non-functional or & & $2.012^{*}$ \\
\hline$A_{N P}$ & $\begin{array}{l}\text { mitochondrial } \\
\text { population }\end{array}$ & $\begin{array}{l}\text { total (both functional and non- } \\
\text { functional) mitochondrial populations }\end{array}$ & 3152 & 169,9 \\
\hline$A_{-P}$ & & & ברח6 & $3.349 * * *$ \\
\hline$A_{T P}$ & & & bULZ & 169,9 \\
\hline$A_{-}$ & Ratio of functional & Area of the functional mitochondrial & 1151 & $3.703 * * *$ \\
\hline AFP/NP & mitochondrial area & population & 1.101 & 169,9 \\
\hline & & Area of the functional mitochondrial & & $3.434 * * *$ \\
\hline$A_{F P / T P}$ & $\%$ functional area & $\begin{array}{l}\text { population/area of the total } \\
\text { population }\end{array}$ & 0.443 & 169,9 \\
\hline $\mathrm{N}_{\mathrm{c}}$ & & & 67.91 & $6.247^{* * *}$ \\
\hline$\omega_{F}$ & Number of & Number of functional, non-functional, & & 169,9 \\
\hline$N_{1}$ & mitochondria & or total individual mitochondria & 5885 & $2.143^{*}$ \\
\hline $\mathbf{N}_{\mathrm{N}}$ & & & 30.80 & 169,9 \\
\hline
\end{tabular}




\begin{tabular}{|c|c|c|c|c|}
\hline \multirow{2}{*}{$\mathrm{N}_{\mathrm{T}}$} & & & \multirow{2}{*}{124.7} & $3.977^{* * *}$ \\
\hline & & & & 169,9 \\
\hline \multirow[b]{2}{*}{$\mathbf{N}_{\mathrm{F} / \mathrm{N}}$} & \multirow{2}{*}{$\begin{array}{l}\text { Ratio of functional } \\
\text { to non-functional } \\
\text { mitochondria }\end{array}$} & \multirow{2}{*}{$\begin{array}{l}\text { Number of functional } \\
\text { mitochondria/number of non- } \\
\text { functional mitochondria }\end{array}$} & \multirow[b]{2}{*}{1.331} & $3.451 * * *$ \\
\hline & & & & 169,9 \\
\hline \multirow{2}{*}{$\mathrm{N}_{\mathrm{F} / \mathrm{T}}$} & \multirow{2}{*}{$\begin{array}{l}\% \text { functional } \\
\text { mitochondria }\end{array}$} & \multirow{2}{*}{$\begin{array}{l}\text { Number of functional } \\
\text { mitochondria/number of total } \\
\text { mitochondria }\end{array}$} & \multirow{2}{*}{0.505} & $3.983 * * *$ \\
\hline & & & & 169,9 \\
\hline \multicolumn{5}{|c|}{ Measures of Individual Mitochondrial Shape } \\
\hline \multirow{2}{*}{$A_{F}$} & \multirow{4}{*}{$\begin{array}{l}\text { Area of individual } \\
\text { mitochondria }\end{array}$} & \multirow{4}{*}{$\begin{array}{l}\text { Average area of individual functional } \\
\text { or non-functional mitochondria }\end{array}$} & \multirow{2}{*}{40.74} & 1.504 \\
\hline & & & & 169,9 \\
\hline \multirow{2}{*}{$A_{N}$} & & & \multirow{2}{*}{55.7} & $2.284^{*}$ \\
\hline & & & & 169,9 \\
\hline \multirow{2}{*}{$\mathrm{AR}_{\mathrm{F}}$} & \multirow{4}{*}{ Aspect ratio } & \multirow{4}{*}{$\begin{array}{l}\text { Average of the ratio between the } \\
\text { major and minor axis of the ellipse } \\
\text { equivalent to each functional or non- } \\
\text { functional mitochondrion }\end{array}$} & \multirow{2}{*}{1.669} & 1.499 \\
\hline & & & & 169,9 \\
\hline \multirow{2}{*}{$A R_{N}$} & & & \multirow{2}{*}{1.865} & $5.232 * * *$ \\
\hline & & & & 169,9 \\
\hline \multirow{2}{*}{$\mathrm{AR}_{\mathrm{FV}}$} & \multirow{4}{*}{$\begin{array}{l}\text { Aspect ratio } \\
\text { variance }\end{array}$} & \multirow{4}{*}{$\begin{array}{l}\text { Average within-individual variance in } \\
\text { aspect ratio of functional or non- } \\
\text { functional mitochondria }\end{array}$} & \multirow{2}{*}{0.655} & 1.875 \\
\hline & & & & 163,9 \\
\hline \multirow{2}{*}{$\mathrm{AR}_{\mathrm{NV}}$} & & & \multirow{2}{*}{1.271} & 1.348 \\
\hline & & & & 166,9 \\
\hline \multirow{2}{*}{$\mathrm{C}_{\mathrm{F}}$} & \multirow{4}{*}{ Circularity } & \multirow{4}{*}{$\begin{array}{l}4 \pi \text { (area/perimeter }{ }^{2} \text { ) for functional or } \\
\text { non-functional mitochondria }\end{array}$} & \multirow{2}{*}{0.8619} & 1.771 \\
\hline & & & & 169,9 \\
\hline$C_{u}$ & & & 0825 & $3.494 * * *$ \\
\hline $\mathbf{C N}_{\mathbf{N}}$ & & & 0.020 & 169,9 \\
\hline & & & & 1.891 \\
\hline$C_{F V}$ & Circularity pariance & Within-individual variance in & $0.03 /$ & 163,9 \\
\hline$C_{N}$ & Circuiaricy variatice & functional mitochondria & 0.052 & $2.049 *$ \\
\hline$C_{N V}$ & & & 0.032 & 166,9 \\
\hline
\end{tabular}

Table 2.1 Assigned labels and descriptions of all mitochondrial traits measured for $C$. briggsae natural isolates. The grand mean, F-ratio and degrees of freedom for one-way ANOVA testing for phenotypic differences among $C$. briggsae isolates. Bold font identifies the nine traits retained in the classification tree analysis when using categories based on isolate-specific nad5 $\Delta \%$ (see Table 2.3). *, $* *$, and ${ }^{* * *}$ denote $\mathrm{p}<0.05,0.01,0.001$, respectively. Subscripts $\mathrm{N}, \mathrm{F}$, and $\mathrm{T}$ indicate whether the measure refers to Non-functional, Functional, or Total mitochondria. Subscript $\mathrm{P}$ and $\mathrm{V}$ denote that 
the measure refers to the entire mitochondrial population (not individual mitochondria), or the average individual variance in that trait, respectively.

five age synchronous individuals from isolates PB800 and HK105 (Fig. 2.1A). There was no significant effect of levamisole on the mean or maximum fluorescence values of MitoTracker Red CMXRos or MitoTracker Green FM, nor was there a levamisole-byisolate interaction. Similarly, levamisole had no effect on the maximum values of MitoSOX fluorescence (t-tests, $p>0.291$ ); however, there were significant effects of levamisole $(t=-2.29, p=0.038)$ and the interaction of levamisole and isolate $(t=-2.13, p$ $=0.051$ ) on mean MitoSOX fluorescence. Maximum fluorescence values were therefore used for all statistical analyses so that any isolate-by-probe interactions generated by levamisole were unlikely to influence our among-isolate comparisons. Furthermore, maximum measures are more consistent in fluorescence image analysis because they are unaffected by variation in pixel size and mitochondrial number or area between images.

We also attempted to co-label nematodes treated as above with either DAPI or Hoechst 33342 (Sigma-Aldrich, St. Louis, MO) in order to visualize cell nuclei, which would have allowed us to assess the intracellular distribution of mitochondria. (Appropriate GFP fusions are not yet available for $C$. briggsae.) Unfortunately, both DAPI and Hoechst noticeably interfered with the fluorescence of the above MitoTracker dyes in C. briggsae (Hicks, pers. obs.). Our study therefore focuses on properties of individual mitochondria and mitochondrial populations within the pharyngeal tissue. $\underline{\text { Image analysis }}$ 
All image analyses were performed in ImageJ (NIH, ver. 1.43u). Quantification of relative ROS and $\triangle \Psi M$ levels was achieved by manually enclosing the terminal pharyngeal bulb of each image to find the average intensity of the area as described in (Dingley et al. 2009). Mitochondrial morphology traits were quantified by processing images following (Koopman et al. 2006). Briefly, visibility of MitoTracker stained structures was improved by applying a linear stretch of the pixel intensity histogram corresponding to each slice in the $z$ stack. This process enhances the contrast of an image by adjusting the number of low and high intensity pixels in the image based on the lowest and highest pixel values in the current image (Russ 2002). The image was then converted into a z-projection, a process that effectively removes the spaces between each slice of the $z$-stack creating a composite image from all slices. A 7x7 tophat filter was then applied, followed by a median filter and a thresholding step (Koopman et al. 2006). The thresholded image was then converted to a binary image, which results in white mitochondria on a black background that can be analyzed in ImageJ.

To quantify among-isolate differences in mitochondrial form and function, we defined and measured 24 traits (Table 2.1). We evaluated functionality of mitochondria based on relative membrane potential ( $\triangle \Psi M$ mean, $\triangle \Psi M$ max) and reactive oxygen species (ROS mean, ROS max). We also quantified various features of the mitochondrial population: the combined area of the mitochondrial population $\left(A_{F P}, A_{N P}, A_{T P}\right)$, the ratio of the area of functional to non-functional mitochondria $\left(A_{F P / N P}\right)$, and the percentage of 
the total mitochondrial area that is functional $\left(A_{F P / T P}\right)$, the number of organelles $\left(N_{F}, N_{N}\right.$, $\mathrm{N}_{\mathrm{T}}$ ), the ratio of functional to non-functional organelles $\left(\mathrm{N}_{\mathrm{F} / \mathrm{N}}\right)$, and the percentage of functional mitochondria $\left(\mathrm{N}_{\mathrm{F} / \mathrm{T}}\right)$, as defined by uptake of MitoTracker Red CMXRos. To describe organellar shape differences we quantified the area $\left(A_{F}, A_{N}\right)$, aspect ratio $\left(A R_{F}\right.$, $\left.A R_{N}\right)$, and circularity $\left(C_{F}, C_{N}\right)$ of individual mitochondria. Aspect ratio measures the ratio between the long and short axes of an ellipse fit to the object in question (Russ 2002). It has a minimal value of 1 , which corresponds to a perfect circle. Circularity (sometimes referred to as formfactor in the literature) is calculated as $4 \Pi\left(\right.$ area/perimeter ${ }^{2}$ ), and will also equal 1 when the measured object is a perfect circle. As the object becomes more elongated and/or branched, circularity approaches 0 (Russ 2002). Because circularity cannot accurately be measured for extremely small objects (ImageJ website), we omitted from all analyses mitochondria smaller than 2 pixels (or 0.129 microns). The number of such objects removed from analyses was small (ranging from 0-20 with most $<10)$ and did not differ among isolates $(F<1.618, p>0.094)$; this procedure likely had no impact on our phenotypic comparisons among isolates. Finally, to examine heterogeneity in mitochondrial form we estimated within-individual variance in aspect ratio $\left(A R_{F V}, A R_{N V}\right)$ and circularity $\left(C_{F V}, C_{N V}\right)$ of mitochondria for all strains.

\section{$\underline{\text { Statistical analysis }}$}

Classification trees were used to determine which mitochondrial characteristics most accurately grouped $C$. briggsae isolates into categories corresponding to nad5 $\Delta$ heteroplasmy level or to phylogeographic clades. A classification or decision tree is a 
data reduction technique that predicts the membership of data points within classes of a categorical "dependent" variable (Gotelli and Ellison 2004). We chose the classification tree method over PCA or discriminant analysis because the latter techniques assume linearity and equal variance among groups, which our data violated. We first performed one-way analyses of variance (ANOVA) for each of the 24 mitochondrial traits to determine which of them varied significantly among the natural isolates. (Nonparametric analyses gave the same results and are not presented.) Eighteen of 24 traits exhibited significant among-isolate variation (Table 2.1) and were retained as descriptor variables in the classification tree analyses. For the first analysis, the ten natural isolates were grouped into four categories corresponding to their relative levels of nad5 $\Delta$ frequency as before (Estes et al. 2011). Classification trees were run five times for both analyses. For each run, traits that were retained in the tree were recorded along with the misclassification rate and $R^{2}$ value. We considered any trait retained in 4 of 5 runs to be important under that scheme.

Analysis of among-isolate or hybrid strain variation was performed using separate one-way analyses of variance (ANOVA) for each phenotype measured (above). Least-squares contrasts (Tukey's HSD for all pairwise comparisons) were used to test for differences between pairs of isolates. Additionally, we tested the effect of phylogenetic clade and assessed within-clade variation using nested ANOVA for each trait. To test for associations between traits and nad5 $\Delta$ levels in $C$. briggsae, each trait was regressed 
onto isolate-specific nad5 percentages. All analyses were performed in JMP 9 (SAS Institute, Cary, NC).

\section{Results}

\section{Natural variation in mitochondrial phenotypes}

We quantified natural variation in 24 phenotypes that describe mitochondrial function and shape (Table 2.1) among ten distinct C. briggsae isolates (Fig. 2.1A). A majority (18 of 24) of the measured traits exhibited significant among-isolate variation. Mitochondrial $\triangle \Psi M$ showed the greatest among-isolate diversity, followed by mitochondrial ROS levels (Table 2.1; Fig. 2.3). Notably, mitochondria that we considered to be functional (i.e., polarized) by virtue of their having sufficient $\Delta \Psi M$ to permit uptake of MitoTracker Red CMXRos (Fig. 2.2; Materials and Methods) did not vary significantly among isolates in shape or heterogeneity in shape (Table 2.1). In contrast, characteristics of shape and heterogeneity in non-functional (depolarized) mitochondria often differed significantly among isolates. Traits that describe features of mitochondrial populations (i.e., all ten traits that describe the combined area or number of mitochondria), however, differed among isolates for both functional and non-functional mitochondria (Table 2.1).

Striking variation was found among phylogeographic clades of $C$. briggsae (Fig. 2.3; Table 2.2). Of the nine traits that best distinguished isolates with different nad5 $\Delta$ 

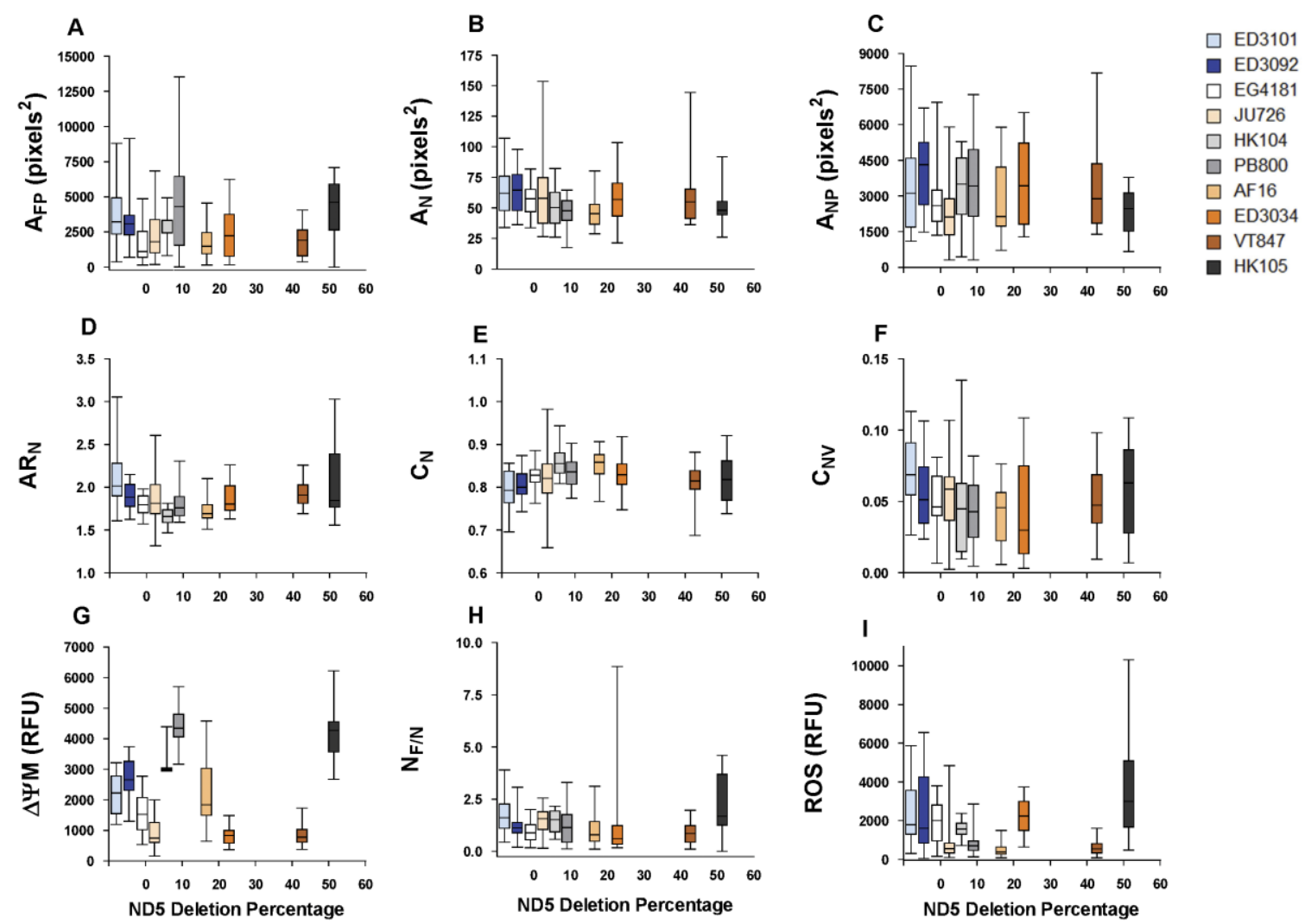

Figure 2.3. Associations between mitochondrial function and morphology traits and isolatespecific nad5 $\Delta$ level. Natural variation among $C$. briggsae isolates in (A) the total area of functional mitochondria, (B) the average area of individual non-functional mitochondria, (C) the total area of non-functional mitochondria, the (D) aspect ratio, (E) circularity, (F) circularity variance of non-functional mitochondria, in (G) relative $\triangle \Psi \mathrm{M}$, (I) the ratio of functional to nonfunctional organelles, and $(\mathrm{H})$ relative ROS levels. Column colors corresponding to phylogenetic clade (orange $=$ Kenya, white $=$ Temperate, blue $=$ Tropical), and isolates are ordered by deletion frequency along the $x$-axis. ED3101 and ED3092 do not experience the deletion and were assigned arbitrary $x$-values of -7 and -5 , respectively, for this figure. Averages of maximum pharyngeal bulb fluorescence in $C$. briggsae natural isolates are plotted in relative fluorescence units (RFU). Bars represent one SEM for 15-20 independent samples.

levels (see below), all but $\mathrm{N}_{\mathrm{F} / \mathrm{N}}$ - the ratio of the number of functional to non-functional organelles - varied significantly among C. briggsae clades, consistent with a phylogenetic effect on many mitochondrial traits. In all of these cases, among-clade variation exceeded the variation observed within clades (Table 2.2). To further explore among-clade phenotypic differences, we used classification tree analysis to identify 


\begin{tabular}{|c|c|c|c|}
\hline Label & Trait & Source & $\mathbf{F}$ \\
\hline \multirow{2}{*}{$A_{N}$} & \multirow{2}{*}{$\begin{array}{l}\text { Area of individual non- } \\
\text { functional mitochondria }\end{array}$} & Clade & $4.593^{* *}$ \\
\hline & & Isolate(Clade) & 1.706 \\
\hline \multirow{2}{*}{$A_{N P}$} & \multirow{2}{*}{$\begin{array}{l}\text { Area of the non- } \\
\text { functional mitochondrial } \\
\text { population }\end{array}$} & Clade & $3.365^{*}$ \\
\hline & & Isolate(Clade) & 1.624 \\
\hline \multirow{2}{*}{$A_{F P}$} & \multirow{2}{*}{$\begin{array}{l}\text { Area of the functional } \\
\text { population }\end{array}$} & Clade & $8.678 * * *$ \\
\hline & & Isolate(Clade) & $3.639 * * *$ \\
\hline \multirow{2}{*}{$A R_{N}$} & \multirow{2}{*}{$\begin{array}{l}\text { Aspect ratio of non- } \\
\text { functional mitochondria }\end{array}$} & Clade & $7.135^{* * *}$ \\
\hline & & Isolate(Clade) & $4.644 * * *$ \\
\hline \multirow{2}{*}{$C_{N}$} & \multirow{2}{*}{$\begin{array}{l}\text { Circularity of individual } \\
\text { non-functional } \\
\text { mitochondria }\end{array}$} & Clade & $8.125^{* * *}$ \\
\hline & & Isolate(Clade) & $2.218^{*}$ \\
\hline \multirow{2}{*}{$C_{N V}$} & \multirow{2}{*}{$\begin{array}{l}\text { Variance in circularity of } \\
\text { non-functional } \\
\text { mitochondria }\end{array}$} & Clade & $3.574 *$ \\
\hline & & Isolate(Clade) & 1.549 \\
\hline \multirow{2}{*}{$\begin{array}{l}\triangle \Psi \mathrm{M} \\
\operatorname{Max}\end{array}$} & \multirow{2}{*}{$\begin{array}{l}\text { Mitochondrial membrane } \\
\text { potential }\end{array}$} & Clade & $187.4^{* * *}$ \\
\hline & & Isolate(Clade) & $43.02 * * *$ \\
\hline \multirow{2}{*}{$\mathrm{N}_{\mathrm{F} / \mathrm{N}}$} & \multirow{2}{*}{$\begin{array}{l}\text { The ratio of functional to } \\
\text { non-functional } \\
\text { mitochondria }\end{array}$} & Clade & 2.226 \\
\hline & & Isolate(Clade) & $3.748 * * *$ \\
\hline \multirow{2}{*}{$\begin{array}{l}\text { ROS } \\
\text { Max }\end{array}$} & \multirow{2}{*}{$\begin{array}{l}\text { Reactive Oxygen Species } \\
\text { (Average of max relative } \\
\text { MitoSOX Red } \\
\text { fluorescence) }\end{array}$} & Clade & $18.48^{* * *}$ \\
\hline & & Isolate(Clade) & $10.21 * * *$ \\
\hline
\end{tabular}

Table 2.2. Effect of phylogenetic clade $(\mathrm{df}=2$ ) and strain nested within clade ( $d f=7$ ) for mitochondrial form and function traits. The F-ratio and degrees of freedom for nested analyses of variance for each trait are shown with $*, * *$, and $* * *$ denoting $\mathrm{p}<0.05,0.01,0.001$, respectively. Subscripts $\mathrm{N}$ and $\mathrm{F}$ indicate whether the measure refers to Non-functional or Functional mitochondria. Subscript $P$ denotes that the measure refers to the entire mitochondrial population, rather than individual mitochondria. Subscript $\mathrm{V}$ denotes measures of average individual variance in that trait. 
which of the 24 measured mitochondrial traits best distinguished three major phylogeographic clades of $C$. briggsae (Table 2.3). Classification trees invariably retained only $\triangle \Psi \mathrm{M}$ in the analysis. Misclassification rates were somewhat high (34\%); isolatespecific $\triangle \Psi M$ nonetheless accounted for $20 \%$ of the total variation among clades. Further, if we allowed classification trees to continue splitting beyond the optimal number of splits (i.e., when further partitioning failed to account for a significant fraction of the total variation), analyses again used $\triangle \Psi \mathrm{M}$ to further classify groups of isolates.

\begin{tabular}{ll}
\hline $\begin{array}{l}\text { Misclassification } \\
\text { rate }\end{array}$ & 0.3427 \\
$\mathrm{R}^{2}$ & 0.2055 \\
Traits Used & $\Delta \Psi \mathrm{M}$ \\
\hline Misclassification & 0.3305 \\
rate & 0.4518 \\
$\mathrm{R}^{2}$ & $\mathrm{ROS}, \Delta \Psi \mathrm{M}, \mathrm{A}_{\mathrm{N}}, \mathrm{A}_{\mathrm{NP}}, \mathrm{A}_{\mathrm{FP}}, \mathrm{AR}_{\mathrm{N}}, \mathrm{C}_{\mathrm{N}}, \mathrm{C}_{\mathrm{NV}}, \mathrm{N}_{\mathrm{F} / \mathrm{N}}$ \\
Traits Used &
\end{tabular}

Table 2.3. Results of classification tree analysis. $\mathrm{ROS}$ and $\triangle \Psi \mathrm{M}$ traits reflect average maximum fluorescence values. Misclassification rate and $R^{2}$ are the mean values from five separate runs of the classification tree using identical parameters (see text). (Top) The three phylogenetic clades of $C$. briggsae (Fig. 2.1A) were used as grouping variables during tree construction. (Bottom) Four categories based on isolate-specific nad5 $\Delta$ heteroplasmy level (Fig. 2.1A) were used as grouping variables.

$\underline{\text { Possible relationships between } n a d 5 \Delta \text { and phenotypes }}$

Classification trees were used to determine whether mitochondrial traits distinguished groups of $C$. briggsae isolates with different nad5 $\Delta$ heteroplasmy levels (Fig. 2.1A). Classification trees invariably used ROS and $\triangle \Psi M$ to classify nad5 $\Delta$ 
frequency groups (Table 2.3). In addition to ROS and $\Delta \Psi M$ levels, the phenotypes most often used to classify nad5 $\Delta$ categories were the area of non-functional and functional mitochondrial populations $\left(A_{N P}, A_{F P}\right)$, descriptors of non-functional mitochondrial shape $\left(A_{N}, A R_{N}, C_{N}, C_{N V}\right)$, and the ratio of functional to non-functional mitochondria $\left(N_{F / N}\right)$ (Table 2.3). About $45 \%$ of the total phenotypic variation was accounted for, but misclassification rates were again fairly high ( $33 \%$ on average), meaning that these traits were imperfect predictors of nad5 $\Delta$ category.

As a second means of testing for any association between mitochondrial phenotypes and nad5 $\Delta$ frequency in C. briggsae, individual relationships between all measured traits and isolate-specific nad5 $\Delta$ level were examined. A second order quadratic provided the best fit of many traits to nad5 $\Delta$ percentage (Fig. 2.3; Table 2.4); however, only a minimal amount of the total variation in these traits could be attributed to nad5 $\Delta$ level (low $\mathrm{R}^{2}$ values in Table 2.4). For instance, zero- and high-nad5 $\Delta$ isolates exhibited the highest values for mitochondrial ROS and $\triangle \Psi \mathrm{M}$; however, the relationship between these traits and nad5 $\Delta$ is weak (Fig. 2.3; Table 2.4). All mitochondrial traits describing non-functional organelle shape $\left(A R_{N}, C_{N}, C_{N V}\right)$ exhibited stronger non-linear (quadratic) relationships with nad5 level, as did both measures describing the functional mitochondrial population $\left(A_{F P}, N_{F / N}\right.$ ) (Fig. 2.3; Table 2.4). Neither the mean area of individual non-functional mitochondria $\left(A_{N}\right)$ nor the combined area of these mitochondria $\left(A_{N P}\right)$ was significantly associated with nad5 $\Delta$ percentage in this analysis. However, non-functional mitochondria $\left(A_{N}\right)$ were larger on average than functional 


\begin{tabular}{|c|c|c|c|}
\hline \multirow{2}{*}{ Label } & \multirow{2}{*}{ Trait } & Regression & \multirow{2}{*}{ Equation } \\
\hline & & $r^{2}$ & \\
\hline \multicolumn{4}{|c|}{ Measures of Mitochondrial Physiology } \\
\hline \multirow{2}{*}{$\begin{array}{l}\Delta \Psi \mathrm{M} \\
\text { Mean }\end{array}$} & \multirow{4}{*}{ Membrane potential } & 1.825 & \multirow{2}{*}{$\begin{array}{l}\Delta \Psi \mathrm{M} \text { mean }=907.9-13.12 * \text { nad } 5 \Delta+ \\
0.362 *(\text { nad5 } \Delta-13.96)^{2}\end{array}$} \\
\hline & & 0.02 & \\
\hline \multirow{2}{*}{$\begin{array}{l}\Delta \Psi \mathrm{M} \\
\operatorname{Max}\end{array}$} & & $9.423^{* * *}$ & \multirow{2}{*}{$\begin{array}{l}\Delta \Psi \mathrm{M} \max =2240-38.24 * n a d 5 \Delta+ \\
2.019 *(\text { nad5 } \Delta-13.96)^{2}\end{array}$} \\
\hline & & 0.09 & \\
\hline \multirow{2}{*}{$\begin{array}{l}\text { ROS } \\
\text { Mean }\end{array}$} & \multirow{4}{*}{ Reactive Oxygen Species } & $9.629 * * *$ & \multirow{2}{*}{$\begin{array}{l}\text { ROS mean }=282.8-6.289 * n a d 5 \Delta+ \\
0.330 *(\text { nad } 5 \Delta-14.08)^{2}\end{array}$} \\
\hline & & 0.09 & \\
\hline \multirow{2}{*}{$\begin{array}{l}\text { ROS } \\
\text { Max }\end{array}$} & & $13.43 * * *$ & \multirow{2}{*}{$\begin{array}{l}\text { ROS } \max =1409-34.62 * \text { nad5 } \Delta+ \\
2.293 *(\text { nad5 } 4-14.08)^{2}\end{array}$} \\
\hline & & 0.12 & \\
\hline \multicolumn{4}{|c|}{ Measures of the Mitochondrial Population } \\
\hline \multirow{2}{*}{$\mathbf{A}_{\mathrm{FP}}$} & \multirow{6}{*}{$\begin{array}{l}\text { Area of mitochondrial } \\
\text { population }\end{array}$} & 4.096* & \multirow{2}{*}{$\begin{array}{l}A_{F P}=2766-41.44 * n a d 5 \Delta+2.170 * \\
(\operatorname{nad} 5 \Delta-14.27)^{2}\end{array}$} \\
\hline & & 0.047 & \\
\hline \multirow{2}{*}{$A_{\mathrm{NP}}$} & & 2.299 & \multirow{2}{*}{$A_{N P}=3306-10.77 * n a d 5 \Delta$} \\
\hline & & 0.014 & \\
\hline \multirow{2}{*}{$A_{T P}$} & & 1.689 & \multirow{2}{*}{$\begin{array}{l}\mathrm{A}_{T P}=6083-46.25 * \text { nad5 } \Delta+1.870 * \\
(\text { nad5 } \Delta-14.27)^{2}\end{array}$} \\
\hline & & 0.02 & \\
\hline \multirow{2}{*}{$A_{F P / N P}$} & \multirow{2}{*}{$\begin{array}{l}\text { Ratio of functional to non- } \\
\text { functional mitochondrial } \\
\text { area }\end{array}$} & $5.049 * *$ & \multirow{2}{*}{$\begin{array}{l}\mathrm{A}_{\mathrm{FP} / \mathrm{NP}}=0.974-0.009 * n a d 5 \Delta+ \\
9.431 \mathrm{E}-4 *(\text { nad } 5 \Delta-14.27)^{2}\end{array}$} \\
\hline & & 0.057 & \\
\hline \multirow{2}{*}{$A_{F P / T P}$} & \multirow{2}{*}{$\%$ functional area } & $4.447^{*}$ & \multirow{2}{*}{$\begin{array}{l}\mathrm{A}_{\mathrm{FP} / \mathrm{TP}}=0.421-0.003 * n a d 5 \Delta+ \\
1.928 \mathrm{E}-4^{*}(\text { nad } 5 \Delta-14.27)^{2}\end{array}$} \\
\hline & & 0.051 & \\
\hline \multirow{2}{*}{$\mathrm{N}_{\mathrm{F}}$} & \multirow{6}{*}{ Number of mitochondria } & $5.671 * *$ & \multirow{2}{*}{$\begin{array}{l}\mathrm{N}_{\mathrm{F}}=63.50-0.890 * \text { nad } 5 \Delta+ \\
0.047 *(\text { nad } 5 \Delta-14.27)^{2}\end{array}$} \\
\hline & & 0.064 & \\
\hline \multirow{2}{*}{$N_{N}$} & & 1.416 & \multirow{2}{*}{$\begin{array}{l}\mathrm{N}_{\mathrm{N}}=60.86+0.201 * \operatorname{nad} 5 \Delta- \\
0.015^{*}(\text { nad } 5 \Delta-14.27)^{2}\end{array}$} \\
\hline & & 0.017 & \\
\hline $\mathrm{N}_{T}$ & & 1.28 & $\mathrm{~N}_{\mathrm{T}}=124.4-0.689 *$ nad $5 \Delta+$ \\
\hline $\mathrm{IV}_{\mathrm{T}}$ & & 0.015 & $0.032 *(\text { nad } 5 \Delta-14.27)^{2}$ \\
\hline
\end{tabular}




\begin{tabular}{|c|c|c|c|}
\hline \multirow{2}{*}{$\mathbf{N}_{\mathrm{F} / \mathrm{N}}$} & \multirow{2}{*}{$\begin{array}{l}\text { Ratio of functional to non- } \\
\text { functional mitochondria }\end{array}$} & $5.545^{* *}$ & \multirow{2}{*}{$\begin{array}{l}\mathrm{N}_{\mathrm{F} / \mathrm{N}}=1.183-0.013 * \operatorname{nad} 5 \Delta+ \\
0.001 *(\operatorname{nad} 5 \Delta-14.27)^{2}\end{array}$} \\
\hline & & 0.062 & \\
\hline \multirow{2}{*}{$N_{F / T}$} & \multirow{2}{*}{$\%$ functional mitochondria } & $8.445^{* * *}$ & \multirow{2}{*}{$\begin{array}{l}\mathrm{N}_{\mathrm{F} / \mathrm{T}}=0.492-0.005 * \text { nad } 5 \Delta+2.457 \mathrm{E}- \\
4 *(\text { nad } 5 \Delta-14.27)^{2}\end{array}$} \\
\hline & & 0.092 & \\
\hline \multicolumn{4}{|c|}{ Measures of Individual Mitochondrial Shape } \\
\hline \multirow{2}{*}{$A_{F}$} & \multirow{4}{*}{$\begin{array}{l}\text { Area of individual } \\
\text { mitochondria }\end{array}$} & 0.006 & \multirow{2}{*}{$\begin{array}{l}\mathrm{A}_{\mathrm{F}}=40.70+0.014 * \text { nad } 5 \Delta-5.115 \mathrm{E}- \\
4 *(\operatorname{nad} 5 \Delta-14.27)^{2}\end{array}$} \\
\hline & & $7.20 \mathrm{E}-05$ & \\
\hline \multirow{2}{*}{$A_{N}$} & & 1.931 & \multirow{2}{*}{$\begin{array}{l}A_{N}=56.90-0.342 * n a d 5 \Delta+ \\
0.012 *(\text { nad } 5 \Delta-14.27)^{2}\end{array}$} \\
\hline & & 0.02 & \\
\hline \multirow{2}{*}{$A R_{F}$} & \multirow{4}{*}{ Aspect ratio } & 1.489 & \multirow{2}{*}{$\begin{array}{l}\mathrm{AR}_{\mathrm{F}}=1.689+1.005 \mathrm{E}-3 * \text { nad } 5 \Delta- \\
1.093 \mathrm{E}-4 *(\text { nad } 5 \Delta-14.27)^{2}\end{array}$} \\
\hline & & 0.018 & \\
\hline \multirow{2}{*}{$\mathbf{A R}_{\mathrm{N}}$} & & $7.174 * * *$ & \multirow{2}{*}{$\begin{array}{l}A R_{N}=1.824-0.003 * n a d 5 \Delta+2.864 E- \\
4^{*}(\operatorname{nad} 5 \Delta-14.27)^{2}\end{array}$} \\
\hline & & 0.079 & \\
\hline \multirow{2}{*}{$A R_{F V}$} & \multirow{4}{*}{ Aspect ratio variance } & 1.552 & \multirow{2}{*}{$\mathrm{AR}_{\mathrm{FV}}=0.732-0.006^{*} n a d 5 \Delta$} \\
\hline & & 0.009 & \\
\hline \multirow{2}{*}{$\mathrm{AR}_{\mathrm{NV}}$} & & 1.216 & \multirow{2}{*}{$\begin{array}{l}\mathrm{AR}_{\mathrm{NV}}=1.086-0.009 * n a d 5 \Delta+9.472 \mathrm{E}- \\
4 *(\operatorname{nad} 5 \Delta-14.46)^{2}\end{array}$} \\
\hline & & 0.015 & \\
\hline \multirow{2}{*}{$C_{F}$} & \multirow{4}{*}{ Circularity } & 2.387 & \multirow{2}{*}{$\begin{array}{l}\mathrm{CF}=0.872-5.033 \mathrm{E}-5^{*} \text { nad } 5 \Delta- \\
2.916 \mathrm{E}-5^{*}(\text { nad } 5 \Delta-14.27)^{2}\end{array}$} \\
\hline & & 0.028 & \\
\hline \multirow{2}{*}{$\mathrm{C}_{\mathrm{N}}$} & & 4.314* & \multirow{2}{*}{$\begin{array}{l}\mathrm{C}_{\mathrm{N}}=0.827+9.483 \mathrm{E}-4 * n a d 5 \Delta- \\
4.894 \mathrm{E}-5 *(\text { nad } 5 \Delta-14.27)^{2}\end{array}$} \\
\hline & & 0.049 & \\
\hline \multirow{2}{*}{$\mathrm{C}_{\mathrm{FV}}$} & \multirow{4}{*}{ Circularity variance } & 0.888 & \multirow{2}{*}{$\begin{array}{l}\mathrm{C}_{\mathrm{FV}}=0.038+1.829 \mathrm{E}-4 * \text { nad } 5 \Delta- \\
1.096 \mathrm{E}-5 *(\text { nad } 5 \Delta-14.01)^{2}\end{array}$} \\
\hline & & 0.011 & \\
\hline \multirow{2}{*}{$\mathrm{C}_{\mathrm{NV}}$} & & $5.208 * *$ & \multirow{2}{*}{$\begin{array}{l}\mathrm{C}_{\mathrm{NV}}=0.050-3.02 \mathrm{E}-5^{*} n a d 5 \Delta+ \\
2.874 \mathrm{E}-5 *(\operatorname{nad} 5 \Delta-14.46)^{2}\end{array}$} \\
\hline & & 0.06 & \\
\hline
\end{tabular}

Table 2.4. Assigned labels of all mitochondrial traits measured for $\boldsymbol{C}$. briggsae natural isolates. The test statistic and adjusted $r^{2}$ values for the best fit regression of each phenotype to nad $5 \Delta$ frequency are given for each trait. Bold font identifies the nine traits retained in the classification tree analysis when using categories based on isolate-specific nad5 5 \% (see Table 2.3). Italicized values indicate that a linear regression provided the best fit to $n a d 5 \Delta \%$, while standard text denotes a quadratic 
relationship to $\operatorname{nad} 5 \Delta \%{ }^{*}, * *$, and ${ }^{* * *}$ denote $\mathrm{p}<0.05,0.01,0.001$, respectively. Subscripts $\mathrm{N}, \mathrm{F}$, and $T$ indicate whether the measure refers to Non-functional, Functional, or Total mitochondria. Subscript $P$ and $V$ denote that the measure refers to the entire mitochondrial population (not individual mitochondria), or the average individual variance in that trait, respectively.

mitochondria $\left(A_{F}\right)$ (Table 2.1). Overall, nad5 load was more strongly associated with descriptors of the shape of non-functional mitochondria and traits describing the total population of functional mitochondria than with those describing any aspect of functional mitochondrial shape.

\section{Insights from mitochondrial-nuclear hybrid lines}

To directly test whether the observed differences among C. briggsae isolates were due to mtDNA variation, we examined the nine phenotypes retained in classification tree analyses for two mitochondrial-nuclear hybrid strains (e.g., Fig. 2.4). If among-isolate variation in mitochondrial phenotypes is primarily due to an additive mtDNA genetic contribution, a correspondence between mitochondrial-nuclear hybrid strain phenotypes and their respective mitochondrial parental strain is predicted.

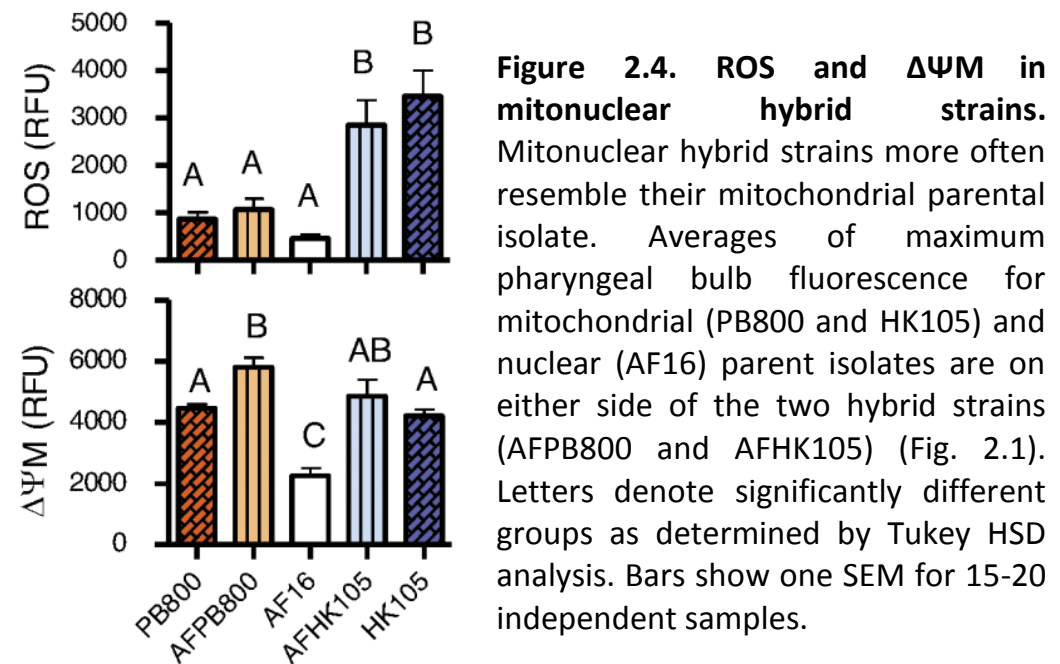

Strain 
Consistent with this idea, hybrid strains were more similar to their mitochondrial parent strains than to their nuclear parent strains (Table 2.5). Considering ROS and $\triangle \Psi M$ only, the hybrid strains were more similar to their mitochondrial parent in each case (Fig. 2.4). Note that in the AF16 and PB800 pairing, the hybrid is not significantly different from the nuclear parent, but is still more similar to the mitochondrial parent. The diversity in ROS and $\triangle \Psi M$ phenotypes seen in the three strains studied therefore appears to be due to variation in mtDNA as opposed to among-strain nuclear variation. However, the relative contribution of each genome was less clear for measures of the mitochondrial population and mitochondrial shape. These traits rarely differed significantly among parent and hybrid strains studied here (Table 2.5), although a larger study may have revealed small differences. A visual inspection of these data suggested that mitochondrial morphology traits may be more affected by the nuclear genome (hybrids are more similar to the paternal isolate) or by mitochondrial-nuclear epistasis (hybrids differ from both parental isolates), than were ROS and $\triangle \Psi M$ (not shown).

\begin{tabular}{|c|c|c|c|c|}
\hline $\begin{array}{c}\text { Hybrid and } \\
\text { Mitochondrial } \\
\text { Parent }\end{array}$ & Difference & Trait & Difference & $\begin{array}{l}\text { Hybrid and } \\
\text { Nuclear } \\
\text { Parent }\end{array}$ \\
\hline \multirow{5}{*}{ AF-PB and PB } & 207.7 & ROS Max & 612.1 & \multirow{5}{*}{$A F-P B$ and $A F$} \\
\hline & $1333^{*}$ & $\Delta \Psi M$ Max & $3543 * * *$ & \\
\hline & 1.34 & $\begin{array}{l}\text { Area of non-functional } \\
\text { mitochondria }\end{array}$ & 5.791 & \\
\hline & -1113 & $\begin{array}{l}\text { Area of the non- } \\
\text { functional mitochondrial } \\
\text { population }\end{array}$ & -450.2 & \\
\hline & -1894 & $\begin{array}{l}\text { Area of the functional } \\
\text { mitochondrial population }\end{array}$ & 653.5 & \\
\hline
\end{tabular}




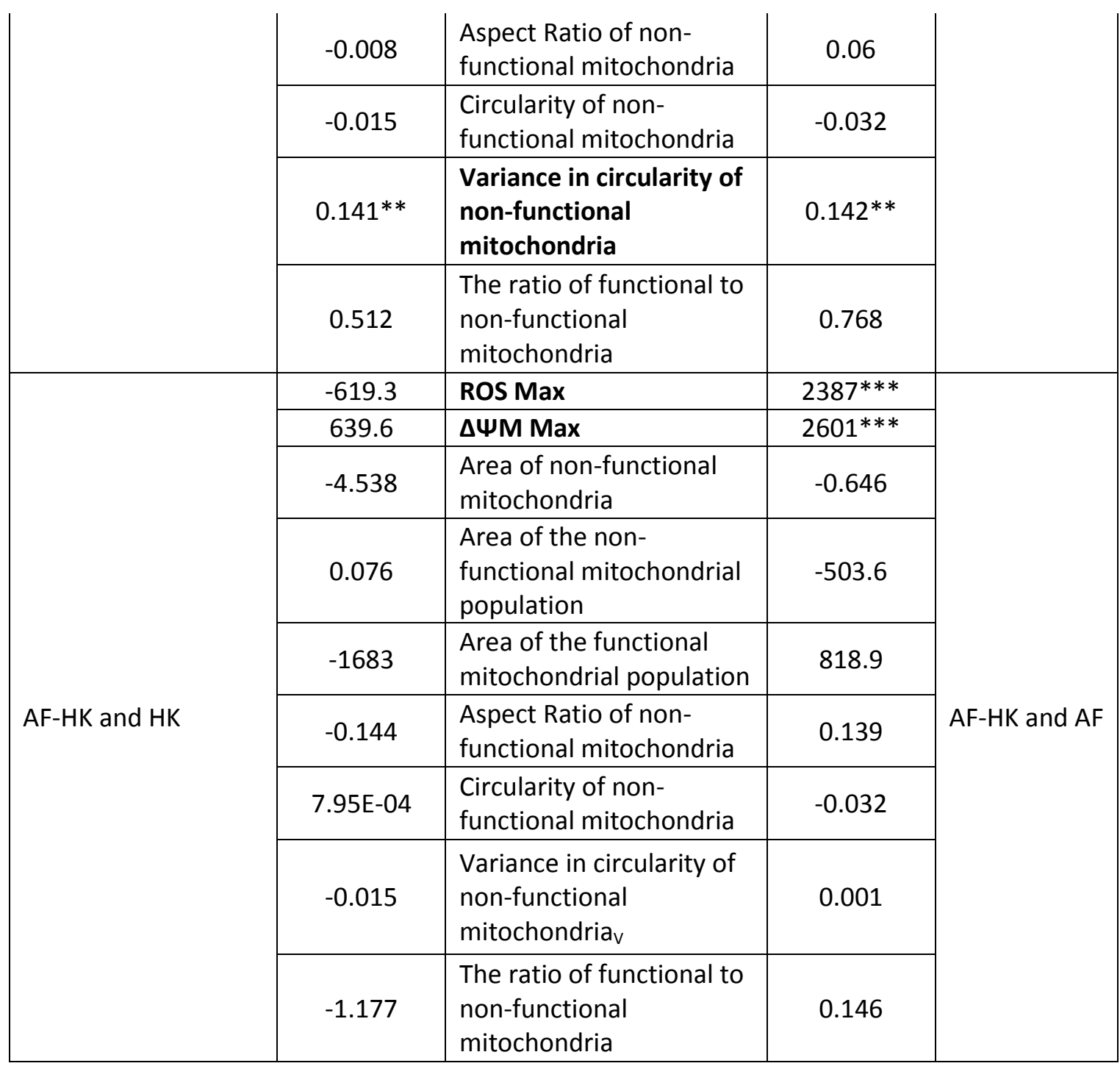

Table 2.5. Comparison of mitonuclear hybrid strains to parent strains. The difference between the means of each pair of mitonuclear hybrids and their parental isolates are shown. Bolded traits exhibit significant differences among hybrid and parent strains. ${ }^{*}, * *$, and $* * *$ denote $\mathrm{p}<0.05,0.01$, and 0.001 respectively (Tukey HSD, $\alpha=0.05$ ). AF $=$ AF16, PB $=$ PB800, HK $=$ HK105.

\section{Discussion}

\section{Evolutionary implications}

We have used in vivo techniques with $C$. briggsae nematodes to conduct the first analysis of naturally-occurring variation in mitochondrial function, morphology, and 
properties of mitochondrial populations. We discovered considerable variation among natural C. briggsae isolates for most of the measured traits. In fact, we find more variation among natural isolates of $C$. briggsae for both ROS and $\triangle \Psi \mathrm{M}$ than has been measured among ETC mutant strains of $C$. elegans (Dingley et al. 2009; Lemire et al. 2009). This result is partly explained by the greater phenotypic variation observed within C. elegans mutant strains (e.g., see among-replicate variation in Figs. 2 and 3 in Dingley et al., 2009) than within C. briggsae isolates; the source of this species difference is unclear. Our findings also mirror those of previous studies revealing extensive variation in life-history and metabolic traits among the same isolates (Howe and Denver 2008; Estes et al. 2011). A good deal of this variation was related to the phylogeographic clade membership of particular isolates (Fig. 2.3; Table 2.2); this was especially true for $\Delta \Psi \mathrm{M}$ (Fig. 2.3G; Table 2.3). C. briggsae is globally distributed (Sudhaus and Kiontke 2007) and both mitochondrial and nuclear genetic analyses consistently reveal the existence of distinct phylogeographic clades that are separated by latitude (Howe and Denver 2008; Cutter et al. 2010). These clades have experienced different population genetic histories (Cutter et al. 2006, 2010; Howe and Denver 2008) and show some evidence for local adaptation to temperature; e.g., Tropical clade isolates appear to have higher thermal maxima (Prasad et al. 2011). Consequently, divergence in mitochondrial phenotypes measured here may reflect clade-specific phylogenetic or selective histories. It is impossible to say without further study whether the among-clade differences in mitochondrial function measured here have an adaptive significance or are a non- or 
maladaptive consequence of genetic drift (Howe and Denver 2008); however, it is conceivable that Tropical $C$. briggsae isolates adaptively maintain low $\triangle \Psi \mathrm{M}$. Because $C$. briggsae is ectothermic, its biology will be driven strongly by environmental temperature. At higher temperatures, biological reactions such as those controlling electron transport and the subsequent production of ROS occur at higher rates (Hochachka and Somero 2002). The higher temperatures experienced by Tropical clade isolates may have thus subjected them to higher ROS levels during their evolutionary history. Reducing ROS production, especially from complex I, may be achieved by lowering $\triangle \Psi M$ by uncoupling oxidative phosphorylation (Brand 2000; Murphy 2009). We may therefore hypothesize that Tropical $C$. briggsae have adaptively reduced their $\triangle \Psi \mathrm{M}$ to counter increased ROS levels brought on by higher temperatures and perhaps exacerbated by nad $5 \Delta$-induced complex I inefficiency.

Although other traits appear to be influenced by both nuclear and mitochondrial genetic contributions, our data are consistent with the interpretation that much of the among-isolate variation in mitochondrial ROS and $\Delta \Psi \mathrm{M}$ can be attributed to mitochondrial genome content rather than to nuclear divergence among isolates (Fig. 2.4; Table 2.5). In agreement with this finding, ROS level and $\Delta \Psi \mathrm{M}$ were shown to be the best predictors among those tested of nad5 $\Delta$ category in classification analyses (Table 2.3). Because mitochondrial electron transport relies upon the coordinated functioning of both mitochondrial and nuclear encoded ETC components, there is ample opportunity for epistatic interactions between mtDNA and nuclear genomes (Ellison and 
Burton 2006, 2010; Ellison et al. 2008; Arnqvist et al. 2010). A recent analysis of recombinant inbred lines generated from reciprocal crosses between $C$. briggsae isolates AF16 and HK104 provides indirect evidence for such mitochondrial-nuclear incompatibilities (Ross et al. 2011). In agreement with this result, we observed extensive paternal transmission of mitochondria in other sets of $C$. briggsae mitonuclear hybrid strains (Materials and Methods). It could therefore have easily been the case that $C$. briggsae hybrids studied here bore no similarity to either parental isolate as a result of interpopulation hybrid breakdown. This is particularly true for our hybrid strains, both of which resulted from crosses between a Tropical and a Temperate isolate (Fig. 2.1). That the results of our hybrid strain analyses for ROS and $\triangle \Psi M$ are consistent with an additive effect of the mitochondrial genome suggests that the natural isolates used to generate the hybrids have either not experienced functional divergence for the relevant ETC gene products or have purged variants that generate deleterious epistatic interactions before they had the opportunity to create fixed polymorphisms between isolates (c.f. Montooth et al., 2010). An analysis of nucleotide diversity at nonsynonymous versus synonymous codon positions $\left(\pi_{\mathrm{a}} / \pi_{\mathrm{s}}\right)$ in ten mtDNA-encoded ETC genes among 22 C. briggsae natural isolates suggested that these genes are under purifying selection ( $\pi_{\mathrm{a}} / \pi_{\mathrm{s}}<1$ for all genes) and have not likely experienced functional divergence (Howe and Denver 2008). Patterns of diversity and divergence at nuclearencoded ETC genes have not been analyzed in C. briggsae natural isolates. In either 
case, our results suggest that these particular hybrid strains will be valuable for future studies of mitochondrial function.

While results of the hybrid analyses showed that ROS and $\triangle \Psi \mathrm{M}$ were influenced by mtDNA content, analyses of the natural $C$. briggsae isolates suggested that at least a portion of the among-isolate phenotypic variation may be associated with nad5 $\Delta$ heteroplasmy level or other factors in linkage disequilibrium with nad5 $\Delta$ (Fig. 2.3; Table 2.4). In particular, several traits exhibited non-linear relationships with isolate-specific nad5 $\Delta$ level, a pattern in agreement with previous findings for these isolates (Estes et al. 2011); however, the patterns are in most cases quite weak (Table 2.4; Fig. 2.3). Our results for highest-deletion isolate HK105 are of special note, however, since this isolate exhibits the highest ROS levels (Figs. 2.3 and 2.4) and the lowest reproductive fitness (See Figure 2A in Estes et al. 2011), it may be that HK105 (>50\% deletion bearing genomes) has reached a threshold beyond which nad5 $\Delta$ elicits deleterious effects; i.e., high ROS levels associated with extreme ETC dysfunction (Rossignol et al. 2003). Congruent with this idea, HK105 also had the largest ratio of functional to nonfunctional organelles (Fig. 2.3H) and the smallest area of depolarized mitochondria (Fig. 2.3B) compared to other isolates. One interpretation of these data is that mitochondria with nad5 $\Delta$ loads beyond $\sim 50 \%$ are unable to rescue functionality through mitochondrial fusion (Chen et al. 2007; Schon et al. 2010) and the ensuing mitophagic degradation of highly impaired organelles increases the ratio of functional to non- 
functional organelles. A direct test of this hypothesis awaits development of techniques for simultaneously genotyping and phenotyping individual mitochondria.

It has been proposed that lowering mitochondrial $\Delta \Psi M$ may slow the rate of ROS production and help to alleviate oxidative stress - and perhaps extend lifespan (Brand 2000; Lemire et al. 2009; Murphy 2009). Based on such studies and on the fact that ROS and $\triangle \Psi \mathrm{M}$ were most important for classifying our $C$. briggsae natural isolates into nad5 $\Delta$ categories (Table 2.3), we expected that a statistical relationship between these traits might emerge, but observed no such correlation (Figs. 2.3G, 2.3I). A caveat that prohibits further interpretation of this result is that ROS and $\triangle \Psi M$ levels were necessarily measured on different individual nematodes; estimates of each trait were therefore obtained from different sets of mitochondria (Materials and Methods). (Conversely, $\triangle \Psi M$ and morphology assays were conducted on the same individuals.) Unfortunately, to our knowledge, all ROS and $\Delta \Psi M$ probes utilize nearly identical fluorescent spectra, making simultaneous in vivo quantification of both traits impossible. It is noteworthy, however, that the results of our studies differ from those of Lemire et al. (2009), which showed that reduced $\Delta \Psi M$ was associated with increased lifespan across four classes of $C$. elegans longevity mutants. We find no obvious relationship between isolate-specific $\Delta \Psi \mathrm{M}$ and lifespan measured in a previous study [47]. In particular, the long-lived PB800 isolate (Estes et al. 2011) was observed here to have a high $\triangle \Psi \mathrm{M}$ (Fig. 2.2G). Further study would be required to understand why this isolate deviates from the strong pattern seen in C. elegans experimental strains. A 
possible explanation may lie in the nuclear genetic differences among the $C$. briggsae isolates used here; in contrast, Lemire (2009) studied C. elegans mutants on an otherwise common nuclear background.

The analyses of mitochondrial phenotypes presented here also identified traits that diagnose mitochondrial functionality. Apart from ROS, $\triangle \Psi M$, and $A_{F P}$ (combined area of the functional mitochondrial population), traits retained in classification trees all described some aspect of depolarized mitochondria (Table 2.3). In other words, descriptors of depolarized mitochondria provided more information about the nad5 $\Delta$ frequency class to which natural isolates belonged than did descriptors of functional mitochondria. For example, although isolates did not differ in any aspect of functional mitochondrial shape (Table 2.1), traits describing non-functional mitochondrial shape $\left(A R_{N}, C_{N}\right.$, and $\left.C_{N V}\right)$ were retained in classification trees; these traits also showed

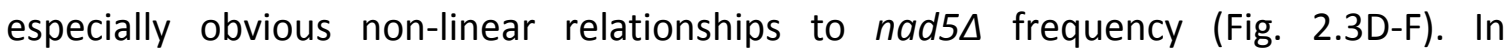
particular, depolarized mitochondria tended to be larger (Fig. 2.3D) and more variable with respect to circularity (Fig. 2.3F) (and consequently less circular, Fig. 2.3E) in zeroand high-nad5 $\Delta$ strains. Furthermore, although some traits describing the average number and combined area of the functional mitochondrial population were found to be (mostly non-linearly) related to nad5 frequency in our regression analyses (Table 2.4), traits describing individual functional mitochondria were never found to be associated with nad5 $\Delta$ level in any analysis. Taken together, our results suggest that isolates with different nad5 levels are more variable with respect to their depolarized 
mitochondrial populations than to their polarized mitochondrial populations. Furthermore, we found that functionality (based on $\triangle \Psi \mathrm{M}$ ) is strongly associated with elongated organelles, whereas non-functionality is associated with circular (i.e., fragmented) organelles. This result is in agreement with previous findings (Chan 2006a,b; Duvezin-Caubet et al. 2006; Schon et al. 2010) and with the idea that damaged mitochondria lose $\triangle \Psi M$ and undergo fragmentation early in the cellular apoptosis process (Okamoto and Shaw 2005).

\section{Conclusions and outlook}

We have reported a novel analysis of subcellular processes in C. briggsae that, to our knowledge, provides the first explicit treatment of within-species natural variation in form and function of an organelle. Through the use of mitochondrial-nuclear hybrid lines, we demonstrated that mtDNA genotype is a strong driver of a portion of this natural variation. We also found evidence for complex associations between mitochondrial nad5 $\Delta$ frequency and mitochondrial functioning (ROS and $\triangle \Psi M$ ) and morphology traits. Although our study represents a major step forward in understanding natural variation in subcellular processes in vivo, additional work is required to move beyond correlative associations between mitochondrial genotypes and phenotypes to a direct determination of the genetic underpinnings of subcellular variation. Achieving this goal in our system would necessitate the simultaneous genotyping (e.g., through mitochondrial mRNA or DNA labeling) and phenotyping of individual mitochondria, which is not possible currently. 
More generally, our study indicates that evolutionary approaches hold promise for advancing our knowledge of mitochondrial population dynamics and other cell-level processes. Although there is a long history of evolutionary analysis providing key insights into DNA-level genetic processes and organismal phenotypes, phenomena at more intermediate levels of biological organization (e.g., cellular, subcellular) have largely been overlooked by evolutionary biologists during the last century. Likewise, cell biology research rarely considers natural within-species variation. Evolutionary cell biology is an emerging and essentially untapped research area in need of both theoretical and empirical work. With regard to mitochondria, outstanding questions in this discipline include: what is the role of mitochondrial fission and fusion in purging deleterious heteroplasmic mtDNA mutations, how do epistatic interactions between nuclear and mtDNA subunits of ETC genes affect mitochondrial form and function and organismal fitness, and how do we analyze the mode and strength of selection on mitochondrial form and function? Together with the many other advantages to the $C$. briggsae system, its amenability to in vivo physiological studies and abundant amongisolate mtDNA variation suggest that this species will be a valuable natural model system for addressing such questions. 


\section{CHAPTER THREE}

Evolution of mitochondrial heteroplasmy and ROS levels under genetic drift in experimental lines of Caenorhabditis briggsae

\section{Introduction}

Mutation is a fundamental evolutionary process that underlies genetic change in all populations. It is the ultimate source of all molecular and quantitative genetic variation and plays a fundamental role in the susceptibility and generation of human disease. Accurate measures of the rates, molecular mechanisms, and distributions of fitness effects of mutations are critical for many applications of evolutionary theory including: inferring evolutionary relationships, testing for selection on molecular sequence, estimating effective population size from standing levels of neutral genetic variation, parameterizing many population genetic models (Lynch et al. 1999) and predicting long-term patterns of phenotypic evolution (Jones et al. 2007). Accordingly, much effort has been devoted to studying the rates and molecular underpinnings of spontaneous mutation in a variety of organisms (Lynch et al. 1999; Halligan and Keightley 2009; Kondrashov and Kondrashov 2010). Much of this work has been achieved using laboratory mutation-accumulation (MA) experiments - first envisioned by Hermann Muller (Muller 1928) and employed by Terumi Mukai (Mukai 1964) - that seek to distinguish the impact of mutation from that of other evolutionary forces affecting rates of molecular evolution. These experiments allow mutations to accrue free from selection in the genomes of replicate lineages (MA lines) maintained by 
extreme inbreeding across many generations (Halligan and Keightley 2009). Because different numbers and types of mutations will accumulate in such lines, and because most new mutations with phenotypic consequences are deleterious for organismal fitness, it is expected that mean fitness and related phenotypes will decline linearly with successive generations of MA and that the phenotypic variance among MA lines will increase simultaneously (e.g., Fig. 5 in Mukai 1964). The changes in mean phenotypes and among-line variance in MA lines compared to their progenitors can be used to make indirect estimates of deleterious rates and mutational effects; i.e., the Bateman-Mukai approach (Lynch et al. 1999; Halligan and Keightley 2009).

A few MA experiments have now been combined with DNA sequencing to provide direct estimates of the average per-generation rate of single-nucleotide substitutions arising in nuclear (nDNA) and mitochondrial (mtDNA) genomes(Denver et al. 2000, 2009, 2012; Lynch et al. 2008; Keightley et al. 2009; Ossowski et al. 2010). Direct estimates from mitochondrial (mtDNA) genomes have yielded mutation rates $\sim 10 \mathrm{x}$ higher than nuclear rates and show evidence for variation in the underlying mutational processes among and within species (Montooth and Rand 2008). Importantly, few studies (Haag-Liautard et al. 2008; Howe et al. 2010; Clark et al. 2012) have attempted to evaluate mtDNA heteroplasmy (multiple mtDNA haplotypes coexisting within an individual), but have described fixed (i.e., homoplasmic) or nearlyhomoplasmic mutations that have already navigated the poorly understood intracellular adaptive landscape to achieve high frequency. Recent work demonstrates that mutant 
mtDNA molecules have the capacity to act as selfish elements (Clark et al. 2012), perhaps driven by a replicative advantage for deletion mutations due to their small size. Such a phenomenon will directly affect the evolution of mtDNA heteroplasmy levels. Clark, et al. (2012) found a consistent increase in the heteroplasmy level of deletionbearing genomes within lines of Caenorhabditis briggsae subjected to ten generations of MA treatment. Furthermore, many features of mitochondrial biology are likely to have consequences for mtDNA mutation dynamics distinct from those affecting nDNA mutations. These include: maternal inheritance of mtDNA (small effective population size), the organelle bottleneck that occurs during fertilization (Stewart et al. 2008), and the complex mitochondrial life cycle involving organelle fission, fusion and autophagy that may act to purge damaged mtDNA genomes (Twig et al. 2008b; Kuznetsov and Margreiter 2009; Kowald and Kirkwood 2011; Bess et al. 2012; Meyer and Bess 2012). The complexity of mitochondrial population dynamics and our incomplete understanding of the relative influence of evolutionary forces acting on mtDNA genomes and traits imply that MA studies have the potential to provide significant insight into these processes.

Although MA studies have provided some information on the rates and molecular consequences of mtDNA mutation, the consequences of MA on cell- or organelle-level phenotypes have not been addressed. Knowing how such phenotypes evolve in the absence of natural selection can improve our understanding of the role of natural selection in maintaining wildtype phenotypes in natural populations. Proper 
mitochondrial functioning is essential for organismal health and survival since the majority of the energy needed for most eukaryotic life is produced by the mitochondrial electron transport chain (ETC). However, the ETC also creates ROS as a byproduct of oxidative phosphorylation (Raha and Robinson 2000). If permitted to accumulate, ROS can damage important cellular macromolecules including proteins and nucleic acids (Wanagat et al. 2001; Yang et al. 2007), a situation often induced by the impairment of the ETC (Grad and Lemire 2004; Verkaart et al. 2007; Dingley et al. 2009). One could therefore hypothesize that ROS levels will increase monotonically with generations of MA. This prediction is supported by the "vicious cycle theory," which is an extension of mitochondrial free radical theory of aging (Harman 1956). The free radical theory of aging states that the reactive byproducts (including ROS) of mitochondrial oxidative phosphorylation will damage biological macromolecules over the lifetime of organism, resulting in age-related deterioration (Harman 1956). The vicious cycle theory further hypothesizes that the oxidative damage to DNA, lipids, and proteins will in turn reduce the stability or efficiency of mitochondria leading to exponentially more ROS production (Bandy and Davison 1990). A plausible alternative is that ROS levels would exhibit an eventual decline if the mitochondrial ETC becomes too damaged to function. These ideas are intensely debated in the aging biology literature (Jacobs 2003; Pak et al. 2003; Trifunovic et al. 2005; Gruber et al. 2008; Selman et al. 2012) and have stimulated considerable research on the relationships between ROS and somatic mutation (Hartman et al. 2001; Wei and Lee 2002; Trifunovic et al. 2005; Hiona and 
Leeuwenburgh 2008; Khrapko and Vijg 2009). However, few cross-generational studies have been conducted; we therefore have almost no information on the relationships between ROS and heritable germline mutation rates or dynamics.

Caenorhabditid nematodes have become popular models for studying a variety of questions in evolutionary biology including the genome-wide rates and patterns of spontaneous mutation and for separate investigations of the effects of mitochondrial ETC mutations and organelle dysfunction. Here, we take advantage of the well-studied C. briggsae nematode system to integrate these approaches and examine how mitochondrial ROS and nad5 5 heteroplasmy levels evolve during MA. A previous MA study of three rhabditid species revealed a faster rate of fitness degradation in $C$. briggsae compared to other nematode species (Baer et al. 2005). The C. briggsae MA lines were later observed to acquire mitochondrial genome deletions at a much higher rate than in C. elegans (Howe et al. 2010). Additionally, a large mtDNA deletion, nad54, was discovered to persist in natural populations of $C$. briggsae, including the two isolates studied by Baer, et al. (2005) (Howe and Denver 2008). Levels of nad5 heteroplasmy - the average fraction of nad5 $\Delta$-bearing genomes within an individual ranges from 0 to over $50 \%$ among natural isolates of $C$. briggsae (Howe and Denver 2008; Estes et al. 2011). Although nad5 $\Delta$ appears to have negative consequences for nematode health and fitness at high (> 40\%) heteroplasmy levels (Howe and Denver 2008; Estes et al. 2011), its presence appears unrelated to isolate-specific variation in net ROS level or other aspects of mitochondrial functioning (Hicks et al. 2012). 
We conducted a mutation-accumulation experiment using experimental lines generated from geographically distinct isolates and one mitochondrial-nuclear hybrid strain of Caenorhabditis briggsae to examine the effects of extreme genetic drift on the levels of mitochondrial ROS and nad5 $\Delta$ heteroplasmy levels. We observed substantial evolution of both characters across $\leq 50$ generations of MA. Mean mitochondrial ROS evolved in a remarkably linear fashion among MA lines generated from the $C$. briggsae natural isolates, but non-linearly among lines of the mitonuclear hybrid strain. However, the patterns of ROS evolution, as well as the patterns of among-line variance in ROS level, were isolate-specific and did not support the hypothesis that ROS levels will necessarily increase with mutational load. Additionally, we observed a tendency for nad5 $\Delta$ levels to increase with MA in agreement with the idea that nad5 $\Delta$-bearing mitochondrial genomes are transmitted as selfish elements (Clark et al. 2012), but we also find compelling evidence for mitochondrial threshold effects acting on nad5 $\Delta$ heteroplasmy levels. Finally, we found no relationship between the patterns of evolution in ROS and nad5 levels. We discuss our results in light of the unique biology of mitochondria and the implications of this study for mitochondrial mutation biology.

\section{Materials and Methods}

\section{Nematode strains}

For all assays, we used three well-studied natural C. briggsae isolates (Howe and Denver 2008; Estes et al. 2011; Clark et al. 2012; Hicks et al. 2012) and one experimental 
mitochondrial-nuclear hybrid strain. The three natural isolates (ED3101, AF16, and HK105) were chosen to represent three of the major C. briggsae phylogeographic clades (Cutter et al. 2006, 2010), to encompass much of the known phenotypic variation in lifehistory and metabolic traits (Estes et al. 2011; Hicks et al. 2012) and to span the known range of nad5 genotypic diversity for this species (Howe and Denver 2008; Clark et al. 2012) (Figs. 2.1, 3.1). The formation of nad5 $\Delta$ requires an upstream pseudogene called

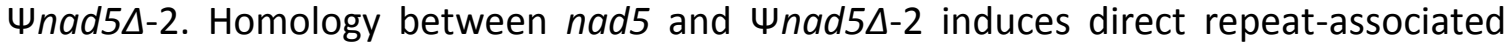
deletion events that generate nad5 $\Delta$-bearing genome (See Figure 1 in Howe and Denver, 2008). Thus, isolates without the $\Psi$ nad5 $\Delta-2$ pseudogene (e.g., ED3101) harbor only intact mitochondrial genomes, while isolates containing $\Psi$ nad5 $\Delta-2$ have nad5 $\Delta$ heteroplasmy levels ranging from zero to over 50\% (Fig. 3.1). For further details regarding the $C$. briggsae natural isolates and the transmission genetics of nad5 $\Delta$ please see (Howe and Denver 2008; Estes et al. 2011).

The experimentally generated hybrid strain ("ED-AF") contains the mitochondrial

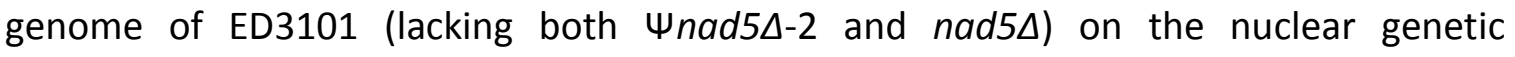
background of AF16 (whose mitochondrial genome contains both elements). These hybrid strains were generated through serial backcrossing of AF16 males to hermaphrodite cross-progeny for 10 generations. This process is expected to result in worms with $>99.9 \%$ of their nuclear genome from AF16 and their mitochondrial genome from ED3101. The nuclear contribution was confirmed using isolate-specific amplicon size differences of the third intron of the Cbr-polh-1 gene and agarose gel 
A

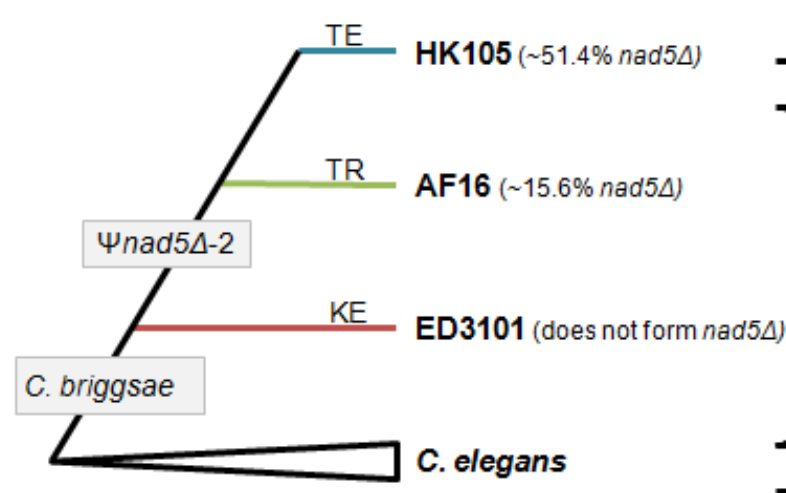

B

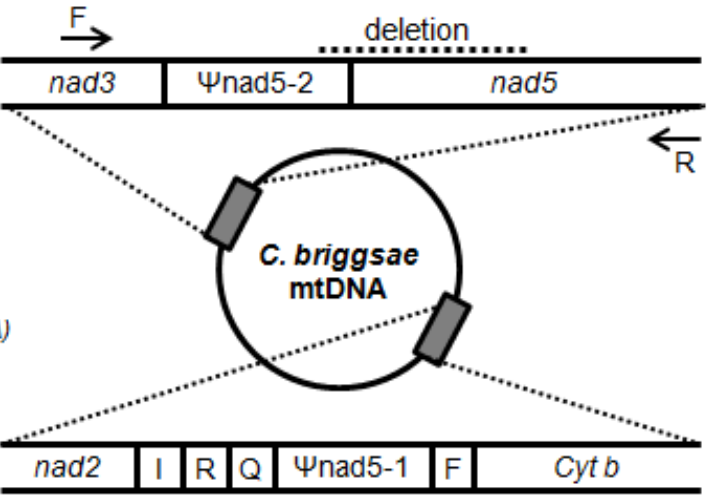

Figure 3.1. C. briggsae natural isolates and description of the nad5 mtDNA deletion. A. Evolutionary relationships and average line-specific nad5 heteroplasmy level for the three $C$. briggsae natural isolates studied here. $\mathrm{KE}=$ Kenya clade; TE and TR $=$ temperate and tropical clades. Gray boxes indicate origin of $C$. briggsae and the $\Psi$ nad5 $5-2$ pseudogene required for nad5 $\Delta$ formation. The mitochondrialnuclear hybrid (ED-AF) contains the mitochondrial contribution from ED3101 on the AF16 nuclear background, and thus should not experience the deletion. B. Positions of the nad5 $\Delta$ deletion (dashed line at top) and $\Psi$ nad5 5 -2 elements within the C. briggsae mitochondrial genome. Arrows indicate primers (adapted from Howe and Denver, 2008).

electrophoresis (Raboin et al. 2010). A combination of PCR with gel analysis and direct sequencing of a portion of the nad5 (Howe and Denver 2008) and COIl (Denver et al. 2003a) genes confirmed that hybrid worms contained the expected mtDNA (Howe and Denver 2008; Raboin et al. 2010; Hicks et al. 2012). Further, hybrid animals were found to share similar nad5 $\Delta$ levels with the original mitochondrial parent through PCR and agarose gel methods described in (Howe and Denver 2008).

\section{Propagation and maintenance of MA lines}

MA lines were generated from the $C$. briggsae progenitor strains described above and indicated in Figure 3.1. The MA lines generated from the three natural $C$. briggsae isolates were a subset of those studied by Clark, et al (2012) who provides a detailed description of the conditions and procedures for the mutation-accumulation 
experiment. MA lines of the ED-AF hybrid strain were propagated concurrently with those from the natural strains (not reported in Clark, et al 2012). Briefly, for each of four progenitor strains, a single hermaphrodite nematode was allowed to self-reproduce, after which $24 F_{1}$ hermaphrodite progeny were individually plated to generate the MA generation zero $\left(\mathrm{G}_{0}\right)$ for a total of $96 \mathrm{MA}$ lines (24 per strain). As described in Clark, et al (2012), the natural strain MA progenitors were not inbred for multiple generations prior to establishment of MA lines; this was done in order to capture any diversity that may have existed in individual nad5 $\Delta$ mtDNA levels and to avoid inflating deletion levels by bottlenecking. Each line was propagated for 50 generations or until extinction via singlenematode bottlenecking as previously described (Clark et al. 2012). All lines were maintained at $20^{\circ} \mathrm{C}$ with Escherichia coli OP50 as a food source. Following each transfer, nematode populations from the previous generation were retained as backups to reinitiate any MA lines that failed to reproduce. Lines were declared extinct if they failed to reproduce after three such attempts. F2 progeny of each MA line were cryogenically preserved using standard protocols (Stiernagle 2006) at ten-generation intervals and/or at the final MA generation prior to line extinction $\left(G_{F}\right)$. Assays for nad5 5 and ROS levels (below) were performed on nematode populations recovered from these frozen stocks. For the current study, a total of $20 \mathrm{MA}$ lines (5 from each C. briggsae strain) were selected at random for these analyses.

\section{$\underline{\text { PCR analysis of nad5 } 5 \text { level }}$}


We assessed the relative levels of intact and nad5 5 -bearing mtDNA genomes in C. briggsae MA lines using a previously described PCR-based assay (Howe \& Denver, 2008). PCR reaction products were electrophoresed on standard $1.5 \%$ agarose gels and digitally recorded as *.TIF files. Gel images were scored based on the three deletionproduct categories described in Clark, et al (2012). PCR products from individuals lacking nad5 $\Delta$ will yield a single, large amplicon. Lines generated from the Temperate and Tropical clade isolates (Fig. 2.1) can potentially generate two discrete amplicons: a larger, intact mtDNA product, and a smaller, deletion-bearing amplicon. Thus, this PCR assay can yield three ordinal results describing relative nad5 $\Delta$ level: 1 ) intact $=$ large band only, 2) intermediate $=$ both small and large bands, 3 ) deletion = small band only. Howe and Denver (2008) found that these three ordinal PCR results correspond to an average nad5 5 deletion frequency of $4 \%, 30 \%$ and $60 \%$ respectively, when assayed using qPCR. The ordinal band-type results reported here were generated from assays conducted on mixed-age populations of thousands of individuals, with one replicate per line for $M A$ generations $G_{0}, G_{10}$ and $G_{F}$. Two lines (ED3101 line 2 and HK105 line 4) were unable to be assayed at $\mathrm{G}_{\mathrm{F}}$ and were therefore excluded from all analyses involving the $\mathrm{G}_{\mathrm{F}}$ nad5 $\Delta$ level.

$\underline{\text { In vivo ROS assays }}$

We used established confocal microscopy techniques for the in vivo quantification of net mitochondrial ROS levels in C. briggsae experimental lines (Dingley et al. 2009; Estes et al. 2011; Hicks et al. 2012) . These assays began with a standard 
bleaching protocol to recover age synchronous worms from cryogenic stocks of each MA line. Worms were incubated for 24 hours prior to imaging in 10uM MitoSOX Red (Molecular Probes Inc., Eugene, OR), a mitochondria-targeted fluorescent dye that measures total levels of mitochondrial oxidants in vivo (Zielonka and Kalyanaraman 2010). Concurrently, a second set of worms from each MA line was incubated under identical conditions without MitoSOX probe to serve as an internal control. In addition, we acquired 4-6 images of a single line, AF16 ( $G_{0} M A$ line 2), during each round of image acquisition. This set of images allowed us to compare relative fluorescence values for one line throughout the entire experiment and to account for any fluctuation in the performance of the imaging system or fluorescent probe.

For each strain, fluorescent z-stack images of the pharyngeal bulb of 40 youngadult stage treatment worms and 9 control worms were acquired at 60X magnification using a high resolution wide field Core DV system (Applied Precision ${ }^{\mathrm{TM}}$, Issaquah, WA) equipped with an Olympus IX71 inverted microscope mounted with a Nikon Coolsnap ES2 HQ camera and a short arc 250W Xenon lamp (Advanced Light Microscopy Core Facility, Oregon Health and Science University, Portland, OR). Worms were immobilized immediately prior to imaging with a single drop of $5 \mathrm{M}$ levamisole (Vector Laboratories Inc., Burlingame, CA), a cholinergic agonist that prevents depolarization of muscle (Lewis et al. 1980). Images were optimized by deconvolving and relative ROS levels were quantified by manually enclosing the terminal pharyngeal bulb within each worm image to find the average intensity of the area using ImageJ software (NIH, Bethesda, MD). 
Working fluorescence measures were calculated as the difference between each stained sample and the mean non-stained controls of the same line imaged on the same day. Maximum values of MitoSOX fluorescence were used in all analyses because we previously determined that levamisole treatment had a significant effect on mean but not maximal MitoSOX fluorescence values (Hicks et al. 2012), and because maximum fluorescence measures offer greater consistency since they do not depend upon pixel size and/or the number/area of fluorescent signals within an image (Russ 2002). All images were acquired by the same person, and image analysis was performed in equal blocks by three researchers.

\section{Statistical analyses}

To assess the effects of strain and generation on line-specific nad5 $\Delta$ level, we carried out an ordinal logistic regression including the terms strain, generation, and the interaction strain $x$ generation. We also used chi-square tests to identify significant differences in nad5 $\Delta$ level between generational time points for individual strains. Because the number of MA lines per strain at $G_{F}$ varied between four and five (see above), randomly chosen MA lines from all strains were removed from analyses in order to balance the number of lines per strain at each generation (i.e., four lines per strain per generation). Comparisons involving $G_{0}$ and $G_{10}$ data and those involving only AF16 and ED-AF did not require the removal of lines. To assess the evolution of nad5 $\Delta$ over the course of the experiment, the percent of nad5 5 -containing mtDNA was calculated 
for each line as in Clark, et al (2012). These line-specific values were then used to calculate the average nad5 $\Delta$ percentage for each strain at each generation.

ROS measures were log transformed to achieve normality for the complete data set prior to analysis, but individual strains still displayed non-normal distribution and unequal variances following transformation. Non-parametric and parametric analyses produced the same results; we therefore report the parametric test results here. Also, we discovered statistically significant fluctuation across days of the assay in relative ROS level for the AF16 control line described above $(F=32.04, p<0.0001)$. We therefore subtracted the day-specific $G_{0}$ AF16 line 2 fluorescence measures (see above) from the scores for each sample prior to analysis. We analyzed ROS level data using a two-way ANOVA including the terms strain, generation, and the interaction strain $x$ generation. Separate ANOVAs were also performed to quantify variation in relative ROS levels among strains and among lines at each generational time point. Least-squares contrasts (Tukey's HSD for all pairwise comparisons) were used to test for differences between generational time points for every strain. We also calculated within- and among-line variance for each strain at $G_{0}, G_{10}$ and $G_{F}$.

To test for associations between ROS and nad5 deletion levels in C. briggsae, we conducted an ANOVA with nad5 $\Delta$ as the independent variable to determine if net ROS varied significantly according to line-specific nad5 levels. We also calculated Spearman's rank correlation coefficients between line-specific measures of ROS and 
nad5 $\Delta$ for individual strains at each generational time point. All statistical analysis was performed in JMP 9 (SAS Institute, Cary, NC).

\section{Results}

\section{$\underline{\text { Rates of MA line extinction }}$}

The C. briggsae strains studied here varied significantly in their measures of extinction risk under MA (ANOVA: $F=9085, p<0.0001$ ). As expected based on previous findings of low reproductive fitness in the high-deletion HK105 progenitor (Baer et al. 2005), MA lines initiated from this strain exhibited the fastest extinction times with all lines having gone extinct by generation 20. This was also true of the remaining HK105 MA lines that were not part of the current study (D. Denver, pers. comm.). MA lines of the hybrid ED-AF strain had the second-fastest extinction rates, followed by those generated from the remaining natural strains (Table 3.1). Although HK105 MA lines had the highest "rates of backup", the number of times a line was re-established from its

\begin{tabular}{lllll}
\hline Strain & Time to Extinction $(\mathrm{G})$ & $\mathrm{SD}$ & Backup Rate $\left(\# / \mathrm{G}_{\mathrm{F}}\right)$ & $\mathrm{SD}$ \\
\hline ED3101 & 48.80 & 1.643 & 0.0213 & 0.0301 \\
AF16 & 46.95 & 3.162 & 0.0458 & 0.0604 \\
HK105 & 17.51 & 2.510 & 0.1097 & 0.1555 \\
Hyb ED-AF & 38.39 & 1.817 & 0.0322 & 0.0307 \\
Total & 37.91 & 12.98 & 0.0522 & 0.0865 \\
\hline
\end{tabular}

Table 3.1. Time to extinction and backup rates of $\boldsymbol{C}$. elegans MA lines. The strain mean ( $n=5$ lines/strain) and grand mean ( $n=20$ lines) of time to extinction and backup rate. Time to extinction is defined as the number of MA generations undergone prior to declaring a line extinct. Backup rate was calculated as the ratio of the number of times a line was re-established from its backup population and the total number of MA generations the line experienced. SD is the standard deviation of each measure. 
backup population did not differ significantly among strains.

\section{Evolution of mitochondrial heteroplasmy}

To examine the effects of extreme bottlenecking on the evolution of nad5 $\Delta$ heteroplasmy levels within C. briggsae, we quantified nad5 $\Delta$ level in all MA lines at three generational time points: $\mathrm{G}_{0}, \mathrm{G}_{10}$, and $\mathrm{G}_{\mathrm{F}}$. At $\mathrm{G}_{0}$, HK105 had the highest nad5 levels at followed by AF16 (Fig. 3.2, Fig. 3.3A). As expected, neither ED3101 nor the EDAF hybrid (containing ED3101 mitochondria) acquired nad5 $\Delta$ over the course of the

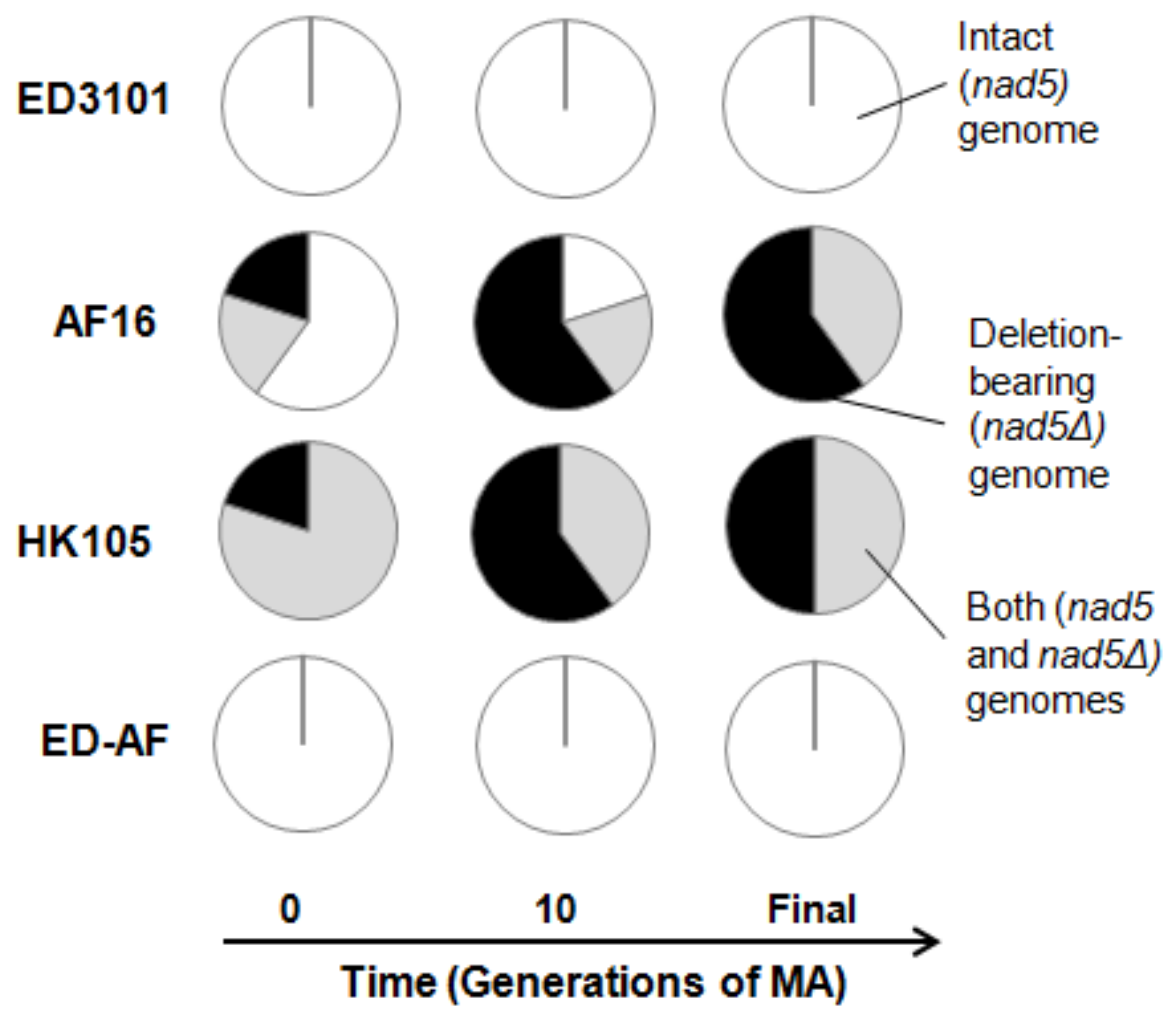

Figure 3.2 Evolution of nad5 $\Delta$ mtDNA levels under mutation accumulation treatment. Percentage of lines within each strain harboring either 1) all intact mitochondrial genomes (white portions), 2) some nad5 $\Delta$-bearing genomes and some intact genomes (gray portions), or 3) exclusively nad5 $\Delta$-bearing genomes as determined by a PCR-based method (black portions; see Materials and Methods and Howe \& Denver, 2008) at each generational time point. Note that the "Final" time point varied among strains; ED3101 and AF16 lines experienced 50 generations, ED-AF hybrid 40, and HK105 only 20. N=5 lines except where noted. 

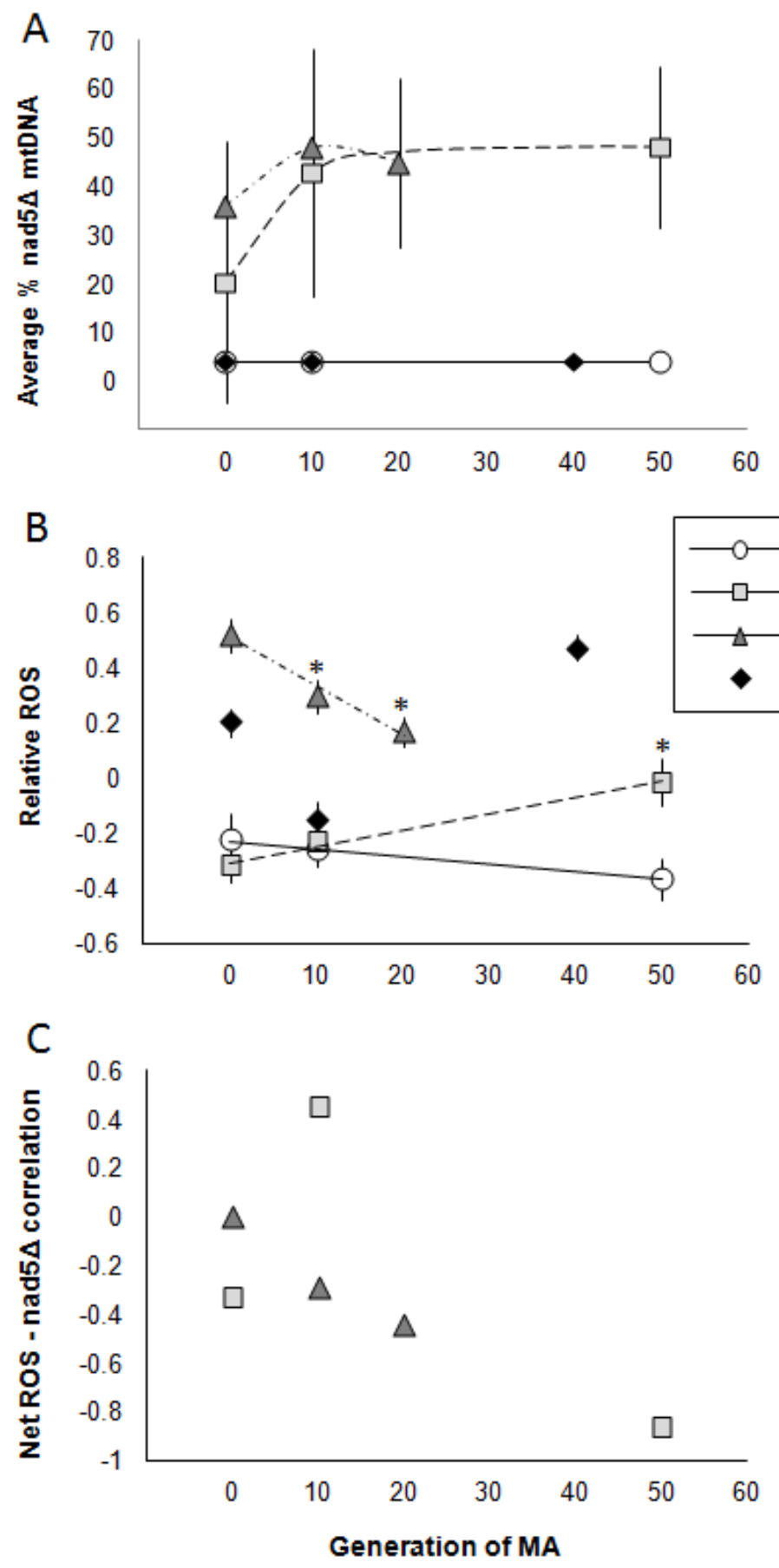

Figure 3.3. Evolution of average nad5 $\Delta$ level, relative ROS level, and the correlation between ROS and nad5 $\Delta$ levels over MA. A. Change in the average percentage of nad5 $\Delta$-bearing genomes. Asterisks indicates a significant difference from G0 in AF16 and HK105 (as determined by Tukey's HSD analysis). All measures of ED-AF hybrid were significantly different from each other. 
experiment, while AF16 and HK105 showed a general trend of increasing nad5 level with MA (Fig. 3.2, Fig. 3.3A). Accordingly, we observed a significant effect of strain on nad5 $\Delta$ heteroplasmy (Table 3.2), but no effect of generation on nad5 $\Delta$ level (Table 3.2). Removing both ED3101 and ED-AF - which lack the $\Psi$ nad5 -2 pseudogene and are unable to develop nad5 - from analysis resulted in a complete lack of fit for the model (data not shown). Additionally, we observed no significant changes in nad5 $\Delta$ level over the course of MA for either AF16 or HK105 individually (Table 3.3), although both AF16 and HK105 did experience statistically insignificant increases in nad5 $\Delta$ load (Figs. 3.2, 3.3A). Interestingly, the average percentage of nad5 $\Delta$-bearing mtDNA genomes within each of these strains increased to approximately $50 \%$ and then plateaued. Specifically, the (non-significant) increase in average percentage of nad5 $\Delta$ genomes from $G_{0}$ to $G_{10}$ was $110 \%$ in $\mathrm{AF} 16$ and $33 \%$ in $\mathrm{HK} 105$, with no comparable changes occurring from $\mathrm{G}_{10}$ to $\mathrm{G}_{\mathrm{F}}$ (AF16 increased $12 \%$ and HK105 decreased 6\%, Fig. 3.3A). Genotyping a larger number of lines may have revealed stronger effects of MA generation on nad5 $\Delta$.

\begin{tabular}{|c|c|c|c|c|}
\hline $\begin{array}{l}\text { Source of } \\
\text { Variation }\end{array}$ & DF & $x^{2}$ & $p$ & Evolution of mitochondrial ROS \\
\hline Strain & 3 & 49.65 & $<0.0001$ & measure the evolution of \\
\hline Generation & 2 & 7.784E-09 & 1 & $r h$ \\
\hline $\begin{array}{l}\text { Interaction } \\
\text { (Strain } x \text { Gen.) }\end{array}$ & 6 & 1.534 & 0.9572 & 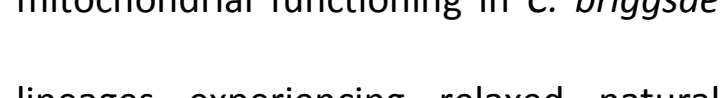 \\
\hline Whole Model & 11 & 52.69 & $<0.0001$ & latur \\
\hline
\end{tabular}

level for all MA lines at the same three generational time points. First, we found no evidence for an effect of researcher $(F=0.1338, p=0.7152)$ or for a researcher-by-strain 


\begin{tabular}{llllll}
\hline Strain & Gen & $\mathrm{N}$ & $\mathrm{DF}$ & $\mathrm{X}^{2}$ & $\mathrm{p}$ \\
\hline \multirow{4}{*}{ AF16 } & All gens & 15 & 4 & 6.083 & 0.1931 \\
& G0-G10 & 10 & 2 & 2.093 & 0.3512 \\
& G10-GF & 10 & 2 & 1.726 & 0.4219 \\
& G0-GF & 10 & 2 & 5.545 & 0.0625 \\
\hline \multirow{4}{*}{ HK105 } & All gens & 12 & 2 & 0.712 & 0.7006 \\
& G0-G10 & 10 & 1 & 1.726 & 0.1889 \\
& G10-GF & 8 & 1 & 0 & 1 \\
& G0-GF & 8 & 1 & 0.541 & 0.462 \\
\hline
\end{tabular}

Table 3.3. Chi-square results for strain-specific nad5 $\Delta$ differences between generational time points. interaction

effect $\quad(F=0.0446$, $p=0.9564)$ on final ROS measurements. Initial $\left(G_{0}\right)$ ROS levels for the three natural isolates studied here (Fig. 3.3B)

aligned well with those reported in Chapter 2 (Fig. 2.31). We observed no overall change in ROS level between $G_{0}$ and $G_{F}$ when analyzing the full dataset (Tukey HSD: Difference: 0.0472, $p=0.6060)$; however, there was considerable among-strain variation in the response of this trait to MA (Fig. 3.3B, Table 3.4). Along these lines, strain was the only variable to display significant effects on ROS variation (Table 3.4), although the model only described a minute amount of the total variation (adjusted $R^{2}=0.1398$ ). Remarkably, the evolution of strain-specific ROS level across generations of MA was highly linear for all C. briggsae natural isolates assayed here, a pattern often observed in traits related to fitness. By contrast, the hybrid ED-AF strain exhibited significant fluctuation in ROS levels across MA generations (Fig. 3.3B). The pattern of ROS evolution was, however, highly specific to each natural isolate: AF16 experienced significantly elevated ROS over MA, HK105 experienced a significant decline, and ED3101 exhibited no change in this trait throughout the entire 50-generation experiment. We also examined how variance in net ROS evolved over the course of MA. Based on standard 


\begin{tabular}{lccccc}
\hline $\begin{array}{l}\text { Source of } \\
\text { Variation }\end{array}$ & DF & $\begin{array}{c}\text { Sum of } \\
\text { Squares }\end{array}$ & $\begin{array}{c}\text { Mean } \\
\text { Square }\end{array}$ & F Ratio & Prob > F \\
\hline STRAIN & 3 & 3.147 & 1.049 & 4.366 & 0.0101 \\
GEN & 2 & 0.4192 & 0.2096 & 0.8723 & 0.4266 \\
GEN*STRAIN & 6 & 0.9118 & 0.1520 & 0.6325 & 0.7033 \\
Model & 11 & 4.478 & 0.4071 & 1.694 & 0.1145 \\
Within & 36 & 8.650 & 0.2403 & & \\
Total & 47 & 13.13 & & & \\
\hline
\end{tabular}

Table 3.4. Two-way ANOVA results for ROS variation among strains and generations. Effects of nad5 $\Delta$ and line(strain) decreased the model fit to the data (R2 decreased to 0.1081 and 0.0961 , respectively), and so were not included in the model.

expectations from MA experimental theory, we expected to observe increasing amongline variance in ROS levels with successive generations of MA. We observed significant among-line variation in ROS for all strains at nearly every generational time point (Table 3.5); however, AF16 was the only strain that followed the expected trend. Among-line variance in ROS for ED3101 declined while that for HK105 and ED-AF oscillated across generational time points (Fig. 3.4, Table 3.5).

\section{Possible relationships between $\operatorname{nad} 5 \Delta$ and ROS level}

As in previous studies (Estes et al. 2011; Hicks et al. 2012), we observed no relationship between line-specific ROS means and relative nad5 $\Delta$ level when considering the complete dataset (Spearman $\rho=0.1467, p=0.2718$ ); however, the association neared significance when comparing ROS and nad5 $\Delta$ from $\mathrm{G}_{0}$ and $\mathrm{G}_{10}$ only (Spearman $\rho=0.3082$, $p=0.0530$ ). Plotting these correlations at each generational time point revealed the strain-specific nature of the association between ROS and nad5 levels (Fig. 3.3C). The ROS-nad5 4 correlation became consistently weaker over MA in HK105, while that for AF16 exhibited extreme fluctuation. Because the Spearman correlation assigns ranks to 


\begin{tabular}{|c|c|c|c|c|c|c|c|}
\hline Strain & Generation & Source of Variation & df & SS & MS & $\mathbf{F}$ & $p$-value \\
\hline \multirow{9}{*}{ ED3101 } & \multirow{3}{*}{$\mathrm{G}_{0}$} & Line & 4 & 78.309 & 19.577 & 13.120 & $<.0001$ \\
\hline & & Within & 201 & 299.93 & 1.4922 & & \\
\hline & & Total & 205 & 378.24 & & & \\
\hline & \multirow{3}{*}{$\mathrm{G}_{10}$} & Line & 4 & 14.152 & 3.5381 & 5.8525 & 0.0002 \\
\hline & & Within & 197 & 119.09 & 0.6045 & & \\
\hline & & Total & 201 & 133.25 & & & \\
\hline & \multirow{3}{*}{$\mathrm{G}_{\mathrm{F}}$} & Line & 4 & 38.510 & 9.6276 & 9.9108 & $<.0001$ \\
\hline & & Within & 192 & 186.51 & 0.9714 & & \\
\hline & & Total & 196 & 225.02 & & & \\
\hline \multirow{9}{*}{ AF16 } & \multirow{3}{*}{$\mathrm{G}_{0}$} & Line & 4 & 10.907 & 2.7268 & 2.8710 & 0.0237 \\
\hline & & Within & 241 & 228.90 & 0.9498 & & \\
\hline & & Total & 245 & 239.80 & & & \\
\hline & \multirow{3}{*}{$\mathrm{G}_{10}$} & Line & 4 & 61.405 & 15.351 & 33.324 & $<.0001$ \\
\hline & & Within & 195 & 89.829 & 0.4607 & & \\
\hline & & Total & 199 & 151.23 & & & \\
\hline & \multirow{3}{*}{$\mathrm{G}_{\mathrm{F}}$} & Line & 4 & 107.46 & 26.865 & 27.211 & $<.0001$ \\
\hline & & Within & 193 & 190.55 & 0.9873 & & \\
\hline & & Total & 197 & 298.01 & & & \\
\hline \multirow{9}{*}{ HК105 } & \multirow{3}{*}{$\mathrm{G}_{0}$} & Line & 4 & 28.018 & 7.0045 & 11.393 & $<.0001$ \\
\hline & & Within & 188 & 115.58 & 0.6148 & & \\
\hline & & Total & 192 & 143.60 & & & \\
\hline & \multirow{3}{*}{$\mathrm{G}_{10}$} & Line & 4 & 6.4830 & 1.6208 & 2.0118 & 0.0942 \\
\hline & & Within & 199 & 160.32 & 0.8056 & & \\
\hline & & Total & 203 & 166.80 & & & \\
\hline & \multirow{3}{*}{$\mathrm{G}_{\mathrm{F}}$} & Line & 4 & 29.841 & 7.4603 & 16.641 & $<.0001$ \\
\hline & & Within & 192 & 86.074 & 0.4483 & & \\
\hline & & Total & 196 & 115.92 & & & \\
\hline \multirow{9}{*}{ ED-AF } & \multirow{3}{*}{$\mathrm{G}_{0}$} & Line & 4 & 16.810 & 4.2024 & 10.606 & $<.0001$ \\
\hline & & Within & 192 & 76.078 & 0.3962 & & \\
\hline & & Total & 196 & 92.888 & & & \\
\hline & \multirow{3}{*}{$\mathrm{G}_{10}$} & Line & 4 & 14.949 & 3.7372 & 4.1826 & 0.0029 \\
\hline & & Within & 185 & 165.30 & 0.8935 & & \\
\hline & & Total & 189 & 180.25 & & & \\
\hline & \multirow{3}{*}{$\mathrm{G}_{\mathrm{F}}$} & Line & 4 & 24.890 & 6.2225 & 20.303 & $<.0001$ \\
\hline & & Within & 192 & 58.845 & 0.3065 & & \\
\hline & & Total & 196 & 83.735 & & & \\
\hline
\end{tabular}

Table 3.5. One-way ANOVA results describing among-line variation in ROS level at each time point.

each data point based on the measured values, it was impossible to calculate for the 
ED3101 and the ED-AF hybrid lines due to their invariable nad5 levels. These strains were therefore excluded from the individual strain analysis. Further, an ANOVA analysis of the entire data set using line-specific nad5 $\Delta$ level as the independent variable
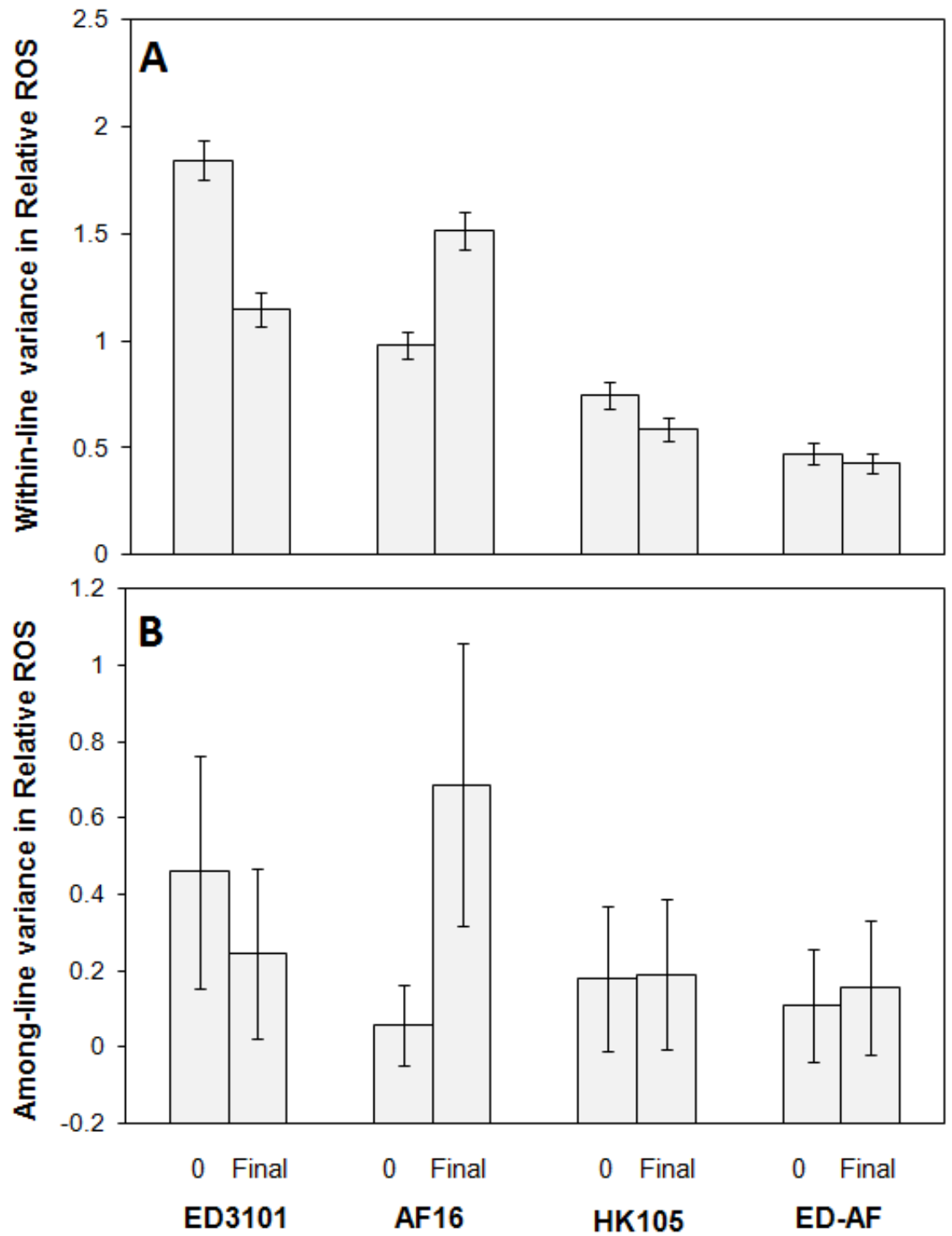

Figure 3.4. Variance in net ROS levels before and after 20-50 generations of extreme inbreeding. 0 indicates $G_{0}$ variance measures, while Final indicates the last generation reached by each line prior to extinction $\left(G_{F}\right) . G_{F}$ varied among strains and was $\sim 50$ generations for ED3101 and AF16 lines, 20 generations for HK105 lines, and $\sim 40$ generations for ED-AF lines. Bars indicate one standard error. A. Variance among all individuals (within-lines) of each strain $(N=193-246)$. B. Variance among lines of each strain $(\mathrm{N}=4-5)$. 
revealed that essentially none of the total variation in ROS level could be attributed to nad5 $\Delta$ level (ANOVA $F=2.456, p=0.0952, R^{2}=0.0820$ ). Although we observed no relationship between ROS and nad5 level, we observed a slight, non-significant increase in ROS in heteroplasmic lines (i.e., those that contained both intact and nad5 5 bearing genomes) (Tukey's HSD $p=0.2040$ ) - a trend that reached significance when the mitonuclear hybrid strain ED-AF was removed from analysis (Tukey's HSD $p=0.0426$ ). However, because we obtained only one measure of nad5 $\Delta$ for each line, this data must be interpreted with caution.

\section{Discussion}

We have used experimental evolution techniques with $C$. briggsae nematodes to conduct the first analysis of mitochondrial phenotypic and genotypic evolution under extreme genetic drift. Our study revealed a significant capacity for mitochondrial ROS evolution within C. briggsae maintained under standard laboratory mutationaccumulation treatment. Specifically, ROS levels in MA lines derived from C. briggsae natural isolates exhibited strikingly linear evolutionary trajectories, though the direction (increase or decrease) of evolution varied depending on strain (Fig. 3.3B). The mitonuclear hybrid strain displayed much more stochastic variation in ROS level over MA (Fig. 3.3B). The patterns of among-line variance in ROS level were also isolatespecific and did not support the hypotheses that ROS levels and among-line variance will consistently increase with successive generations of MA (Fig. 3.4, Table 3.5). Our data do 
support the idea that nad5 $\Delta$-bearing mitochondrial genomes act as selfish elements (Clark et al. 2012), demonstrated by the tendency for nad5 levels to increase with consecutive generations of MA (Figs. 3.2, 3.3A). We also found evidence suggesting that mitochondrial threshold effects may act on nad5 $\Delta$ heteroplasmy levels. Along these lines, our findings on population stability agree with previous studies indicating that high proportions of nad5 seem to carry negative consequences for nematode health and survival, while lower levels seem relatively benign (Howe and Denver 2008; Estes et al. 2011; Hicks et al. 2012). Therefore, the ability to maintain a reproductive population may be negatively associated with nad5 $\Delta$. Lastly, we observed no association between the patterns of evolution in ROS and nad5 $\Delta$ levels.

\section{Evolution of mitochondrial phenotypes under genetic drift}

MA studies consistently reveal a pattern of declining mean fitness and increasing among-line phenotypic variance (e.g. Mukai, 1964). Since elevated ROS levels are associated with mitochondrial dysfunction (Senoo-Matsuda et al. 2001; Wang et al. 2012), mean ROS levels might be expected to increase as a result of drift decay and in agreement with the vicious cycle theory (Bandy and Davison 1990). Contrary to this expectation, net ROS levels showed no general tendency to increase in response to mutation-accumulation treatment in C. briggsae (Fig. 3.3B). The highly linear evolution of mean ROS for the natural isolate lines mirrors patterns of evolution for fitness phenotypes (e.g., Baer et al. 2005); however, the direction of ROS evolution under MA was highly strain-specific and therefore in opposition to standard MA experimental 
predictions (Fig. 3.3B). A possible interpretation of this result is that net ROS level is differentially related to organismal fitness in these $C$. briggsae strains, although we believe a more plausible explanation is that patterns of ROS evolution will depend on the physiological state of the ancestral strain (see below). The hybrid ED-AF lines began the experiment with higher mean ROS levels than either of its parental lineages (ED3101 and AF16) (Fig. 3.3B). Because proper ETC function requires cooperation between the mitochondrial and nuclear genomes, this pattern may be symptomatic of disrupted mitonuclear epistases - expected if natural isolates ED3101 and AF16 have experienced functional divergence for ETC gene products. Unlike MA lines generated from the natural isolates, ED-AF exhibited a nonlinear pattern of change in ROS level across MA generations (Fig. 3.3B). Although further study is required to understand the basis for this difference, the nonlinear pattern of ROS evolution in ED-AF may be consistent with the further disruption of mitonuclear epistases by additional mutations presumed to have accumulated in these lines.

Similarly, we failed to detect a strong pattern of increase in among-line variance for mitochondrial ROS levels in C. briggsae, with only AF16 lines conforming to standard expectations for MA experiments (Fig. 3.4B). These results may owe themselves to the relatively short period of MA (i.e., small number of accumulated mutations) experienced by the lines in this study and/or to the unique biology of mitochondria. For instance, it is possible that we sampled HK105 and AF16 lines in different heteroplasmic states, and that this distinction underlies some of the ROS variation observed. However, we would 
expect to observe less within- and among-line variance for strains lacking the deletion if nad5 level is related to ROS (ED3101); this is not the case (Fig. 3.4). Further, it is unlikely that the accumulation of mutations within these lines is driving the straindependent patterns of ROS evolution. Rather, the unique physiology of each line, as determined by mitochondrial function and the genetic environment among other factors, likely underlies these strain-specific differences.

$\underline{\text { nad5 }} \Delta$ evolution and mitochondrial threshold effects

Despite the apparent negative consequences of nad5 $\Delta$ suggested by our results (Table 3.1) and others (Howe and Denver 2008; Estes et al. 2011), nad5 is known to persist in natural C. briggsae populations (Howe and Denver 2008) and in laboratory populations maintained at population sizes of $\sim 100$ or more (Estes, Coleman-Hulbert, Howe, and Denver, unpubl. data). Recent work indicating that nad5 $\Delta$-containing mitochondrial genomes act as a selfish element (Clark et al. 2012) may help to explain such findings. Our findings that nad5 $\Delta$ increased (albeit insignificantly) in both AF16 and HK105 lines maintained in the absence of selection (Figs. 3.2, 3.3A) supports this idea. Although sampling is limited, natural frequencies of nad5 levels have never been observed to accumulate to more than $60 \%$ of the total mtDNA population (Howe and Denver 2008). Similarly, Clark, et al. (2012) (which included more strains subjected to fewer generations of MA than the current study) also never witnessed nad5 $\Delta$ levels above $60 \%$ in bottlenecked lines. Finally, we observed an apparent plateau in the percentage of nad5 $\Delta$ genomes making up the total mtDNA population once they reach 
$\sim 50 \%$, a finding in line with previous work involving the heteroplasmic mtDNA deletion uaDf5 (Tsang and Lemire 2002). It is plausible that mitochondrial threshold effects may help to explain these patterns.

It has been proposed that a mitochondrial threshold effect, wherein a certain fraction of mutant mitochondrial genomes is required to generate deleterious phenotypes (Rossignol et al. 2003), may partially explain many counterintuitive results comparing mitochondrial ROS, mutation rates, and genetic damage (Ventura et al. 2006; Gruber et al. 2011). Based on the idea of a "vicious cycle" between ROS levels and mutation rates (Bandy and Davison 1990; Kowald and Kirkwood 1996), we expected to observe a statistical association between ROS and generation of MA; no such relationship emerged (Fig. 3.3B). Previous work reporting elevated ROS levels (Estes et al. 2011; Hicks et al. 2012), reduced fecundity (Howe and Denver 2008; Estes et al. 2011), and altered mitochondrial morphology (Hicks et al. 2012) in HK105 relative to other C. briggsae isolates suggests that HK105 may have reached a threshold for nad54bearing genomes, such that it suffers the deleterious consequences of mitochondrial dysfunction. Along these lines, we also observed an apparent threshold for nad5 $\Delta$ level within HK105 and AF16 (the two lines able to accumulate the deletion). Competing influences of a replicative advantage for nad5 5 genomes and selection against them at high levels may establish an upper-bound for nad5 $\Delta$ heteroplasmy level within $C$. briggsae, above which severe dysfunction ensues. The accumulation of mutations during the course of our study may have further reduced HK105 mitochondrial function, 
resulting in declining ROS levels due to diminished overall mitochondrial capacity. This threshold idea is further bolstered by the observation that ROS levels were greatest in lines with the highest incidence of heteroplasmy, i.e., when the mitochondrial population harbored both nad5 $\Delta$-bearing and intact genomes. Work in human cybrid cells carrying a frameshift mutation in ND5 revealed a similar trend, with the heteroplasmic cell line experiencing slightly elevated ROS compared to both the homoplasmic wild-type and the nearly-homoplasmic mutant cell lines (Park et al. 2009), that was attributed to severely reduced mitochondrial function in the nearlyhomoplasmic mutant cell lines (Park et al. 2009). Overall, the strain-specific relationships between ROS, MA generation and nad5 $\Delta$ level suggest a role for mitochondrial threshold effects in C. briggsae and are consistent with other work showing that ROS levels will not necessarily correlate to mutation accumulation (JoynerMatos et al. 2011) or other types of genetic damage (Ventura et al. 2006). However, our small sample size for the genotyping portion of this assay and the lack of mutation rate estimates limits further interpretation of this data.

\section{Conclusions and outlook}

We have reported the first application of experimental mutation-accumulation to examine a mitochondrial phenotypic response to extreme genetic drift, as well as an analysis of the evolution of heteroplasmy of a large mitochondrial deletion (nad5 $\Delta$ ) in $C$. briggsae. We found that average levels of both ROS and nad5 heteroplasmy evolved in a highly linear fashion in MA lines generated from three natural isolates. nad5 $\Delta$ 
heteroplasmy level was found to plateau after reaching $\sim 50 \%$ in isolates vulnerable to the deletion. However, the direction of mitochondrial ROS evolution was found to be strain-specific, leading to strain-specific relationships between ROS and nad5L, and ROS and generation of MA. Our results align well with a detrimental effect of nad5 $\Delta$ at high levels, demonstrated by the extinction of all lines generated from the high-deletion isolate HK105 prior to the twentieth generation of MA. Finally, the well-established pattern of increasing among-line variance expected for mutation accumulation experiments was not generally supported by our data. Although our work represents a step forward in our comprehension of the evolutionary forces potentially influencing mitochondrial ROS and heteroplasmy levels, additional work is required to fully understand the genetic and developmental mechanisms governing the relative strengths of these evolutionary forces on mtDNA and its associated traits. Further advancement toward this end will require more thorough genotyping, including wholegenome sequencing combined with assessments of fitness and other important phenotypes across several generations.

While our study highlights the potential for MA experiments to advance our understanding of mitochondrial evolution, several fundamental questions remain unanswered. We have yet to determine how and why certain mtDNA molecules are inherited. Further, experimental methods for manipulating the size of the mitochondrial bottleneck or the strength of selection on mtDNA have yet to be developed. We now know that a complex mitochondrial life cycle involving organelle fission, fusion and 
autophagy may act to purge damaged mtDNA genomes (Twig et al. 2008b; Kuznetsov and Margreiter 2009; Kowald and Kirkwood 2011; Bess et al. 2012; Meyer and Bess 2012), yet we know essentially nothing about the evolutionary or cell-biological factors that may influence its efficacy. Because $C$. briggsae is amenable to experimental evolution and cell biological study, and harbors significant mitochondrial genetic and phenotypic variability, it will be a valuable tool for future research in this arena. 


\section{CHAPTER FOUR}

Reactive oxygen species level is related to 8-oxo-dG content but not germline base substitution rate in Caenorhbaditis elegans

Kiley A. Hicks, Joanna Joyner-Matos

\section{Introduction}

Although reactive oxygen species (ROS) are important for cell signaling and the regulation of apoptosis (Tuma 2001), excessive levels of ROS within eukaryotic cells have been linked to the accumulation of somatic nuclear mutations in several human diseases (Klaunig and Kamendulis 2004; Wallace 2005a). However, whether ROS play a major role in generating heritable germline mutations is less clear (Stoltzfus 2008; Joyner-Matos et al. 2011). Within eukaryotes, mitochondria are the primary source of endogenous ROS, which are generated as a byproduct of oxidative phosphorylation at the electron transport chain (ETC). Healthy mitochondria produce ROS in sufficiently small amounts that excess ROS may be quenched by cellular antioxidants before damaging nucleic acids, proteins or lipids (Sedensky and Morgan 2006; Imlay 2008). However, ETC dysfunction and impairment can increase ROS generation (Verkaart et al. 2007; Chen et al. 2008; Dingley et al. 2009) and lead to imbalances between the oxidant load and antioxidant capacity within a cell (Grad and Lemire 2004) - a condition known as oxidative stress (Halliwell and Gutteridge 2007). Oxidative DNA damage has been 
implicated as a main contributor to decreased cellular function in sperm cells following oxidative stress (Aitken 1995; Lewis and Aitken 2005; Aitken et al. 2009), and both oxidative stress and germline DNA damage is implicated in the evolution of aging (Medawar 1952; Kirkwood 2005) and life-history traits (Velando et al. 2008; Dowling and Simmons 2009).

Given the importance of genetic fidelity to organismal health and fitness, eukaryotic cells rely upon various repair mechanisms to prevent permanent DNA damage (Croteau 1997; Lu et al. 2001; Pascucci et al. 2011; Berquist and Wilson 2012). In particular, a great deal of research effort has focused on the oxidized guanine product 8-oxo-dG because its unique biochemistry makes it pro-mutagenic (David et al. 2007; Valavanidis et al. 2009). 8-oxo-dG mimics thymine biochemically making replicative DNA polymerases less effective at recognizing these lesions as damaged bases (Shibutani et al. 1991; Hsu et al. 2004). If replication proceeds without repair, the oxidized base is converted into thymine, ultimately resulting in a G-to-T transversion mutation at the site of the lesion (Cheng et al. 1992; Kasai 2002; David et al. 2007). Thus, the presence of 8oxo-dG and G-to-T mutations has been considered a hallmark of oxidative stress (Busuttil et al. 2005). While some evidence supports the idea that oxidative stress leads to an increased frequency of G-to-T mutations (Hussain et al. 2000; Busuttil et al. 2003), other work suggests a more complicated relationship between oxidative stress and genetic damage. For example, G-to-T base substitutions have been linked to oxidative stress in liver but not in brain tissues in the mouse (Busuttil et al. 2005). Much of this 
work has been conducted on somatic tissues in flies, mice, and cell culture (Dolle et al. 2000; Vijg 2000; Garcia et al. 2007). Because the mutational processes of the soma and germline may be distinct, additional studies of germline mutation are needed (Drake et al. 1998; Fortune 2000; Martorell et al. 2000; Shanks et al. 2008; Crabbe and Hill 2010; Lynch 2010; Joyner-Matos et al. 2011).

Because the study of heritable mutation requires multi-generation experiments, there have been more examinations of the relationship between oxidative stress and somatic mutation as opposed to heritable germline mutation. Caenorhabditid nematodes have become an important model system for studying the cellular and genetic underpinnings of the oxidative stress response (Grad and Lemire 2004; Kayser et al. 2004; An et al. 2005; Inoue et al. 2005; Lee et al. 2010; Yasuda et al. 2011) and have proven to be valuable for multi-generation evolutionary studies (Denver et al. 2000, 2006; Ajie et al. 2005; Baer et al. 2005; Estes et al. 2005). Joyner-Matos, et al. (2011) examined fitness degradation as a proxy for germline mutation accumulation in two sets of long-term mutation-accumulation (MA) lines: one set derived from canonical $C$. elegans lab strain (N2), and the other from an ETC Complex II mutant (mev-1) which is predicted to experience elevated ROS levels (Senoo-Matsuda et al. 2001). Worms were maintained in population sizes of one to allow mutations to accumulate under relaxed selection for $\sim 125$ generations. Because the majority of new mutations causing phenotypic effects have negative consequences for organismal fitness, mean fitness is expected to experience an approximately linear decline over successive generations of 
this treatment (e.g., Fig. 5 in Mukai 1964). Because they observed no difference in the mutational decline in fitness between the two sets of MA lines, Joyner-Matos, et al. (2011) concluded that oxidative stress did not contribute significantly to germline mutation accumulation. However, this was not confirmed directly through genetic analysis (Joyner-Matos et al. 2011). Denver, et al. (2006) used four sets of MA lines generated from repair-deficient mutants (two mismatch repair mutants, one base excision repair mutant and one nucleotide excision repair mutant) combined with an identical experimental approach to directly estimate germline mutation rates in $C$. elegans. MA lines derived from the repair-deficient mutants experienced significantly elevated base-substitution rates and, in most cases, elevated rates of insertion-deletion mutations as compared to those generated from N2 (Denver et al. 2006). Importantly, all three of the repair pathways tested have been implicated in the repair of oxidative damage (Denver et al. 2006; Fensgård et al. 2010), suggesting that oxidative stress may elevate germline mutation rates. A separate study by Denver, et al. (2009) revealed high rates of G-to-T mutations within the nuclear genome of a set of long-term ( 250 generations) MA lines of $C$. elegans generated by (Baer et al. 2005). This finding, together with recent evidence for significant variation in relative mitochondrial ROS levels among natural and experimental strains of Caenorhabditids (Dingley, et al., 2009; Estes, et al., 2011; and see Figs. 2.3I, 3.3B), suggests that oxidative stress may contribute to heritable mutation accumulation in $C$. elegans. These results highlight a need for a direct comparison of ROS, oxidative DNA damage and germline mutation rates. 
We assayed ROS levels and 8-oxo-dG content in five long-term mutation accumulation lines and their wildtype progenitor line from the study by Baer et al. (2005), all of which had been previously subjected to whole-genome sequencing (Denver et al. 2009), in order to study the relationship between relative ROS, oxidative DNA damage and germline mutation rates in C. elegans. Based on theory and previous work (Cheng et al. 1992; Hussain et al. 2000; Busuttil et al. 2003, 2005; David et al. 2007), we expected MA lines to score higher in indicators of oxidative stress - higher ROS and 8-oxo-dG content - and to experience elevated rates of G-to-T base substitutions relative to the N2 progenitor, resulting in linear relationships between our oxidative stress measures and previously reported nuclear mutation rates. Our expectations were only partially borne out by the data. While the $\mathrm{N} 2$ progenitor line consistently displayed relatively low levels of ROS and 8-oxo-dG compared to the MA lines, only two MA lines exhibited significantly elevated ROS levels, and no significant differences among lines in 8-oxo-dG content were revealed. Despite this inconsistency, we observed a strong, positive correlation between steady-state ROS levels and total 8oxo-dG content. Contrarily, no obvious relationships between both oxidative stress measures and the G-to-T mutation rate data emerged. Our results support the contention that G-to-T mutations may not reliably manifest the extent of heritable oxidative damage to nuclear DNA in C. elegans.

\section{Materials and Methods}




\section{Nematode strains and culture conditions}

For this study, we used a set of five $C$. elegans mutation-accumulation lines and their common ancestral strain that were previously subjected to whole-genome sequence analysis (Denver et al. 2009). The MA lines were part of a larger group of 100 long-term MA lines that were generated from the self-progeny of an inbred Bristol N2 nematode and maintained as described in Baer et al. (2005). Assays were performed using worm stocks that were cryogenically preserved using standard freezing protocols (Stiernagle 2006) following 250 generations of MA. Prior to ROS level assays, frozen stocks for each MA line and the N2 progenitor were thawed and worms were allowed to recover for one week under standard laboratory conditions at $20^{\circ} \mathrm{C}$ with regular population transfers to fresh $15 \mathrm{~mm}$ NGM Petri plates seeded with OP50 Escherichia coli.

Two separate bleaching protocols (Stiernagle 2006) were then performed to yield two independent, age-synchronous populations of each line; half of each population was reserved to create line-specific internal control groups. Each experimental population was then analyzed for ROS level as outlined below. For analysis of 8-oxo-dG levels, five individuals from each line were carried through three generations of single-individual descent at four day intervals. Each replicate was subsequently allowed to generate a large population which was age-synchronized. Upon reaching the L4 larval stage, each age-synchronous population was transferred to plates containing $40 \mu \mathrm{M}$ 5-fluoro-2'-deoxyuridine (FudR, which prevents progeny production). 
Because oxidative damage is more successfully detected in older nematodes (Adachi et al. 1998; Yasuda et al. 1999) assays were performed on 12-day-old nematodes that had been washed in $\mathrm{M9}$, flash-frozen with liquid $\mathrm{N}_{2}$ and stored at $-80^{\circ} \mathrm{C}$ until analysis.

\section{Fluorescence microscopy}

To study the evolution of mitochondrial ROS levels under MA, we performed confocal image analysis on live young adult nematodes as described previously (Dingley et al. 2009; Estes et al. 2011; Hicks et al. 2012). In brief, worms were incubated for 24 hours at 20C in the presence of 10uM MitoSOX Red (Molecular Probes Inc., Eugene, OR) - a mitochondria-targeted fluorescent dye that measures total levels of mitochondrial oxidants (Zielonka and Kalyanaraman 2010). Control worms were incubated concurrently under identical conditions, replacing MitoSOX with water.

For each MA line, fluorescent z-stack images of the pharyngeal bulb of 15-20 treatment and 5 control worms were photographed at 60X magnification. Images were acquired using an Olympus IX71 inverted microscope mounted with a Nikon Coolsnap ES2 HQ camera and a short arc $250 \mathrm{~W}$ Xenon lamp, all part of a high resolution wide field Core DV system (Applied Precision ${ }^{\mathrm{TM}}$, Issaquah, WA) (Oregon Health and Sciences University Advanced Light Microscopy Core Facility, Portland, OR). Worms were exposed to a cholinergic agonist that prevents depolarization of body-wall muscle (Lewis et al. 1980) immediately prior to imaging, and abracadabra, immobilized. Deconvolutionoptimized images were used to quantify relative ROS levels by manually enclosing the terminal pharyngeal bulb within each image and obtaining the average intensity of the 
area using ImageJ software (NIH, Bethesda, MD) as described in (Dingley et al. 2009). All images were acquired and analyzed by $\mathrm{KAH}$.

Previous work with fluorescence microscopy has demonstrated that maximum fluorescence measures offer greater consistency as they do not depend upon pixel size or on the number/area of fluorescent signals within an image (Russ 2002). Additionally, we previously determined that levamisole treatment does not affect maximum MitoSOX fluorescence but does significantly alter mean MitoSOX fluorescence values (Hicks et al. 2012). Thus, maximum values of MitoSOX fluorescence were used for all statistical analyses.

\section{DNA extraction for 8-oxo-dG assays}

To minimize oxidation during sample preparation (Helbock et al. 1998) we used the chaotropic sodium iodide method of DNA extraction (Ishizawa et al. 1991) using a Wako DNA Extractor TIS kit (Wako, Osaka, Japan) and following the manufacturer's protocol, but with an overnight incubation at $-80^{\circ} \mathrm{C}$ (in $70 \%$ ethanol) and a lengthened RNase step. A Qubit Fluorometer (Life Technologies, Grand Island, NY) combined with RNA Assay, dsDNA HS Assay and Protein Assay kits (Life Technologies, Grand Island, NY) were used to quantify RNA, DNA, and protein content. RNA and protein were undetectable in all but one sample, which had slightly elevated RNA levels and was discarded due to the specificity of the assay for both 8-oxodG and 8-oxoG. Samples were diluted to a range of 0.05 to $0.2 \mathrm{ng} / \mu \mathrm{L}$ of DNA prior to ELISA analysis. A standard curve was generated by diluting an oligonucleotide stock solution containing 8-oxo-dG 
(Trevigen, Gaithersburg, MD) to 1:500, 1:750, and 1:1000 in TE/oxidation inhibitor buffer.

\section{8-oxodG Enzyme-Linked ImmunoSorbent Assay (ELISA)}

To quantify 8-oxodG content, samples, standards and a negative control (TE/oxidation inhibitor buffer) were incubated with intermittent vortexing for 10 minutes with an equal volume of Reacti-Bind (Pierce DNA coating solution, Thermo Scientific, Rockford, IL). $100 \mu \mathrm{L}$ of each sample was loaded into triplicate wells arranged randomly across three Nunc MaxiSorp 96-well plates, leaving one set of wells on all four sides of each plate empty to minimize sample dehydration. Plates were incubated overnight at room temperature on an orbital shaker.

The next day, wells were washed three times with phosphate buffered saline with $0.05 \%(v / v)$ Tween-20 (PBS-t). Wells were then subjected to three sequential incubation steps at $37^{\circ} \mathrm{C}$ with shaking and PBS-t washes between each step: 1) one hour in blocking solution (200 $\mu \mathrm{L}$ of $0.5 \%(v / v)$ fetal calf serum in PBS-t), 2$)$ two hours with the primary antibody (anti-8-oxodG, Clone 2E2, Trevigen), and 3) two hours with of secondary antibody (goat anti-mouse IgG, alkaline phosphatase conjugated). Wells were then washed before the addition of the $\mathrm{p}$-Nitrophenylphosphate Alkaline Phosphatase Substrate solution (Vector Laboratories, Burlingame, CA) and incubation in the dark at room temperature for up to three hours. Absorbance was measured every 30 minutes for three hours at $405 \mathrm{~nm}$ wavelength. The signal increased in intensity until 2.5 hours, 
and then did not change between 2.5 and 3 hours. The data from the 2.5 hour read are presented here.

Data for each sample and standard was corrected by subtracting the average optical density of three blank wells specific to each plate. The corrected optical densities for the 8-oxo-dG standard curves on each plate were modeled by the one-site saturation, ligand-binding curve fit in SigmaPlot 11 (Systat Software, Inc., San Jose, CA) and the resulting regression equation was used to calculate the nanograms of DNA equivalents per well. Finally, we used the copy number template from the URI Genomics and Sequencing Center (http://www.uri.edu/research/gsc/resources/cndna.html) to calculate the number of damaged bases per well, and we report the data $\times 10^{6}$ damaged bases per nanogram of DNA.

\section{Calculation of Mutation Rate}

The per generation rate of base substitution mutations $\left(\mu_{\mathrm{BS}}\right)$ was calculated for each line following (Denver et al. 2009) by dividing the number of new base substitutions that arose during MA by the product of the total number of nucleotides sequenced (reported in Denver et al., 2009) and the approximate number of generations experienced by each MA line (D. Denver, pers. communication). The per generation rate of G-to-T transversions $\left(\mu_{\mathrm{G}-\text { to-T }}\right.$ ) was calculated by dividing the number of G-to-T mutations by the product of the number of $\mathrm{G} \cdot \mathrm{C}$ sites considered and the estimated number of generations experienced by each MA line.

\section{$\underline{\text { Statistical analyses }}$}


Because our ROS and 8-oxo-dG data violated the assumptions of equal variance and normal distribution, we used the Kruskal-Wallis rank sums test to examine amongstrain differences in ROS and oxidative damage and a Wilcoxon method to evaluate differences among pairs of lines. We also calculated Spearman's rank correlation coefficients to evaluate the relationships between ROS levels and 8-oxo-dG content, as well as between these traits and the overall rate of nuclear base-substitutions and G-toT transversions. Because ROS level, 8-oxo-dG content and base substitution rates were measured on different sets of worms, correlation analyses were conducted using linespecific averages for each character. All analyses were performed using JMP 9 (SAS Institute, Cary, NC).

\section{Results}

We analyzed net ROS and 8-oxo-dG levels with the aim of quantifying variation in the amount of oxidative stress experienced by $C$. elegans MA lines. First, all MA lines assayed had higher in vivo ROS levels compared to their N2 ancestral strain (see Materials and Methods), but the increase was statistically significant for only two lines (523 and 574) (Fig. 4.1). Interestingly, the line with the highest ROS level (523) also exhibited the highest within-line variance (as determined by calculations of standard error) for ROS (Table 4.1). Second, mean 8-oxo-dG content did not differ significantly 
among MA lines (Kruskal-Wallis $\mathrm{H}=8.396, \mathrm{p}=0.136$ ); however, these data exhibited a

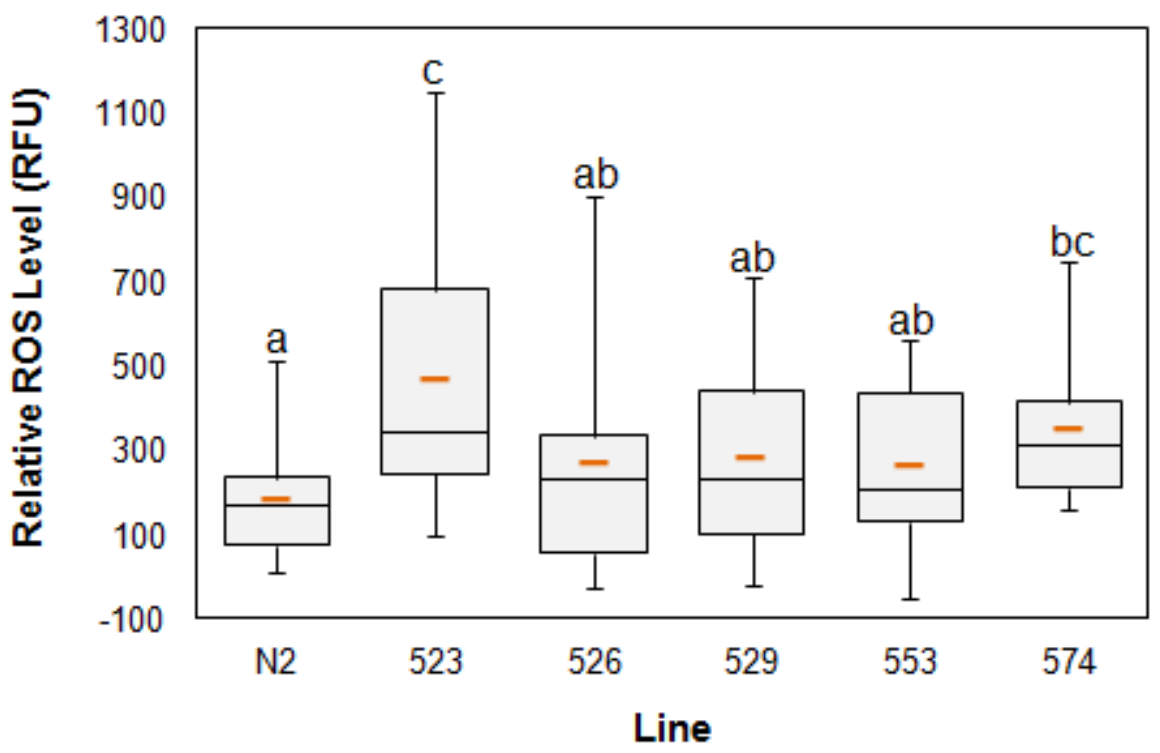

Figure 4.1 - Quantification of relative in vivo ROS levels in C. elegans terminal pharyngeal bulbs. Net oxidant levels in young adult nematodes were assessed using the average maximum pharyngeal bulb fluorescence for 15-20 worms for each mutation-accumulation line. Relative ROS levels differed significantly among lines (Kruskal-Wallis: $X^{2}=13.35, p=0.0203$ ), but only 523 and 574 exhibited increased ROS levels relative to N2 (Wilcoxon each pair: $523 p=0.0009$; $574 p=0.0028$ ). Box length represents 25 th-75th percentile inter-quartile range, interior horizontal line represents median, vertical lines issuing from the box extend to minimum and maximum values, orange dash identifies the mean. Letters above capped lines denote significantly different groups as determined by Wilcoxon each pair test.

similar trend as those for ROS with the N2 progenitor line exhibiting the lowest levels relative to all MA lines (Table 4.1).

We next analyzed the relationships between the two measured oxidative stress phenotypes, and between these characters and previously reported per-generation base substitution rates for the same $C$. elegans lines (Denver et al. 2009). First, linespecific ROS levels were highly positively correlated with line-specific 8-oxo-dG content 


\begin{tabular}{ccccccc}
\hline Line & Relative ROS & SE of ROS & 8-oxo-dG & $\begin{array}{l}\text { SE of 8- } \\
\text { oxo-dG }\end{array}$ & $\mu_{\text {BS }}$ & $\mu_{\text {G-TO-T }}$ \\
\hline 523 & 471.36 & 75.800 & 54921 & 7400.3 & $3.163 \mathrm{E}-09$ & $3.987 \mathrm{E}-05$ \\
526 & 269.19 & 57.333 & 35950 & 4648.9 & $2.446 \mathrm{E}-09$ & $1.417 \mathrm{E}-05$ \\
529 & 282.78 & 49.182 & 33720 & 1111.9 & $1.845 \mathrm{E}-09$ & $2.080 \mathrm{E}-05$ \\
553 & 261.52 & 48.611 & 26435 & 7769.7 & $2.890 \mathrm{E}-09$ & $3.687 \mathrm{E}-05$ \\
574 & 350.39 & 38.381 & 50792 & 10438 & $1.757 \mathrm{E}-09$ & $1.982 \mathrm{E}-05$ \\
N2 progenitor & 192.97 & 29.577 & 23971 & 8736.2 & - & - \\
\hline
\end{tabular}

Table 4.1. Variation in Oxidative Stress-Related Traits. Means and standard errors (SE) of net in vivo ROS levels $(n=15-20)$ and 8-oxo-dG content $(n=3)$ in five $C$. elegans mutation-accumulation lines and their progenitor strain. The per generation rate of base substitution mutations ( $\mu \mathrm{BS})$ and G-to-T transversion mutations ( $\mu$ G-TO-T) per site was calculated following Denver, et al. (2009) and further described in Materials and Methods. The N2 progenitor line was not included in the Denver et al., (2009) study, thus estimates of mutation rates could not be made

(Spearman's $\rho=0.943, p<0.05 ;$ Fig. 4.2). However, we detected no relationship between ROS level and either the overall base substitution rate (Spearman's $\rho=0.000, p=1.000$ ) or the G-to-T transversion rate (Spearman's $\rho=0.300, p=0.624$ ) reported in Denver, et al (2009). Similarly, we found no association between 8-oxo-dG levels and either measure of mutation rate ( $\mu_{\mathrm{BS}}$ : Spearman's $\rho=0.100, \mathrm{p}=0.873 ; \mu_{\mathrm{G}-\text { to-T }}$ Spearman's $\rho$ $=0.100, p=0.873)$ for the lines assayed. 


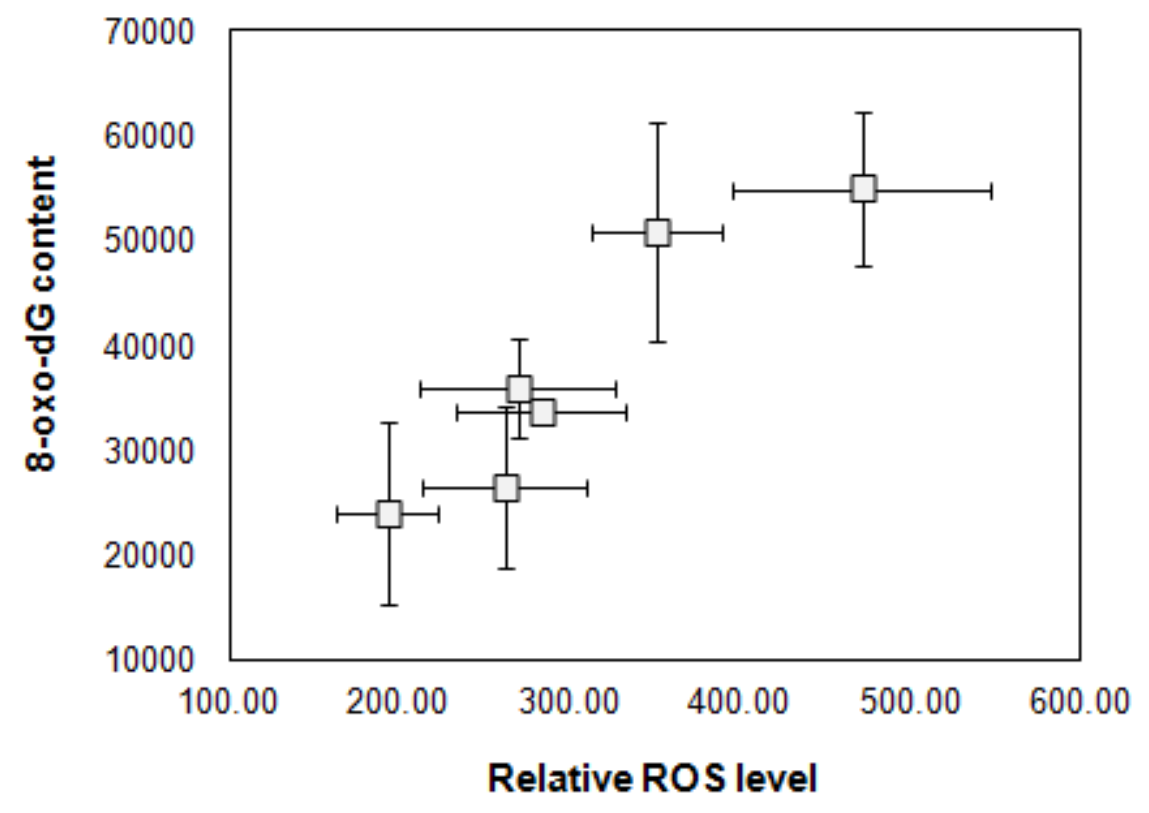

Figure 4.2. Bivariate relationship of MA line means for net in vivo ROS level and 8-oxo-dG content. Spearman's $\rho=0.943, p<0.05$. Bars represent one standard error.

\section{Discussion}

We quantified total 8-oxo-dG content and in vivo mitochondrial ROS levels in mutation-accumulation lines of $C$. elegans that had previously undergone wholegenome sequencing with the aim of clarifying the relationships between oxidative stress and heritable base substitution mutations in C. elegans. Our results revealed significant variation in net ROS levels among lines that had undergone approximately 250 generations of mutation-accumulation (Fig. 4.1), but no such variation in 8-oxo-dG content. Still, a robust, positive relationship between steady-state ROS levels and total 
8-oxo-dG content emerged (Fig. 4.2). Finally, we observed no obvious correlations between both oxidative stress measures and either $\mu_{B S}$ or $\mu_{G-\text {-to- }}$ in the same MA lines.

Several recent studies have revealed significant variation among natural isolates and laboratory mutants of Caenorhabditid worms in steady-state ROS levels (Dingley et al. 2009; Estes et al. 2011; Hicks et al. 2012). Thus, our finding that relative ROS levels were significantly elevated above the progenitor line in two MA lines are not unexpected. It is noteworthy that $C$. elegans displays greater variance in ROS among the MA lines assayed here than among laboratory mutants with defects of the mitochondrial ETC (Fig. 4.1, and see Fig. 2 in Dingley et al., 2009). This distinction may be partially explained by differences in experimental procedure that arbitrarily inflated the observed within-strain variation in the ETC mutants (i.e., ROS measures made on separate days in the ETC mutant assay). Further, we uncovered no significant amongline variation in 8-oxo-dG level, a finding that agrees with results from the Denver, et al. (2009) study revealing a no significant variation in base substitution rates in these lines.

A remarkably strong correlation emerged between relative ROS levels and 8-oxodG content as measured here (Fig. 4.2). This result is aligns well with previous experiments (Kasai and Nishimura 1984, 1986; Floyd et al. 1988; Beehler et al. 1992) but is particularly meaningful given our small sample size. Conversely, our findings failed to identify any relationship between the two oxidative stress phenotypes assayed and previously determined nuclear genome base substitution rates (Denver et al. 2009). A good deal of theoretical and experimental research predicts a statistical association 
between G-to-T mutation rates and measures of oxidative stress (Cheng et al. 1992; Hussain et al. 2000; Busuttil et al. 2003, 2005; David et al. 2007). C. elegans might be particularly susceptible to oxidatively induced G-to-T substitutions because they possess a very limited base excision repair system, lacking any DNA glycosylases specific for oxoguanine, formamidopyrimidine, and methyladenine (Denver et al. 2003b, 2006). Thus, an oxidized guanine base should more often result in a G-to-T transversion. However, recent work suggests that the accumulation of G-to-T mutations may not consistently relate to ROS levels, but that large genomic rearrangements could be important signals of oxidative stress, especially in ectotherms (Dolle et al. 2000; Vijg and Dollé 2002; Hasty et al. 2003; Busuttil et al. 2007; Crabbe and Hill 2010; Garcia et al. 2010; Joyner-Matos et al. 2011). Using a lacZ reporter gene assay, Garcia, et al. (2010) observed a higher mutation frequency in the somatic tissue of flies compared to mice, with a much larger fraction being genome rearrangements. Culturing the flies at warmer temperatures further increased the rates of genome rearrangements within highly oxidative somatic tissues, leading the researchers to propose that increased respiration and ROS production rates induced by elevated temperatures may explain the observed trend (Garcia et al. 2010). Busuttil, et al. (2007) showed that although G-to-T mutations were prevalent in mitotically-active tissues, genome rearrangements were more common in quiescent cells. These results combined with the distinct mutational processes of the soma and germline (Drake et al. 1998; Fortune 2000; Martorell et al. 2000; Shanks et al. 2008; Crabbe and Hill 2010; Lynch 2010; Joyner-Matos et al. 2011), 
and the fact that both base substitutions and large genome rearrangements may be attributable to ROS damage, suggest that different mutational types may associate with oxidative stress depending on the tissue or organism in question. Alternatively, redundancy in repair mechanisms (Barnes and Lindahl 2004; Arczewska et al. 2008; Fensgård et al. 2010) may prevent the accumulation of oxidative genetic damage precluding a direct association between oxidative stress measures and heritable mutation.

One caveat that prevents further interpretation of the above correlations is that the mutation rate data used in our comparisons was derived from the nuclear genome. While theoretical and empirical evidence suggests that mitochondrial ROS can damage cytoplasmic and nuclear components including nuclear DNA (Richter et al. 1988; Winterbourn 2008), there is evidence that the mitochondrial genome sustains much more oxidation-induced damage (Richter et al. 1988; Yakes and Van Houten 1997; Shokolenko et al. 2009), and thus may more accurately describe the relationship between heritable mutation and oxidative stress. Additionally, mounting evidence implies that the relationship between mitochondrial ROS and nuclear genetic damage may be indirect. For example, mitochondrial impairment may reduce repair efficiency in the nuclear genome (Delsite et al. 2003). Further, recent work has uncovered a ROS response to genetic damage (Rowe et al. 2008), which in turn may act to regulate base excision repair (Swartzlander et al. 2010) and the Yap1 transcription factor (Rowe et al. 2012) to maintain genetic fidelity in Saccharomyces cerevisiae. Thus, while it is 
reasonable to expect excessive mitochondrial ROS production to impact the nuclear genome and whole-cell function, it may do so more often as a signaling molecule than through direct damage. Indeed, mitochondrial ETC function appears to affect such diverse processes as a cell-non-autonomous pathway of organismal longevity (Durieux et al. 2011) and nuclear gene expression (Droge 2002).

\section{Conclusions and outlook}

Our study provides a direct comparison of net ROS levels, 8-oxo-dG content and per-generation base substitution rate within long-term mutation-accumulation lines of C. elegans. Only two MA lines were observed to suffer significantly elevated relative ROS levels; though all MA lines consistently exhibited insignificant increases in both ROS and 8-oxo-dG content above the levels of the N2 progenitor line. Further, while we uncovered a strong, positive correlation between mitochondrial ROS and 8-oxo-dG levels, we found no such relationship between these two measures of oxidative stress and previously reported rates of heritable nuclear mutation. These findings are consistent with the notion that G-to-T transversions may not represent the most important signature of oxidative damage within the germline, though a larger study may have revealed weak associations.

Though the current study represents a step forward in our understanding of the relationship between oxidative stress and heritable mutation accumulation, several outstanding questions remain. Additional work will be necessary to determine whether large genome rearrangements more accurately reflect heritable oxidative damage than 
base substitution rates. Investigation into the potential causes of tissue-specificity in mutational processes could help to reveal the relative importance of oxidative damage and repair efficiency in preventing heritable mutation. Further work is also needed to describe the relationship between germline mtDNA mutations and steady-state ROS levels in C. elegans and other eukaryotes. Previous work indicates that C. briggsae, a sister species to $C$. elegans, experiences high rates of large mtDNA deletions (Howe et al. 2010) and decreased fitness compared to N2 (Baer et al. 2005). This, along with data showing significant variation in relative ROS levels among strains of $C$. briggsae (Estes et al., 2011; Hicks et al., 2012) suggest that large genomic perturbations of the mitochondrial genome may relate to oxidative stress in this species. Along with results such as these, the current study highlights the potential utility of $C$. elegans and related species as models for future work in this area. 


\section{CHAPTER FIVE}

Natural variation in Caenorhabditis briggsae mitochondrial form and function suggests a novel model of organelle dynamics

\section{Background}

Mitochondria are dynamic organelles that participate in continuous cycles of fusion, fission and autophagy within the cells of nearly all eukaryotic organisms. These cycles serve to link mitochondrial shape to organelle function (Chen and Chan 2005; Duvezin-Caubet et al. 2006) as well as each mitochondrion to the larger mitochondrial population (Hyde et al. 2010). Mitochondria perform several functions vital to eukaryotic life, including bioenergy (ATP) production and regulation of calcium homeostasis and apoptosis, nearly all of which depend upon the process of oxidative phosphorylation at the mitochondrial electron transport chain (ETC). Electron transfer through functional protein complexes of the ETC is coupled to the pumping of protons across the mitochondrial inner membrane, which establishes a mitochondrial membrane potential $(\Delta \Psi \mathrm{M})$. This $\triangle \Psi \mathrm{M}$ provides the potential energy to generate ATP and serves to control fusion-fission cycles (Twig et al. 2008a), both of which are necessary for mitosis, fuel sensing, autophagy and other processes (Mitra et al. 2009; Molina et al. 2009; Graef and Nunnari 2011). A natural consequence of ETC function is the occasional leakage of electrons onto molecular oxygen to generate reactive oxygen species (ROS) (Raha and Robinson 2000). Under normal circumstances, excess ROS are scavenged by various antioxidants before they can damage important macromolecules 
(Sedensky and Morgan 2006; Imlay 2008); however, impairment of the ETC often results in elevated ROS production (Grad and Lemire 2004; Verkaart et al. 2007; Dingley et al. 2009) and oxidative damage of proteins and nucleic acids (Wanagat et al. 2001; Yang et al. 2007), along with depressed $\triangle \Psi M$ (Ventura et al. 2006; Gaskova et al. 2007; Lemire et al. 2009) and altered mitochondrial dynamics (Ichishita et al. 2008).

Findings like those above highlight the integration between mitochondrial function, morphology and the fusion-fission cycle, and many recent studies have aimed to reveal the mechanistic bases of these relationships (Pham et al. 2004; Chen and Chan 2005; Palermo et al. 2007; Wikstrom et al. 2009; Westermann 2010; Yasuda et al. 2011). Mitochondrial ROS level does not appear to be related consistently to mitochondrial morphology or dynamics. For example, elevated mitochondrial ROS levels have been associated with both increased (Koopman et al. 2005) and decreased mitochondrial branching (Pletjushkina et al. 2006; Grünewald et al. 2010). Conversely, studies examining mitochondrial form and function in isolated cells and/or mutant organisms reveal a direct link between $\triangle \Psi \mathrm{M}$ and mitochondrial morphology, such that higher $\Delta \Psi \mathrm{M}$ induces organellar elongation (Legros et al. 2002; Ishihara et al. 2003) and loss of $\Delta \Psi M$ causes severe fragmentation of the mitochondrial network (Duvezin-Caubet et al. 2006; Song et al. 2007). Many of these mitochondrial shape changes are mediated by an altered balance between mitochondrial fusion and fission (Chen et al. 2005; Okamoto and Shaw 2005), which is increasingly appreciated to have a role in human disease. For instance, abnormal fusion-fission cycles are characteristic of neurodegenerative 
disorders including Parkinson's and Alzheimer's disease (Trimmer et al. 2000; BossyWetzel et al. 2003; Knott and Bossy-Wetzel 2008; Wang et al. 2008; Irrcher et al. 2010; Su et al. 2010; Winklhofer and Haass 2010). Many of the genes and cellular intermediates involved in mitochondrial dynamics (and its imbalance) have now been identified and characterized (Dimmer et al. 2002; Ishihara et al. 2003; Lee et al. 2004; Meeusen et al. 2004; Scorrano 2005; Griffin and Chan 2006). Based on such work, Twig and colleagues proposed that the fusion-fission-apoptosis cycle creates a "quality control axis" that acts to maintain mitochondrial integrity (Twig et al. 2008b). In their model, persistently depolarized mitochondria (those with low $\triangle \Psi M$ ) are segregated from the functional group by their inability to fuse. In this way low-functioning mitochondria - and perhaps, damaged mitochondrial genomes - are weeded out and an overall healthier organelle population is thus maintained (Twig et al. 2008b; Hyde et al. 2010; Kowald and Kirkwood 2011; Bess et al. 2012; Meyer and Bess 2012).

Despite the abundance of research focused on the dynamics of individual mitochondria (i.e., fusion and fission cycles), less attention has been devoted to the population-level behaviors of these organelles. A recent review highlights various "global" (cellular) and "local" (individual mitochondrion) controls thought to influence mitochondrial fusion and fission, suggesting that the collective mitochondrial population can indeed respond to cellular cues (Hyde et al. 2010). For example, mitochondria have been observed to undergo concerted hyper-fusion during G1-S phase of the cell cycle, and subsequent hyper-fragmentation as the cell progresses into $S$ phase (Hyde et al. 
2010). Still, we have a limited understanding of the biological roles of mitochondrial fission-fusion cycling and its organism-level consequences, and little information regarding the features and dynamics of mitochondrial populations and how these might influence individual mitochondrial form and function. Further, although we have some information about the patterns of relationship between certain mitochondrial phenotypes (e.g., $\triangle \Psi \mathrm{M}$ and organelle elongation), no comprehensive survey of such phenotypes has been conducted within live organisms. Finally, the extent to which research on cell lines and genetic fusion-fission mutants will apply to natural populations of organisms remains unknown.

Caenorhabditid nematodes have emerged as important models for studying the underlying causes of mitochondrial ETC dysfunction and its associated biological consequences. Mitochondrial metabolism and ETC function are known to be extremely similar in worms and mammals (reviewed in Dimmer et al., 2002; Westermann, 2010). Also, nematodes have highly differentiated tissues and a transparent cuticle that make them amenable to live imaging studies. Caenorhabditis briggsae in particular offers many advantages for mitochondrial biology research including its substantial mitochondrial genetic (Howe and Denver 2008) and phenotypic (Cutter et al. 2010; Raboin et al. 2010; Estes et al. 2011; Ross et al. 2011; Clark et al. 2012; Hicks et al. 2012) diversity. C. briggsae exhibit a cosmopolitan distribution and mitochondrial genetic analyses group its known natural isolates into three major phylogeographic clades corresponding to latitude of origin (Howe and Denver 2008) (Fig. 5.1). Recent work 
indicates that isolates within these clades are likely adapted to local thermal regimes (Jovelin and Cutter 2011; Prasad et al. 2011). We found that phylogenetic membership also accounts for among-isolate variation in several mitochondrial form and function traits; this was particularly true for $\Delta \Psi \mathrm{M}$, which was an extremely reliable predictor of

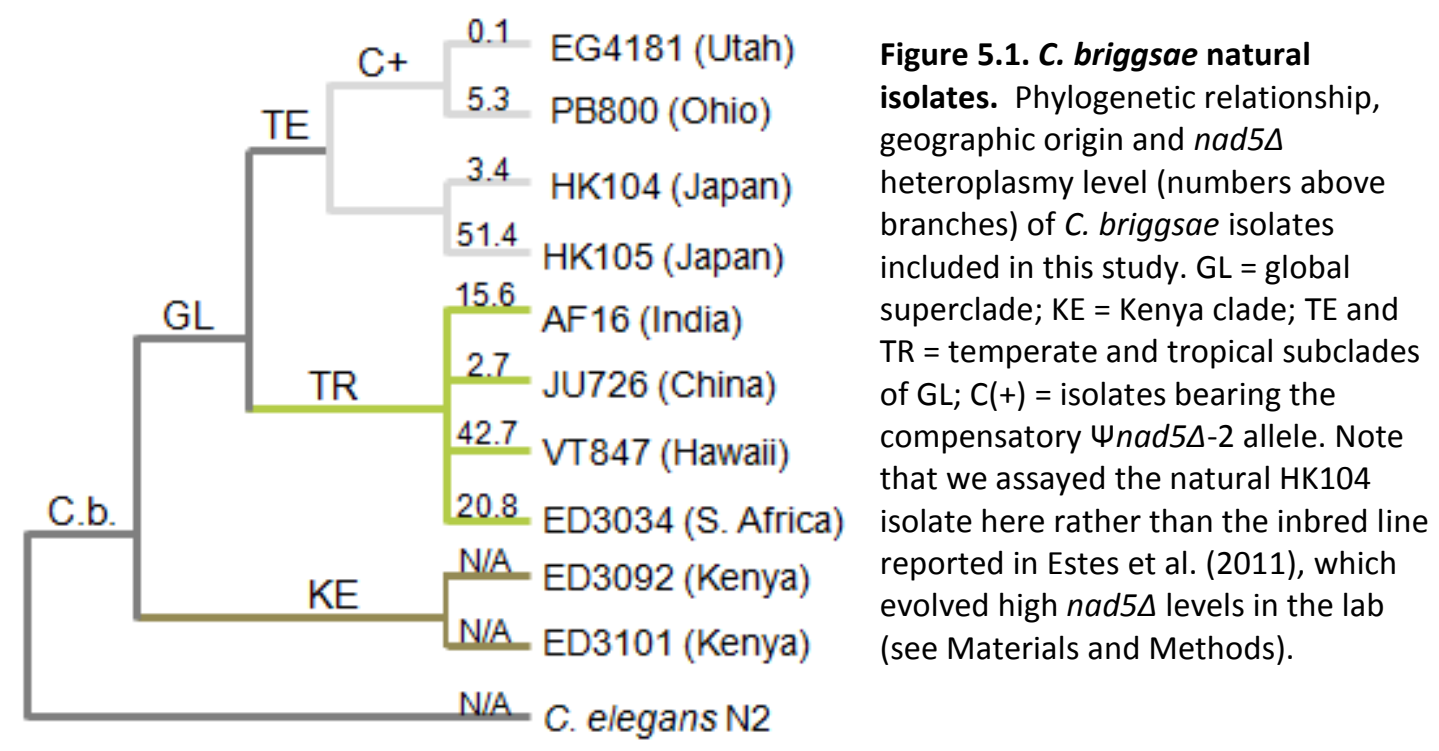

clade membership (see Chapter two, Table 2.2). Further, C. briggsae appear especially prone to acquiring mitochondrial deletion mutations (Howe et al. 2010), a process that has likely contributed to its high levels of standing mitochondrial genetic diversity. Indeed, many natural populations of $C$. briggsae harbor a large deletion (nad5 $\Delta$ ) within their mitochondrial genomes that removes half of the NADH-dehydrogenase 5 (nad5) gene (see Figure 1 in Howe \& Denver, 2008), which encodes an integral subunit of ETC complex I. nad5 $\Delta$-bearing genomes were recently shown to behave as selfish genetic elements (Clark et al. 2012) and levels of nad5 $\Delta$ heteroplasmy (the average number of deletion-bearing genomes per individual) are known to vary from zero to over $50 \%$ 
among geographically-segregated isolates of $C$. briggsae (Howe and Denver 2008; Estes et al. 2011). Recent work showed that nad5 level was unrelated to isolate-specific variation in $\triangle \Psi \mathrm{M}, \mathrm{ROS}$, and aspects of mitochondrial morphology (Hicks et al. 2012), but that it is likely to be detrimental to nematode health and fitness at high $(<\sim 40 \%)$ heteroplasmy levels (Howe and Denver 2008; Estes et al. 2011). In summary, its extensive genetic and subcellular phenotypic variation makes $C$. briggsae a promising natural system in which to investigate individual- and population-level mitochondrial behavior.

The present study is a reanalysis of data from our recent study of variation in $C$. briggsae mitochondrial form and function (Chapter two, Hicks et al. 2011), which quantified 24 mitochondrial phenotypes including ROS level, $\triangle \Psi M$ and aspects of organelle morphology in replicate live worms from 10 natural isolates of $C$. briggsae (Fig. 5.1) and reported the standing levels of phenotypic variation among clades and isolates. Here, the bivariate relationships of all mitochondrial phenotypes from the combined dataset were analyzed to examine the connections between mitochondrial physiology and dynamics within a natural system. Our findings support a major role for $\Delta \Psi M$ in shaping mitochondrial dynamics. Based on previous studies and current models of mitochondrial dynamics, we expected to observe more punctate morphologies among low- $\triangle \Psi \mathrm{M}$ mitochondria due to their reduced rates of fusion. Conversely, we expected that mitochondria with high $\triangle \Psi \mathrm{M}$ would maintain the canonical elongated shape. Our findings were in agreement with both of these expectations and provide general 
support for Twig's model (Twig et al. 2008b) of mitochondrial dynamics. Furthermore, our results suggest an addition to this model in which individual organelles respond to their functional environment; i.e., the average $\triangle \Psi M$ of the surrounding mitochondrial population.

\section{Materials and Methods}

\section{$\underline{\text { Nematode strains }}$}

We used data from Chapter two in which an array of mitochondrial phenotypes were measured for ten natural isolates of Caenorhabditis briggsae nematodes (Fig. 5.1). These isolates represent three major phylogeographic clades of $C$. briggsae and encompass the full range of known nad5 heteroplasmy level - from zero to $~ 50 \%$ deletion-bearing genomes. Briefly, the appearance of nad5 $\Delta$ depends upon the presence of a mitochondrial pseudogene - Unad5-2 (see Fig. 2.1 and Figure 1 from Howe \& Denver, 2008). The two Temperate clade isolates (PB800 and EG4181) harbor a compensatory $\Psi$ nad5-2 allele that limits the recurrent formation of nad5 $\Delta$; the two Kenyan clade isolates (ED3101 and ED3092) completely lack $\Psi$ nad5-2, which precludes formation of nad5 (Howe and Denver 2008). C. briggsae strains and the evolutionary genetics of nad5 5 have been described in further detail elsewhere (Howe and Denver 2008; Estes et al. 2011; Hicks et al. 2012).

\section{Sample preparation and image analysis}


For more detailed methods regarding nematode sample preparation, image acquisition and analysis, please refer to (Chapter two Material and Methods; Dingley et al., 2009; Estes et al., 2011; Hicks et al., 2012). Briefly, data for all mitochondrial traits were obtained by analyzing confocal images of the pharyngeal bulb region of young adult nematodes. Worms were incubated with $10 \mu \mathrm{m}$ concentrations of the mitochondria-targeted fluorescent dye(s) appropriate for each experiment (below). After 24 hours, worms were washed free of dye, paralyzed using levamisole, and imaged using a high-resolution wide-field confocal microscope (Advanced Light Microscopy Core, Oregon Health and Science University). All images were deconvolved prior to analysis and all image analysis was performed using ImageJ software (NIH).

The relative intensity of MitoSox Red (Molecular Probes, Eugene, OR) fluorescence from the terminal pharyngeal bulb of each worm was used to quantify relative ROS levels. Zielonka and Kalyanaraman (2010) determined that MitoSOX Red quantifies total levels of mitochondrial oxidants when used in conjunction with microscopic analysis (Zielonka and Kalyanaraman 2010). Final ROS levels for each isolate were calculated as the difference between pharyngeal bulb intensity in labeled and unlabeled control worms from each isolate. Dye-based ROS measurements reflect both the rates of ROS generation and ROS scavenging by antioxidant enzymes or small molecules, and thus give a comprehensive view of the level of oxidative stress experienced by an organism. Supporting this claim, a comparison of our ROS measurements with a survey of oxidative DNA damage (frequency of 8-oxo-dG lesions) 
conducted on a set of $C$. elegans mutation-accumulation lines (Denver et al. 2009, 2012) was highly positively correlated (Spearman's $\rho=0.943, P<=0.05$ ) (Fig. 4.2). Finally, we find no relationship between pharyngeal pumping rates and ROS or $\triangle \Psi \mathrm{M}$ (Estes et al. 2011; Hicks et al. 2012) indicating that our measures are not biased by variation in the rates of dye uptake by feeding.

Relative $\triangle \Psi M$ levels were quantified using MitoTracker Red CMXRos (Molecular Probes), a dye that localizes exclusively to polarized organelles (Pendergrass et al. 2004). The $\Delta \Psi M$ assays were performed concurrently with those of mitochondrial morphology by co-labeling worms with the $\Delta \Psi \mathrm{M}$-dependent probe MitoTracker Red CMXRos, and with MitoTracker Green FM (Molecular Probes), which accumulates within all mitochondria regardless of their respiration state (Pendergrass et al. 2004). This experimental setup allowed us to detect state-specific mitochondrial traits, such as shape changes occurring only in depolarized mitochondria, and to directly correlate mitochondrial $\triangle \Psi M$ and morphology traits. Unfortunately, the spectral similarities between the ROS and $\triangle \Psi M$ probes make it necessary to use separate images for ROS analysis. Thus, associations between ROS and all other mitochondrial traits should be interpreted with caution.

Finally, as previously discussed (Chapter two Materials and Methods), we failed to co-label nematodes treated as above with either DAPI or Hoechst 33342 (Sigma), which would have allowed us to visualize cell nuclei and thereby assess the intracellular distributions of mitochondria. (Appropriate GFP fusions are not yet available for $C$. 
briggsae.) Both dyes noticeably interfered with the fluorescence of the above MitoTracker dyes in C. briggsae (Hicks, pers. obs.). Our study therefore focuses on properties of individual mitochondria and mitochondrial populations within the pharyngeal bulb organ.

\section{$\underline{\text { Trait descriptions and statistical analysis }}$}

A total of 24 mitochondrial form and function traits were analyzed (Table 5.1). Briefly, relative mitochondrial membrane potential $(\triangle \Psi \mathrm{M}$ max) served as an indicator of mitochondrial functionality (see below). Relative reactive oxygen species (ROS max) further characterized mitochondrial activity. Maximum rather than mean $\triangle \Psi M$ and ROS

Label Trait Description

Measures of Mitochondrial Physiology

\begin{tabular}{|c|c|c|}
\hline $\begin{array}{l}\Delta \Psi \mathrm{M} \\
\operatorname{Max}\end{array}$ & Membrane potential & $\begin{array}{l}\text { Average of max relative MitoTracker Red CMXRos } \\
\text { fluorescence }\end{array}$ \\
\hline $\begin{array}{l}\text { ROS } \\
\text { Max }\end{array}$ & Reactive Oxygen Species & Average of max relative MitoSOX Red fluorescence \\
\hline \multicolumn{3}{|c|}{ Measures of the Mitochondrial Population } \\
\hline $\begin{array}{l}A_{F P} \\
A_{N P} \\
A_{T P}\end{array}$ & $\begin{array}{l}\text { Area of mitochondrial } \\
\text { population }\end{array}$ & $\begin{array}{l}\text { Area of functional, non-functional or total (both } \\
\text { functional and non-functional) mitochondrial } \\
\text { populations }\end{array}$ \\
\hline$A_{F P / N P}$ & $\begin{array}{l}\text { Ratio of functional to non- } \\
\text { functional mitochondrial } \\
\text { area }\end{array}$ & $\begin{array}{l}\text { Area of the functional mitochondrial population/area of } \\
\text { the non-functional population }\end{array}$ \\
\hline$A_{F P / T P}$ & $\%$ functional area & Area of the functional mitochondrial population/area of \\
\hline
\end{tabular}


the total population

\begin{tabular}{|c|c|c|}
\hline $\mathrm{N}_{\mathrm{F}}$ & \multirow{3}{*}{ Number of mitochondria } & \multirow{3}{*}{$\begin{array}{l}\text { Number of functional, non-functional, or total individual } \\
\text { mitochondria }\end{array}$} \\
\hline $\mathrm{N}_{\mathrm{N}}$ & & \\
\hline $\mathrm{N}_{\mathrm{T}}$ & & \\
\hline $\mathrm{N}_{\mathrm{F} / \mathrm{N}}$ & $\begin{array}{l}\text { Ratio of functional to non- } \\
\text { functional mitochondria }\end{array}$ & $\begin{array}{l}\text { Number of functional mitochondria/number of non- } \\
\text { functional mitochondria }\end{array}$ \\
\hline $\mathrm{N}_{\mathrm{F} / \mathrm{T}}$ & $\%$ functional mitochondria & $\begin{array}{l}\text { Number of functional mitochondria/number of total } \\
\text { mitochondria }\end{array}$ \\
\hline \multicolumn{3}{|c|}{ Measures of Individual Mitochondrial Shape } \\
\hline$A_{F}$ & \multirow{2}{*}{$\begin{array}{l}\text { Area of individual } \\
\text { mitochondria }\end{array}$} & \multirow{2}{*}{$\begin{array}{l}\text { Average area of individual functional or non-functional } \\
\text { mitochondria }\end{array}$} \\
\hline$A_{N}$ & & \\
\hline$A R_{F}$ & \multirow{2}{*}{ Aspect ratio } & \multirow{2}{*}{$\begin{array}{l}\text { Average of the ratio between the major and minor axis } \\
\text { of the ellipse equivalent to each functional or non- } \\
\text { functional mitochondrion }\end{array}$} \\
\hline$A R_{N}$ & & \\
\hline $\mathrm{AR}_{\mathrm{FV}}$ & \multirow{2}{*}{ Aspect ratio variance } & \multirow{2}{*}{$\begin{array}{l}\text { Average within-individual variance in aspect ratio of } \\
\text { functional or non-functional mitochondria }\end{array}$} \\
\hline $\mathrm{AR}_{\mathrm{NV}}$ & & \\
\hline $\mathrm{C}_{\mathrm{F}}$ & \multirow{2}{*}{ Circularity } & \multirow{2}{*}{$\begin{array}{l}4 \prod\left(\text { area/perimeter }{ }^{2} \text { ) for functional or non-functional }\right. \\
\text { mitochondria }\end{array}$} \\
\hline $\mathrm{C}_{\mathrm{N}}$ & & \\
\hline $\mathrm{C}_{\mathrm{FV}}$ & \multirow{2}{*}{ Circularity variance } & \multirow{2}{*}{$\begin{array}{l}\text { Within-individual variance in circularity of functional or } \\
\text { non-functional mitochondria }\end{array}$} \\
\hline $\mathrm{C}_{\mathrm{NV}}$ & & \\
\hline
\end{tabular}

Table 5.1. Description of mitochondrial traits measured in C. briggsae.

levels were used because we previously found a significant effect of levamisole (the cholinergic agonist used to paralyze nematodes for image acquisition) on mean but not maximum ROS levels (Chapter two Materials and Methods). Additionally, we scored ten traits that describe aspects of the pharyngeal mitochondrial population: the combined 
area of the mitochondrial population $\left(A_{F P}, A_{N P}, A_{T P}\right)$, the ratio of the area of functional to nonfunctional mitochondria $\left(\mathrm{A}_{\mathrm{FP} / \mathrm{NP}}\right)$, and the percentage of the total mitochondrial area that is functional $\left(A_{F P / T P}\right)$, the number of organelles $\left(N_{F}, N_{N}, N_{T}\right)$, the ratio of functional to nonfunctional organelles $\left(\mathrm{N}_{\mathrm{F} / \mathrm{N}}\right)$, and the percentage of functional mitochondria $\left(\mathrm{N}_{\mathrm{F} / \mathrm{T}}\right)$. Functional mitochondria were distinguished by their quantifiable uptake of MitoTracker Red CMXRos. Differences in mitochondrial morphology were measured using the area $\left(A_{F}, A_{N}\right)$, aspect ratio $\left(A R_{F}, A R_{N}\right)$, and circularity $\left(C_{F}, C_{N}\right)$ of individual mitochondria. Aspect ratio quantifies elongation and has a minimal value of 1 , which corresponds to a perfect circle (Russ 2002). Circularity will also equal 1 when the measured object is a perfect circle, but decreases to 0 as the object becomes more branched (Russ 2002). Finally, the variance in circularity $\left(C_{\mathrm{FV}}, \mathrm{C}_{\mathrm{NV}}\right)$ and aspect ratio $\left(A R_{\mathrm{FV}}, A R_{\mathrm{NV}}\right)$ measured the degree of heterogeneity within the mitochondrial population of each nematode.

Because our data often violated assumptions of the Pearson product-moment correlation (e.g., normally distributed data, monotonic bivariate relationships), we characterized correlations among mitochondrial form and function characters by calculating Spearman rank-order correlation coefficients between each pair of traits as in Huang et al. (2004). Because ROS levels were measured on different sets of nematodes than all other traits, we measured isolate-mean correlations for these pairs of traits. All statistical analysis was performed in JMP 9 (SAS Institute, Cary, NC).

\section{Results}




\section{Mitochondrial trait associations}

We analyzed the relationships between pairs of mitochondrial traits (Table 5.1) originally obtained in Chapter two for all natural isolates following (Estes et al. 2011). First, no significant correlations between ROS and any other mitochondrial trait were revealed (data not shown). Maximum $\triangle \Psi M$ was, however, statistically related to a number of other characters. $\triangle \Psi \mathrm{M}$ was positively related to aspect ratio of functional mitochondria $\left(A R_{F}\right)(\rho=0.208, P<=0.01)$, meaning that isolates with higher maximum $\Delta \Psi \mathrm{M}$ tended to have more elongated mitochondria. Similarly, maximum $\Delta \Psi \mathrm{M}$ was also weakly negatively correlated to the circularity of functional mitochondria $\left(C_{F}\right)(\rho=-0.248$, $P<=0.01)$, suggesting that worms with higher maximum $\Delta \Psi \mathrm{M}$ fluorescence also tended to have less circular - or more branched - organelles. It is important to note that maximum $\triangle \Psi \mathrm{M}$ was necessarily positively correlated to traits related to functional mitochondrial area $\left(A_{F}, A_{F P}\right.$, and $\left.A_{T P}\right)$ since individuals with higher scores for these traits had necessarily taken up more membrane-potential dependent dye and thus had higher values for $\triangle \Psi \mathrm{M}$.

A number of the other correlations were expected due to the nature of the measurements (e.g., between traits describing the number of mitochondria and those describing the combined area of mitochondria populations); however, a systematic survey of the remaining (statistically significant) correlations revealed consistent patterns of relationship between the major classes of mitochondrial traits (shape, area, and number), which can be summarized as follows: 
(1) As expected, circularity $\left(C_{N}\right.$ and $\left.C_{F}\right)$ demonstrated a strong negative correlation with aspect ratio $\left(A R_{N}\right.$ and $\left.A R_{F}\right)$ for both nonfunctional and functional mitochondria ( $\rho=-0.811, P<=0.001$ and $\rho=-0.801, P<=0.001$, respectively). Figure $5.2 \mathrm{~A}$ shows the relationship between these two traits for functional mitochondria. Circularity responds to changes in surface irregularities (or the amount of branching) of each mitochondrion whereas aspect ratio responds to the elongation of organelles (Russ 2002; Koopman et al. 2005). This negative relationship must certainly owe itself largely to the fact that more circular mitochondria are less elongate; however, it also implies that mitochondrial branching was rare and that deviations from perfect circularity were most often achieved by elongation rather than by branching for all organelles regardless of their functional status.

(2) In isolates containing a higher ratio of polarized mitochondria (higher scores for $\mathrm{N}_{\mathrm{F} / \mathrm{N}}$ or $\mathrm{N}_{\mathrm{F} / \mathrm{T}}$ ), all mitochondria were larger and less circular (e.g., Fig. 5.2B shows this pattern for functional mitochondria) and more elongate regardless of their functional state. However, only the depolarized mitochondria in these isolates were significantly more variable with regard to circularity (higher scores for $C_{N V}$ ). Similarly, as the area of individual functional mitochondria $\left(A_{F}\right)$ or the combined area of the functional 

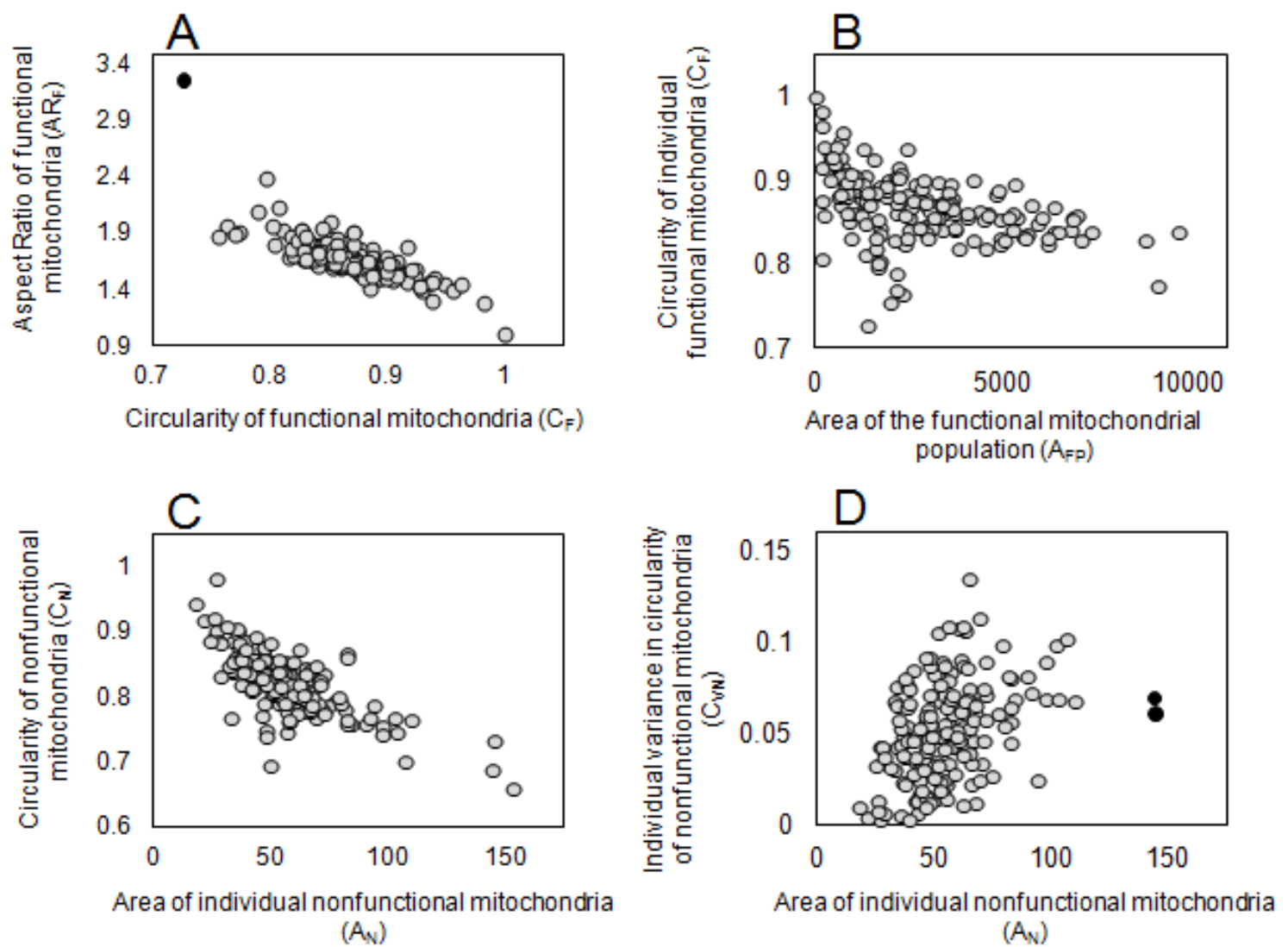

Figure 5.2. Examples of bivariate relationships of mitochondrial phenotypes. Patterns of relationship between traits describing mitochondrial size, morphology, and within-individual variance are shown. All measurements were made on the same set of confocal images (see Materials and Methods) and each point represents the bivariate phenotype for an individual nematode ( $N=167-170)$. A) Aspect ratio is negatively related to circularity within the functional mitochondria of individual worms $(\rho=-0.806$, $P<=0.0001)$. Removing one outlier (black symbol) decreases the correlation slightly $(\rho=-0.797$, $P<=0.0001)$. B) As the two-dimensional area of the total functional mitochondrial population increases, functional mitochondria become less circular $(\rho=-0.509, P<=0.0001)$. C) As the two-dimensional area of individual nonfunctional mitochondria increases, these mitochondria become less circular $(\rho=-0.698$, $P<=0.0001)$. D) As the two-dimensional area of the total functional mitochondrial population increases, the nonfunctional organelles within each worm become variable with respect to circularity. When two outliers (black symbols) are removed, the correlation remains unchanged $(\rho=0.409, P<=0.0001)$.

mitochondrial population (higher scores for $A_{F P}$ ) increased, all mitochondria became less circular and more elongate.

(3) In isolates with more nonfunctional/depolarized mitochondria (higher scores for $N_{N}$ ), the nonfunctional mitochondria in these isolates become less elongate and 
more uniform with respect this trait (lower scores for $A R_{N}$ and $A R_{N V}$, respectively). Conversely, the polarized mitochondria in these isolates were more variable with regard to elongation (higher scores for $A R_{F V}$ ). As the area of individual nonfunctional mitochondria $\left(A_{N}\right)$ or the area of the nonfunctional mitochondrial population $\left(A_{N P}\right)$ increased, nonfunctional mitochondria responded by deviating from circularity (lower scores for $C_{N}$, indicative of elongation or branching, Fig. 5.2C) (c.f., Koopman et al., 2005) and by becoming more variable with respect to circularity (higher scores for $\mathrm{C}_{\mathrm{NV}}$, Fig. 5.2D). Because we omitted mitochondria smaller than 2 pixels from all analyses (Hicks et al., 2012), the strength of these correlations and the fact that they apply only to depolarized mitochondria suggest that they should not be influenced by any size-related bias.

Finally, we note that regressions of average mitochondrial trait correlations for each isolate on isolate-specific nad5 $\Delta$ heteroplasmy revealed no evidence that any of the mitochondrial phenotypic associations were related to nad5 $\Delta$ level; however, because we had estimates only of average nad5 for each C. briggsae isolate obtained from a different set of worms than those phenotyped (Hicks et al. 2012), the biological meaning of these tests is questionable.

\section{Discussion}

$\underline{\text { Implications for mitochondrial dynamics }}$ 
We performed a systematic evaluation of phenotypic correlations among mitochondrial traits originally reported in (Hicks et al., 2012) with the aim of uncovering patterns that describe the relationships between mitochondrial physiology and morphology. While some studies have indicated that ROS production can be associated with dramatic ultra-structural transformations in mitochondria (Koopman et al. 2005; Wang et al. 2008; Liot et al. 2009), our analyses failed to clearly relate net ROS levels with any alterations in mitochondrial shape or population structure. Again, a caveat prohibiting further interpretation of this result is that ROS was necessarily measured on different individual nematodes than all other mitochondrial traits (see Materials and Methods). Thus, estimates of ROS were obtained from different sets of mitochondria than those describing $\triangle \Psi M$ and morphology traits.

In agreement with previous studies (Ishihara et al. 2003; Mattenberger et al. 2003; Twig et al. 2008a), our findings suggest a central role for $\Delta \Psi M$ in shaping mitochondrial morphology, manifested in its relationship with several aspects of mitochondrial shape and population structure (Table 5.2). In particular, our analysis of mitochondrial trait associations revealed that mitochondria appear to respond differently depending on their functional neighborhood; i.e., whether they are surrounded by other organelles that are mainly polarized or depolarized. The morphology and physiological state of individual mitochondria is dependent on $\triangle \Psi M$ (Ishihara et al. 2003; Miceli et al. 2011; Twig and Shirihai 2011). This makes sense as several critical organellar functions are contingent upon $\triangle \Psi \mathrm{M}$, including mitochondrial 


\begin{tabular}{|c|c|c|c|c|c|c|c|}
\hline & $N_{T}$ & $N_{N}$ & $N_{F / T}$ & $N_{F / N}$ & $N_{F}$ & $\Delta \Psi M_{\max }$ & $c_{\mathrm{NV}}$ \\
\hline$A_{F}$ & $0.220 * *$ & 0.018 & $0.317 * * *$ & $0.3217 * * *$ & $0.306 * * *$ & 0.121 & -0.042 \\
\hline$A_{F P}$ & $0.739 * * *$ & $0.210 * *$ & $0.684 * * *$ & $0.684 * * *$ & $0.892 * * *$ & $0.422 * * *$ & 0.045 \\
\hline$A_{F P / N P}$ & $0.297 * * *$ & $-0.277 * * *$ & $0.828 * * *$ & $0.828 * * *$ & $0.638 * * *$ & $0.312 * * *$ & -0.006 \\
\hline$A_{\mathrm{FP} / \mathrm{TP}}$ & $0.297 * * *$ & $-0.277^{* * *}$ & $0.828 * * *$ & $0.828 * * *$ & $0.638 * * *$ & $0.312 * * *$ & -0.006 \\
\hline$A_{N}$ & 0.027 & -0.086 & $0.168^{*}$ & $0.168^{*}$ & 0.112 & -0.124 & $0.409 * * *$ \\
\hline$A_{N P}$ & $0.649 * * *$ & $0.821 * * *$ & $-0.269 * * *$ & $-0.269 * * *$ & $0.294 * * *$ & 0.083 & 0.018 \\
\hline$A R_{F}$ & 0.039 & -0.091 & $0.183^{* *}$ & $0.183^{*}$ & 0.12 & $0.208^{*}$ & 0.051 \\
\hline $\mathrm{AR}_{\mathrm{FV}}$ & 0.069 & $0.154^{*}$ & -0.107 & -0.107 & 0.004 & 0.080 & -0.043 \\
\hline$A R_{N}$ & -0.055 & $-0.200 * *$ & $0.188^{*}$ & $0.188^{*}$ & 0.109 & -0.110 & $0.478 * * *$ \\
\hline$A R_{\mathrm{NV}}$ & -0.135 & $-0.241 * *$ & 0.151 & 0.151 & -0.002 & -0.033 & $0.677^{* * *}$ \\
\hline$A_{\text {TP }}$ & $0.898 * * *$ & $0.604 * * *$ & $0.310 * * *$ & $0.310 * * *$ & $0.759 * * *$ & $0.334 * * *$ & 0.049 \\
\hline$C_{F}$ & $-0.211^{* *}$ & -0.007 & $-0.276 * * *$ & $-0.276 * * *$ & $-0.273 * * *$ & $-0.248 * * *$ & -0.031 \\
\hline $\mathrm{C}_{\mathrm{Fv}}$ & 0.107 & 0.120 & 0.012 & 0.012 & 0.105 & 0.113 & -0.036 \\
\hline$c_{N}$ & -0.096 & 0.062 & $-0.196 * *$ & $-0.196 * *$ & $-0.189 *$ & 0.051 & $-0.522 * * *$ \\
\hline $\mathrm{C}_{\mathrm{NV}}$ & -0.078 & $-0.223 * *$ & $0.190 *$ & $0.190 *$ & 0.088 & 0.024 & \\
\hline$\Delta \Psi \mathrm{M} \max$ & $0.414 * * *$ & 0.147 & $0.311 * * *$ & $0.311 * * *$ & $0.465 * * *$ & & \\
\hline $\mathbf{N}_{\mathrm{F}}$ & $0.805^{* * *}$ & $0.273 * * *$ & $0.6884 * * *$ & $0.688 * * *$ & & & \\
\hline$N_{F / N}$ & $0.268 * * *$ & $-0.413 * * *$ & $1 * * *$ & & & & \\
\hline$N_{F / T}$ & $0.265 * * *$ & $-0.413 * * *$ & & & & & \\
\hline $\mathbf{N}_{\mathrm{N}}$ & $0.724 * * *$ & & & & & & \\
\hline $\mathbf{N}_{\mathbf{T}}$ & & & & & & & \\
\hline
\end{tabular}




\begin{tabular}{|c|c|c|c|c|c|c|c|}
\hline & $c_{N}$ & $\mathrm{C}_{\mathrm{FV}}$ & $c_{F}$ & $A_{\text {TP }}$ & $\mathrm{AR}_{\mathrm{NV}}$ & $A R_{N}$ & $\mathbf{A R}_{\mathrm{FV}}$ \\
\hline$A_{F}$ & $-0.171^{*}$ & $0.369 * * *$ & $-0.709 * * *$ & $0.447 * * *$ & -0.051 & $0.188^{*}$ & $0.195^{*}$ \\
\hline$A_{F P}$ & $-0.216 * *$ & $0.206^{*}$ & $-0.509 * * *$ & $0.813 * * *$ & -0.017 & $0.159 *$ & 0.055 \\
\hline$A_{\mathrm{FP} / \mathrm{NP}}$ & 0.019 & 0.145 & $-0.531 * * *$ & $0.319 * * *$ & -0.005 & 0.058 & -0.004 \\
\hline$A_{F P / T P}$ & 0.019 & 0.145 & $-0.531 * * *$ & $0.329 * * *$ & -0.005 & 0.058 & -0.004 \\
\hline$A_{N}$ & $-0.698 * * *$ & 0.035 & 0.05 & $0.323 * * *$ & $0.315^{* * *}$ & $0.510 * * *$ & -0.008 \\
\hline$A_{\mathrm{NP}}$ & $-0.316 * * *$ & 0.106 & 0.048 & $0.716^{* * *}$ & -0.038 & 0.092 & 0.119 \\
\hline $\mathrm{AR}_{\mathrm{F}}$ & -0.064 & $0.352 * * *$ & $-0.801 * * *$ & 0.097 & -0.027 & 0.085 & $0.342 * * *$ \\
\hline$A R_{\mathrm{FV}}$ & 0.006 & $0.698 * * *$ & $-0.313 * * *$ & 0.100 & -0.075 & -0.020 & \\
\hline$A R_{N}$ & $-0.811 * * *$ & -0.009 & -0.103 & 0.149 & $0.544 * * *$ & & \\
\hline $\mathrm{AR}_{\mathrm{NV}}$ & $-0.420 * * *$ & -0.048 & 0.059 & -0.031 & & & \\
\hline$A_{T P}$ & $-0.334 * * *$ & $0.179 *$ & $-0.325 * * *$ & & & & \\
\hline$C_{F}$ & 0.131 & $-0.452 * * *$ & & & & & \\
\hline $\mathrm{C}_{\mathrm{FV}}$ & -0.045 & & & & & & \\
\hline \multicolumn{8}{|l|}{$C_{N}$} \\
\hline \multicolumn{8}{|l|}{$\mathrm{c}_{\mathrm{NV}}$} \\
\hline \multicolumn{8}{|c|}{$\Delta \Psi M \max$} \\
\hline \multicolumn{8}{|l|}{$\mathbf{N}_{\mathbf{F}}$} \\
\hline \multicolumn{8}{|l|}{$N_{F / N}$} \\
\hline \multicolumn{8}{|l|}{$\mathbf{N}_{\mathrm{F} / \mathrm{T}}$} \\
\hline \multicolumn{8}{|l|}{$\mathbf{N}_{\mathrm{N}}$} \\
\hline $\mathbf{N}_{\mathrm{T}}$ & & & & & & & \\
\hline
\end{tabular}




\begin{tabular}{|l|l|l|l|l|l|l|} 
& $\mathrm{AR}_{\mathrm{F}}$ & $\mathbf{A}_{\mathrm{NP}}$ & $\mathbf{A}_{\mathrm{N}}$ & $\mathbf{A}_{\mathrm{FP} / \mathrm{TP}}$ & $\mathbf{A}_{\mathrm{FP} / \mathrm{NP}}$ & $\mathbf{A}_{\mathrm{FP}}$ \\
\hline $\mathbf{A}_{\mathrm{F}}$ & $0.428^{* * *}$ & 0.028 & 0.066 & $0.632^{* * *}$ & $0.632^{* * *}$ & $0.645^{* * *}$ \\
\hline $\mathbf{A}_{\mathrm{FP}}$ & $0.256^{* * *}$ & $0.228^{* *}$ & 0.107 & $0.782^{* * *}$ & $0.782^{* * *}$ & \\
\hline $\mathbf{A}_{\mathrm{FP} / \mathrm{NP}}$ & $0.352^{* * *}$ & $-0.362^{* * *}$ & $-0.181^{*}$ & $1 * * *$ & & \\
\hline $\mathbf{A}_{\mathrm{FP} / \mathrm{TP}}$ & $0.352^{* * *}$ & $-0.362^{* * *}$ & $-0.181^{*}$ & & & \\
\hline $\mathbf{A}_{\mathrm{N}}$ & -0.067 & $0.449 * *$ & & & & \\
\hline $\mathbf{A}_{\mathrm{NP}}$ & -0.131 & & & & & \\
\hline $\mathbf{A R}_{\mathrm{F}}$ & & & & & & \\
\hline $\mathbf{A R}_{\mathrm{FV}}$ & & & & & & \\
\hline $\mathbf{A R}_{\mathrm{N}}$ & & & & & & \\
\hline $\mathbf{A R}_{\mathrm{NV}}$ & & & & & & \\
\hline $\mathbf{A}_{\mathrm{TP}}$ & & & & & & \\
\hline $\mathbf{C}_{\mathrm{F}}$ & & & & & & \\
\hline $\mathbf{C}_{\mathrm{FV}}$ & & & & & & \\
\hline $\mathbf{C}_{\mathrm{N}}$ & & & & & & \\
\hline $\mathbf{C}_{\mathrm{NV}}$ & & & & & & \\
\hline$\Delta \boldsymbol{\Psi} \mathbf{M} \mathbf{m a x}$ & & & & & & \\
\hline $\mathbf{N}_{\mathrm{F}}$ & & & & & & \\
\hline $\mathbf{N}_{\mathrm{F} / \mathrm{N}}$ & & & & & & \\
\hline $\mathbf{N}_{\mathrm{F} / \mathrm{T}}$ & & & & & & \\
\hline $\mathbf{N}_{\mathrm{N}}$ & & & & & & \\
\hline $\mathbf{N}_{\mathrm{T}}$ & & & & & & \\
\hline
\end{tabular}

Table 5.2. Among-trait correlations in C. briggsae natural isolates

fusion and ATP and ROS production rates (Ishihara et al. 2003; Gaskova et al. 2007; Murphy 2009). Recent work has shown that mitochondrial fusion is brief and accompanied by fission (Twig et al. 2008a; Wikstrom et al. 2009) and that normal cycles of fusion and fission are necessary to maintain the canonical ovoid mitochondrial shape (Chen and Chan 2005; Kageyama et al. 2011). The model of mitochondrial life cycles 
proposed by Twig, et al. (2008b) connects mitochondrial morphology and function by suggesting that, following a fusion-fission cycle, one daughter mitochondrion remains polarized while the other is transiently depolarized. The transiently depolarized daughter will either regain $\Delta \Psi M$ (if it contains a sufficient number of functional ETC components) and resume its participation in the fusion-fission cycle, or it will remain depolarized and undergo fission and eventual mitophagic degradation (See Figure 1 in Twig, et al., 2008b). Our data suggest that such fusion-fission cycling occurs within the context of a larger mitochondrial population that is itself either more or less polarized (Fig. 5.3). Specifically, we find that both polarized and depolarized mitochondria are more elongate (less fragmented) in C. briggsae isolates containing more mitochondria with high $\triangle \Psi M$ (higher values for $N_{F / N}$ and $A_{F P}$ ), although depolarized organelles are slightly more variable in shape than polarized organelles. Conversely, when they inhabit less functional isolates (those with more mitochondria with low $\Delta \Psi \mathrm{M}$ ), both polarized and depolarized mitochondria become increasingly heterogeneous in shape, but depolarized mitochondria become overall more fragmented (Table 5.2). Placing these data within the context of Twig et al.'s (2008b) model, we propose that a majority of the mitochondria in isolates with higher average $\Delta \Psi \mathrm{M}$ will exhibit the "typical" ovoid shape by maintaining normal rates of fusion-fission cycles. Transiently depolarized mitochondria in this environment will be more likely to recover membrane polarization after fission, helping to maintain a large polarized mitochondrial population. Conversely, isolates with lower $\Delta \Psi \mathrm{M}$ will suffer a reduced frequency of fusion-fission cycling and 
display increased shape heterogeneity in the entire mitochondrial population. Transiently depolarized mitochondria in this environment will be less likely to harbor functional ETC products and will more often join the persistently depolarized population, which is unable to undergo fusion. Polarized mitochondria in these isolates will then experience reduced numbers of fusion "mates" - in essence, an intracellular Allee effect (Allee 1931), which will contribute to further reduced rates of fusion-fission cycling and lead to increased shape heterogeneity of all mitochondrial morphs (Fig. $5.3)$.

\section{MITOCHONDRIAL ENVIRONMENT}

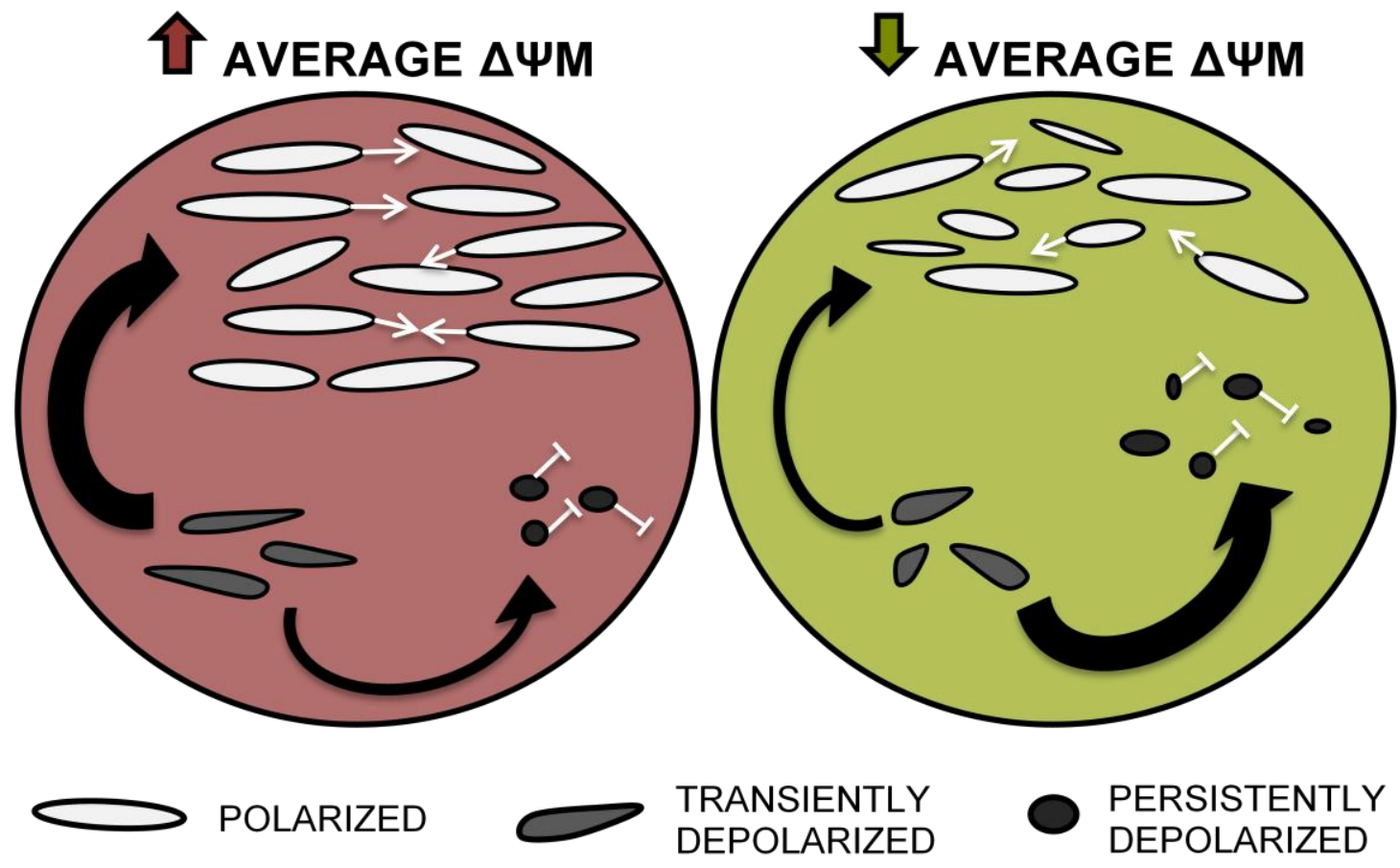


Figure 5.3. A context-dependent model of mitochondrial dynamics in which mitochondria respond to the functional state of their intracellular environment. An organism has three types of mitochondrial populations, polarized, transiently depolarized, and persistently depolarized. The polarized population is capable of undergoing fusion (white arrows) while the persistently depolarized population is not (white blunted arrow). The transiently depolarized mitochondria are produced after a fusion-fission cycle and will either regain sufficient $\Delta \psi \mathrm{M}$ and join the polarized/fusing population (gray arrow) or, if they are unable to regain $\Delta \psi \mathrm{M}$, join the persistently depolarized population (black arrow) (Twig et al., 2008b). We propose that mitochondria in a more functional environment (higher $\Delta \psi \mathrm{M}$, at left) are more likely to regain $\Delta \psi \mathrm{M}$ and join the polarized/fusing population. Normal fusion-fission cycles will maintain a majority of mitochondria in the canonical ovoid morph. Here, many depolarized mitochondria are destined to recover $\triangle \psi \mathrm{M}$ and rejoin the fusing population. Conversely, mitochondria in a less functional environment (lower $\Delta \psi \mathrm{M}$, at right) are less likely to regain $\Delta \psi \mathrm{M}$ and will therefore join the persistently depolarized/non-fusing population resulting in fewer polarized mitochondria. Here, lower than normal rates of fusion and fission will increase the shape heterogeneity of all mitochondria.

$\underline{\text { Implications for mitochondrial genome integrity and evolution }}$

It has been suggested that damaged mitochondrial genomes may be preferentially shunted to the depolarized daughter organelle - the one more often destined for degradation (Twig et al. 2008b; Kowald and Kirkwood 2011). If this is the case, mitochondrial fusion-fission cycling may have a critical role to play in maintaining mtDNA genome stability and could conceivably contribute to intra- and interspecific differences in mtDNA mutation rates and heteroplasmy levels. Recent work shows that the removal of damaged mtDNA in C. elegans requires mitochondrial fusion (Bess et al. 2012; Meyer and Bess 2012). Because the fusion-fission cycle relies on $\Delta \Psi \mathrm{M}$, alterations to $\triangle \Psi \mathrm{M}$ that are unrelated to mtDNA quality (i.e., that weaken the link between mitochondrial genotype and phenotype) could reduce the efficacy of the selective process (Twig et al. 2008b). In Chapter two, we determined that much of the measured variation in mitochondrial phenotypes - and especially that of $\Delta \Psi \mathrm{M}$ - related to the phylogeographic clade membership of particular $C$. briggsae isolates, rather than to 
nad5 level (Tables 2.2 and 2.3). Specifically, Tropical isolates tended to have the lowest values for $\triangle \Psi \mathrm{M}$ followed by Kenyan isolates, and Temperate isolates exhibited the highest $\Delta \Psi \mathrm{M}$ (Figure 2.3G). This led to the hypothesis that Tropical C. briggsae isolates may have adaptively reduced their $\Delta \Psi \mathrm{M}$ in order to counter increased ROS levels brought on by high temperatures (Brand 2000; Hicks et al. 2012). Because C. briggsae are ectotherms, the external temperature can directly influence their physiology; higher temperatures can increase nematode metabolism and ROS generation. Additionally, Tropical clade worms contain fewer total mitochondria $\left(\mathrm{N}_{\mathrm{T}}\right.$ and $\left.\mathrm{N}_{\mathrm{F}}\right)$ within the focal area (pharyngeal bulb) compared to both the Temperate and Kenyan clades (Tukey's HSD, $\alpha$ $=0.05 ;($ Figure $2.3 \mathrm{H}))$. Since cold-adapted ectotherms often exhibit increased mitochondrial density (Morley et al. 2009), the reduction in mitochondrial number within Tropical clade isolates is also consistent with an adaptive response to heat. If it is indeed the case that Tropical $C$. briggsae isolates have adaptively reduced their $\Delta \Psi \mathrm{M}$, they may experience a reduced efficiency of selection allowing the amplification of damaged mtDNA molecules - such as those bearing nad5L. Such a dynamic could help to explain the counter-intuitive finding that Tropical C. briggsae isolates display higher average nad5 $\Delta$ heteroplasmy levels despite having larger effective population sizes (and presumably more efficient natural selection) than Temperate clade isolates that have fixed compensatory mutations preventing nad5 accumulation (Howe and Denver 2008). In other words, the Tropical isolate's adaptive reduction of $\Delta \Psi M$ in response to high temperature may interfere with the selective removal of nad5 $\Delta$ by the 
mitochondrial fusion-fission cycle. Alternatively, because nad5 $\Delta$ affects a component of mitochondrial ETC complex I that is putatively involved in $\mathrm{H}^{+}$pumping (Janssen et al. 2006; Lenaz et al. 2006), the deletion may itself reduce $\Delta \Psi M$ and provide a ROS avoidance mechanism that does not directly produce heat (unlike mitochondrial uncoupling) - thus conferring a direct benefit to Tropical C. briggsae isolates (Brand 2000; Iser et al. 2005). Either scenario implies that different evolutionary pressures may be shaping the subcellular phenotypes of different $C$. briggsae populations and suggests fruitful avenues for experimental work.

\section{Conclusions}

Our analysis suggests that $\triangle \Psi \mathrm{M}$, but not mitochondrial ROS level, has a major role in shaping mitochondrial dynamics within natural populations of $C$. briggsae nematodes. We also identified a set of correlations that may describe a global control mechanism for mitochondrial dynamics. In particular, our findings suggest a model of mitochondrial population dynamics in which cellular environmental context dependency - and in particular, whether the mitochondrial population is mainly polarized or depolarized - is a key feature. To our knowledge, ours is the first study to connect natural variation in subcellular phenotypes to a model of mitochondrial dynamics. The model is also congruent with recent work highlighting the importance of both organellar and cellular influence on mitochondrial fusion-fission processes (Hyde et al. 2010; Kowald and Kirkwood 2011), but robust tests will require experimental confirmation of several assumptions, including whether $\triangle \Psi M$ correlates linearly to fission-fusion ability. 
The natural phenotypic and genetic variation within the $C$. briggsae system will be advantageous for further study in this area (e.g., partitioning of the genetic and cellular environmental components of observed mitochondrial phenotypic variation). With particular regard to $C$. briggsae evolution, our findings highlight the need for future work to understand what if any role mitochondrial fission-fusion dynamics play in mediating transmission of nad5 $\Delta$-bearing genomes and adaptation to local thermal and other conditions. 


\section{CHAPTER SIX}

Broad Conclusions

\section{Evolutionary Implications}

Together, these studies provide important, novel advancements in our understanding of the potential cell biological and evolutionary causes and consequences of mitochondrial genotypic and phenotypic variation. While a great deal of evolutionary study has focused on variation in organismal phenotypes such as fitness, we have very little knowledge of the amount of natural variation that exists in sub-cellular phenotypes. Thus, we lack a basic understanding of the importance of sub-cellular variation in organismal evolution. In the current study, we uncovered significant amongisolate variation in 18 of the 24 mitochondrial form and function traits assayed, showing that natural variation in important sub-cellular phenotypes does exist. Because mtDNA is known to experience purifying selection (Mishmar et al. 2003, 2006; Meiklejohn et al. 2007), the variation observed here has the potential to be adaptive. $\Delta \Psi \mathrm{M}$ displayed the greatest among-isolate variation within C. briggsae natural isolates, much of which was related to phylogeographic clade membership (Fig 2.3G, Table 2.2). It is possible that the depressed $\triangle \Psi \mathrm{M}$ observed within Tropical clade isolates is an adaptive response to reduce ROS production from the mitochondria. However, confirmation of lowered $\triangle \Psi \mathrm{M}$ and ROS at higher temperatures within Tropical isolates will be necessary to determine if this is the case. An assay examining the effect of mitochondrial uncouplers on ROS 
production rates and fitness at high temperatures will also be telling. Further, $\triangle \Psi M$ is important for several processes besides ROS production, including the transport of fatty acid components and ATP/ADP molecules (Vander Heiden et al. 2000), both of which are likely important for thermal adaptation. Thus, while the existence of substantial variation in mitochondrial $\triangle \Psi M$ is suggestive of adaptive evolution, further work will be necessary to identify the primary drivers of the adaptive evolution (reducing ROS production as opposed to altering ATP/ADP transport, for example), if it indeed has occurred.

Based on previous work in C. briggsae showing that high nad5 $\Delta$ deletion levels are related to decreased fitness (Howe and Denver 2008; Estes et al. 2011), we expected to see a correlation between ROS and $\triangle \Psi \mathrm{M}$ and isolate-specific nad5 5 heteroplasmy level. While we found some evidence for complex associations between mitochondrial nad5 $\Delta$ frequency and mitochondrial phenotypes, the combined results are consistent with the mitochondrial threshold effect theory, with phenotypic consequences arising only at high ( 50\%) nad5 levels. High-deletion level isolate HK105 (Fig. 2.1) consistently displayed phenotypes associated with mitochondrial dysfunction, including elevated ROS levels (Fig 2.3I, Fig 3.3B), distinct patterns of mitochondrial morphology (Fig 2.3), and rapid extinction rates during MA (Table 3.1). These results agree with previous work in C. briggsae that uncovered a trade-off between early fecundity and lifespan only when nad5 $\Delta$ frequency exceeded $\sim 40 \%$ (see Figure 3 Estes et al. 2011). Our results showing an increase in average nad5 $\Delta$ percentage up to a heteroplasmy level of $\sim 50 \%$ 
(Fig. 3.3A) also support the threshold effect theory, as well as recent findings suggesting that nad5 $\Delta$ behaves as a selfish element (Clark et al. 2012). Taken together, these results suggest that the maintenance of nad5 $\Delta$ heteroplasmy level within $C$. briggsae natural isolates appears to be under the control of at least two competing forces: a replicative advantage that promotes the accumulation of nad5 $\Delta$-bearing genomes up to the threshold level, at which point purifying selection against depressed mitochondrial function prevents further propagation. Confirmation of this hypothesis will require the simultaneous genotyping (e.g., through mitochondrial mRNA or DNA labeling) and phenotyping of individual mitochondria within the $C$. briggsae system. Additionally, we still lack a basic understanding of the mode(s) by which selection on individual mitochondria might act, including how and why particular mtDNA molecules are inherited. Such work will require the development of experimental methods for manipulating the size of the mitochondrial bottleneck or the strength of selection on mtDNA molecules. Together, such work would allow us to begin to understand the genetic and developmental mechanisms governing mitochondrial genotypic and phenotypic evolution, and would shed light on the forces controlling nad5 $\Delta$ heteroplasmy level.

After uncovering significant natural variation in mitochondrial phenotypes, we aimed to determine whether natural selection might be shaping the observed phenotypic variation. By reducing the power of natural selection and observing the evolution of traits, one is able to infer the strength of natural selection that is acting on 
these traits in nature. To reduce the effect of natural selection in the current study, we subjected lines of $C$. briggsae to extreme genetic drift for 20-50 generations and assayed ROS and heteroplasmy levels at three generational time points. Our work revealed a notable capacity for mitochondrial ROS evolution under extreme genetic drift, manifested in the significant change in ROS levels after only ten generations of inbreeding in three of the four isolates assayed. The pattern of evolution, however, was highly variable and strain-specific. ROS is often cited as a major destructive agent threatening genomic integrity and thereby wielding serious evolutionary implications. However, the results presented in Chapter five provide no evidence in support of a direct role for ROS in contributing to mitochondrial shape or population structure (Table 5.2) based on the lack of any correlation between ROS and other mitochondrial phenotypes. We also found no evidence for a vicious cycle of oxidative damage (Figs. $3.3 B, 4.1)$, since ROS was not correlated at all to measures of nuclear base substitution rate in Chapter four. Previous work also failed to identify a consistent relationship between ROS and aspects of mitochondrial shape and population structure (Koopman et al. 2005; Pletjushkina et al. 2006; Grünewald et al. 2010). However, the role of ROS in mutation accumulation is highly contentious, with a great deal of evidence supporting (Wei and Lee 2002; Busuttil et al. 2003, 2005; Wallace 2005b; Hiona and Leeuwenburgh 2008) and refuting (Jacobs 2003; Crabbe and Hill 2010) such an association. The above data suggest that ROS is not a major factor driving the accumulation of nuclear genomic base substitution mutations within the germline of Caenorhabditid nematodes. It is 
possible that the effects of oxidative stress will be more apparent in mtDNA mutational processes, or that ROS causes the accumulation of distinct mutational types in a tissuespecific manner; the examination of separate tissues and genomic rearrangements has proven fruitful (Dolle et al. 2000; Busuttil et al. 2005, 2007). However, the potential indirect mechanisms by which ROS may influence genomic stability and mutation accumulation represent numerous opportunities for future study. For example, recent work implicates ROS in mediating nuclear genome repair efficiency (Delsite et al. 2003; Rowe et al. 2008; Swartzlander et al. 2010). Further study examining which reactive species performs specific signaling functions, how signals are propagated from organelle to organismal levels, and what processes are affected will all need to be addressed to create a complete picture describing the role of ROS in mitochondrial and nuclear mutation accumulation.

It is becoming increasingly apparent that traits other than ROS are important for mitochondrial function. The findings reported in this thesis support a central role of $\Delta \Psi \mathrm{M}$ in governing mitochondrial shape and population structure and highlight the potential implications of $\triangle \Psi M$ variation in evolutionary adaptation and mitochondrial genome integrity. $\triangle \Psi \mathrm{M}$ not only displayed the greatest amount of among-isolate variation (Fig. 2.3), but also correlated significantly with several aspects of mitochondrial shape and population structure (Table 5.2). In Chapter Five, we identified the following three major patterns of relationship among mitochondrial form and function traits assayed here 1) mitochondria became bigger through elongation, 2) isolates with a 
more functional mitochondrial population (with relatively high overall $\Delta \Psi \mathrm{M}$ ) contained larger, more elongated mitochondria, and 3) isolates with a less functional mitochondrial population (with relatively low overall $\Delta \Psi \mathrm{M}$ ) contained a more heterogeneous mix of organelles. Using these bivariate relationships we developed a model in which overall $\triangle \Psi \mathrm{M}$ of the entire mitochondrial population influences organelle population structure and fusion-fission capacity (Fig 5.3). These results align well with previous results indicating that $\triangle \Psi \mathrm{M}$ plays an important role in mediating cycles of fusion-fission-apoptosis undergone by individual mitochondria (Ishihara et al. 2003; Mattenberger et al. 2003; Twig et al. 2008a,b), but adds a population-level perspective. Based on the model presented here, we would expect mitochondrial populations with lower $\triangle \Psi \mathrm{M}$ to have fewer mitochondria overall, a more heterogeneous population of organelles, and to participate in fewer fusion-fission cycles. Because $\triangle \Psi M$ and mitochondrial fusion-fission cycles may aid in mtDNA maintenance and integrity (Twig et al. 2008b; Kowald and Kirkwood 2011), such $\triangle \Psi M$ variation could also potentially contribute to intra- and interspecific differences in mtDNA mutation rates, heteroplasmy levels, and the ability to purge damaged genomes. Indeed, recent work shows that mitochondrial fusion is necessary for the removal of damaged mtDNA in $C$. elegans (Bess et al. 2012; Meyer and Bess 2012). These results present exciting new avenues for research into the potentially far-reaching and pleiotropic effects of $\Delta \Psi \mathrm{M}$ variation. In $C$. briggsae in particular, determining the role of mitochondrial $\triangle \Psi M$ and 
fusion-fission dynamics in controlling adaptation to varying thermal and oxygen conditions and the transmission of nad5 5 -bearing genomes will be enlightening.

The population level predictions of the model above are based on work done by Twig, et al. (2008) that shows that individual mitochondria undergo continuous cycles of fusion and fission. Twig and colleagues observed the spread of mitochondria-targeted photoactivatable GFP (mtPA-GFP) to all but a small subset of the mitochondrial population, estimating the $\sim 20$ individual mitochondria per cell do not undergo fusion events. Using TMRE (the uptake of which is dependent on $\Delta \Psi M$ ), they determined that these non-fusing mitochondria were less polarized than the rest of the mitochondrial population, and by following individual mitochondria after fusion, they were able to observe that fission events followed fusion events. By double-labeling mitochondria with TMRE and mTPA-GFP they observed that the two daughter mitochondria that result from a fission event have opposite deflections in $\Delta \Psi \mathrm{M}$ in $85 \%$ of fusion-fission events, with one daughter organelle experiencing increased $\Delta \Psi M$ and the other experiencing decreased $\triangle \Psi \mathrm{M}$. After tracking the fate of individual mitochondria, they observed that the depolarized daughter mitochondrion was less likely to undergo subsequent fusion events. The researchers also performed immunoreactivity assays on fixed cells to quantify the relative amount of the fusion protein OPA1 present within individual mitochondria. They found that non-fusing mitochondria contained less OPA1 than fusion-capable mitochondria. Finally, following inhibition of proteolytic activity to prevent degradation of mitochondria within autophagosomes, Twig, et al. observed that 
the mitochondria within autophagosomes contained reduced OPA1 compared to mitochondria outside autophagosomes. Taken together, this work is highly suggestive that mitochondria undergo paired fusion-fission cycles that result in two daughter organelles with distinct $\Delta \Psi \mathrm{M}$. The depolarized mitochondrion is less likely to undergo subsequent fusion and more likely to undergo autophagy. While this work has yet to be thoroughly tested, our data showing that isolates with lower $\triangle \Psi \mathrm{M}$ have smaller, more heterogeneous mitochondrial populations are in line with the work presented in Twig et al. (2008).

\section{The Future of Mitochondrial Study}

The current study represents a first step toward developing our understanding of mitochondria as a population of individuals, rather than describing the behavior of individual mitochondrion. Given the recent work suggesting that mitochondria constantly undergo cycles of fusion and fission (Twig et al. 2008a), potentially mixing metabolites and even genomes, it is becoming increasingly apparent that treating mitochondria as distinct units is inappropriate. Rather, significant effort should be put into describing the dynamics and genetics of mitochondrial populations. It has been proposed that the observed heterogeneity of mitochondrial function, shape, and distribution may determine what role a mitochondrion will play within the greater population (Kuznetsov and Margreiter 2009). It seems likely that understanding such population level relationships will further explain the substantial differences in cellular/tissue responses to mtDNA mutation heteroplasmy. The existence of such 
heterogeneity, though nearly completely overlooked, will almost certainly influence the conclusions and implications of a great deal of the mitochondrial studies completed to this date. For instance, if different functional populations of mitochondria exist that maintain distinct characteristics (such as $\triangle \Psi \mathrm{M}$ levels or ROS production rates) based on their activity within the cell, the entire nature of the model presented in Chapter five will be altered. Despite the potential importance of such basic knowledge of mitochondrial behavior, we have basically no experimental information describing such features of the mitochondrial population, and no work suggesting a path forward to assay or describe the different roles mitochondria may play. One reason that such little progress has been made in terms of understanding mitochondrial population-level traits is the difficulty in quantifying mitochondrial phenotypes. Even for relatively simple to measure traits such as ROS and $\triangle \Psi M$ there are substantial and undeniable issues with quantification, including comparing values across experiments and especially from different labs, even when using very similar practices. Mitochondria are highly responsive to the environment and have evolved to maintain energetic homeostasis despite significant alterations to a many environmental variables (Hochachka and Somero 2002). Thus, even small changes in humidity, light availability, oxygen tension, and other factors may induce shifts in mitochondrial function that will be difficult to control for between studies. All of these shortcomings highlight the fact that mitochondrial research is still in need of basic work. 
Recent advancements in microscopic imaging and fluorescent probes have significantly improved our ability to monitor mitochondrial in vivo. However, several aspects still require further development. For instance, the extent to which fluorescent probes interfere with normal mitochondrial function and form are relatively untested and should be understood. Additionally, the way in which mitochondrial morphology is assayed varies substantially among different studies. For instance, the current study was only able to examine the $2 \mathrm{D}$ structure of individual mitochondria. This means that mitochondria occupying a different plane than the plane being assayed may appear as a series of punctate organelles, while in actuality it might exist as an elongated tube. Further, this study was only able to differentiate between mitochondria stained with mainly MitoTracker Red CMXRos or MitoTracker Green FM as determined by images taken with red and green filters. We could not, however, look at the relative fluorescence of each individual functional mitochondrion because of the processing steps involved in the morphological analysis. Work similar in scope to the current study will benefit greatly from the use of state-of-the-art image analysis software that creates accurate 3D renderings of images while retaining all of the fluorescent and morphological information for each mitochondrion. This software, combined with fluorescent in situ hybridization of heteroplasmic genomes within mitochondria have the potential to provide unprecedented information about how heteroplasmy levels influence individual mitochondrial function and shape, directly testing the idea of threshold effects in individual organelles. 
More generally, the work presented in this thesis indicates that experimental evolutionary approaches combined with fluorescent microscopy holds promise for developing our understanding of important mitochondrial phenotypes and other celllevel processes. While evolutionary and cellular biology have provided important insights into genetic and cellular processes respectively, these two realms of biology rarely overlap. Evolutionary cell biology has historically been overlooked and is only now emerging as a focused area of research in need of both theoretical and empirical work. Three of the four studies presented above represent the first of their kind, combining assays of subcellular phenotypes and evolutionary biology. Because mitochondria are essential for organismal health and survival mitochondrial genotypic and phenotypic variation can ultimately produce variation in organismal fitness. Thus, mitochondrial research is uniquely suited for work focused on evolutionary cell biology. In addition to the future avenues for experimentation listed above, further work in this discipline specific to mitochondria include: describing the role of mitochondrial fission-fusionapoptosis cycles in maintaining mtDNA genome integrity, determining what evolutionary and cell-biological factors influence the efficacy of such a system, examining the relative strengths of evolutionary forces acting upon mitochondria, and investigating the extent to which epistasis between nuclear and mtDNA genes influence mitochondrial function and organismal fitness. Its amenability to evolutionary experimentation and in vivo cell biological studies suggest that Caenorhabditid 
nematodes will prove an invaluable model system for advancing the field of evolutionary cell biology. 


\section{REFERENCES}

Adachi, H., Y. Fujiwara and N. Ishii. 1998. Effects of Oxygen on Protein Carbonyl and Aging in Caenorhabditis elegans Mutants With Long (age-1) and Short (mev-1) Life Spans. The Journals of Gerontology Series A: Biological Sciences and Medical Sciences 53A:B240-B244.

Aitken, R. J. 1995. Free radicals, lipid peroxidation and sperm function. Reprod Fertil Dev 7:659-668.

Aitken, R. J., G. N. De luliis and R. I. McLachlan. 2009. Biological and clinical significance of DNA damage in the male germ line. International journal of andrology 32:46-56.

Ajie, B. C., S. Estes, M. Lynch and P. C. Phillips. 2005. Behavioral degradation under mutation accumulation in Caenorhabditis elegans. Genetics 170:655-60.

Albertson, D. G. and J. N. Thomson. 1976. The pharynx of Caenorhabditis elegans. Philos Trans R Soc Lond B Biol Sci 275:299-325.

Allee, W. C. 1931. Co-Operation Among Animals. American Journal of Sociology 37:386398.

An, J. H., K. Vranas, M. Lucke, H. Inoue, N. Hisamoto, K. Matsumoto and T. K. Blackwell. 2005. Regulation of the Caenorhabditis elegans oxidative stress defense protein SKN-1 by glycogen synthase kinase-3. Proc Natl Acad Sci U S A 102:16275-16280.

Arczewska, K. D., K. Michalickova, I. M. Donaldson and H. Nilsen. 2008. The contribution of DNA base damage to human cancer is modulated by the base excision repair interaction network. Critical reviews in oncogenesis 14:217-73.

Arnqvist, G., D. K. Dowling, P. Eady, L. Gay, T. Tregenza, M. Tuda and D. J. Hosken. 2010. Genetic architecture of metabolic rate: environment specific epistasis between mitochondrial and nuclear genes in an insect. Evolution 64:3354-3363.

Baer, C. F., F. Shaw, C. Steding, M. Baumgartner, A. Hawkins, A. Houppert, N. Mason, M. Reed, K. Simonelic, W. Woodard and M. Lynch. 2005. Comparative evolutionary genetics of spontaneous mutations affecting fitness in rhabditid nematodes. Proc Natl Acad Sci U S A 102:5785-5790. 
Balaban, R. S., S. Nemoto and T. Finkel. 2005. Mitochondria, oxidants, and aging. Cell 120:483-495.

Ballard, J. W. O. and R. G. Melvin. 2010. Linking the mitochondrial genotype to the organismal phenotype. Mol Ecol 19:1523-39.

Ballard, J. W. and M. C. Whitlock. 2004. The incomplete natural history of mitochondria. Mol Ecol 13:729-744.

Bandy, B. and A. J. Davison. 1990. Mitochondrial mutations may increase oxidative stress: implications for carcinogenesis and aging? Free Radic Biol Med 8:523-539.

Barnes, D. E. and T. Lindahl. 2004. Repair and genetic consequences of endogenous DNA base damage in mammalian cells. Annual review of genetics 38:445-76.

Bazin, E., S. Glemin and N. Galtier. 2006. Population size does not influence mitochondrial genetic diversity in animals. Science 312:570-572.

Beehler, B. C., J. Przybyszewski, H. B. Box and M. F. Kulesz-Martin. 1992. Formation of 8hydroxydeoxyguanosine within DNA of mouse keratinocytes exposed in culture to UVB and $\mathrm{H} 2 \mathrm{O}$ 2. Carcinogenesis 13:2003-2007.

Berquist, B. R. and D. M. Wilson. 2012. Pathways for repairing and tolerating the spectrum of oxidative DNA lesions. Cancer letters null.

Bess, A. S., T. L. Crocker, I. T. Ryde and J. N. Meyer. 2012. Mitochondrial dynamics and autophagy aid in removal of persistent mitochondrial DNA damage in Caenorhabditis elegans. Nucleic acids research, doi: 10.1093/nar/gks532.

Biskup, S. and D. J. Moore. 2006. Detrimental deletions: mitochondria, aging and Parkinson's disease. BioEssays : news and reviews in molecular, cellular and developmental biology 28:963-7.

Blackstone, N. W. 2009. Is Evolutionary Theory Central to Molecular Cell Biology? The Journal of the Evolutionary Studies Consortium 1:34-43.

Bossy-Wetzel, E., M. J. Barsoum, A. Godzik, R. Schwarzenbacher and S. A. Lipton. 2003. Mitochondrial fission in apoptosis, neurodegeneration and aging. Current Opinion in Cell Biology 15:706-716. 
Brand, M. D. 2000. Uncoupling to survive? The role of mitochondrial inefficiency in ageing. Exp Gerontol 35:811-820.

Burton, R. S., R. J. Byrne and P. D. Rawson. 2007. Three divergent mitochondrial genomes from California populations of the copepod Tigriopus californicus. Gene 403:53-9.

Burton, R. S., C. K. Ellison and J. S. Harrison. 2006. The sorry state of F2 hybrids: consequences of rapid mitochondrial DNA evolution in allopatric populations. The American naturalist 168 Suppl :S14-24.

Busuttil, R. A., A. M. Garcia, C. Cabrera, A. Rodriguez, Y. Suh, W. H. Kim, T. T. Huang and J. Vijg. 2005. Organ-specific increase in mutation accumulation and apoptosis rate in CuZn-superoxide dismutase-deficient mice. Cancer research 65:11271-5.

Busuttil, R. A., A. M. Garcia, R. L. Reddick, M. E. T. Dollé, R. B. Calder, J. F. Nelson and J. Vijg. 2007. Intra-organ variation in age-related mutation accumulation in the mouse. PloS one 2:e876. Public Library of Science.

Busuttil, R. A., M. Rubio, M. E. T. Dollé, J. Campisi and J. Vijg. 2003. Oxygen accelerates the accumulation of mutations during the senescence and immortalization of murine cells in culture. Aging Cell 2:287-294.

Chan, D. C. 2006a. Mitochondria: dynamic organelles in disease, aging, and development. Cell 125:1241-1252.

Chan, D. C. 2006b. Mitochondrial fusion and fission in mammals. Annu Rev Cell Dev Biol 22:79-99.

Chen, H. and D. C. Chan. 2005. Emerging functions of mammalian mitochondrial fusion and fission. Hum Mol Genet 14 Spec No:R283-9.

Chen, H., A. Chomyn and D. C. Chan. 2005. Disruption of fusion results in mitochondrial heterogeneity and dysfunction. J Biol Chem 280:26185-26192.

Chen, H., J. M. McCaffery and D. C. Chan. 2007. Mitochondrial fusion protects against neurodegeneration in the cerebellum. Cell 130:548-562.

Chen, H., M. Vermulst, Y. E. Wang, A. Chomyn, T. A. Prolla, J. M. McCaffery and D. C. Chan. 2010. Mitochondrial fusion is required for mtDNA stability in skeletal muscle and tolerance of mtDNA mutations. Cell 141:280-289. 
Chen, Q., S. Moghaddas, C. L. Hoppel and E. J. Lesnefsky. 2008. Ischemic defects in the electron transport chain increase the production of reactive oxygen species from isolated rat heart mitochondria. Am J Physiol Cell Physiol 294:C460-6.

Cheng, K. C., D. S. Cahill, H. Kasai, S. Nishimura and L. A. Loeb. 1992. 8-Hydroxyguanine, an abundant form of oxidative DNA damage, causes G----T and A----C substitutions. The Journal of biological chemistry 267:166-72.

Chinnery, P. F., M. A. Johnson, T. M. Wardell, R. Singh-Kler, C. Hayes, D. T. Brown, R. W. Taylor, L. A. Bindoff and D. M. Turnbull. 2000. The epidemiology of pathogenic mitochondrial DNA mutations. Ann Neurol 48:188-193.

Clark, K. A., D. K. Howe, K. Gafner, D. Kusuma, S. Ping, S. Estes and D. R. Denver. 2012. Selfish little circles: transmission bias and evolution of large deletionbearingmitochondrial DNA in Caenorhabditis briggsae nematodes. PLoS One 7:e41433.

Crabbe, R. A. and K. A. Hill. 2010. Heart tissue of harlequin (hq)/Big Blue mice has elevated reactive oxygen species without significant impact on the frequency and nature of point mutations in nuclear DNA. Mutation research 691:64-71.

Croteau, D. L. 1997. Repair of Oxidative Damage to Nuclear and Mitochondrial DNA in Mammalian Cells. Journal of Biological Chemistry 272:25409-25412.

Cutter, A. D., M. A. Félix, A. Barriere and D. Charlesworth. 2006. Patterns of nucleotide polymorphism distinguish temperate and tropical wild isolates of Caenorhabditis briggsae. Genetics 173:2021-2031.

Cutter, A. D., W. Yan, N. Tsvetkov, S. Sunil and M. A. Felix. 2010. Molecular population genetics and phenotypic sensitivity to ethanol for a globally diverse sample of the nematode Caenorhabditis briggsae. Mol Ecol 19:798-809.

David, S. S., V. L. O'Shea and S. Kundu. 2007. Base-excision repair of oxidative DNA damage. Nature 447:941-50.

Delsite, R. L., L. J. Rasmussen, A. K. Rasmussen, A. Kalen, P. C. Goswami and K. K. Singh. 2003. Mitochondrial impairment is accompanied by impaired oxidative DNA repair in the nucleus. Mutagenesis 18:497-503.

Denver, D. R., P. C. Dolan, L. J. Wilhelm, W. Sung, J. I. Lucas-Lledo, D. K. Howe, S. C. Lewis, K. Okamoto, W. K. Thomas, M. Lynch and C. F. Baer. 2009. A genome-wide view 
of Caenorhabditis elegans base-substitution mutation processes. Proc Natl Acad Sci U S A 106:16310-16314.

Denver, D. R., S. Feinberg, C. Steding, M. Durbin and M. Lynch. 2006. The relative roles of three DNA repair pathways in preventing Caenorhabditis elegans mutation accumulation. Genetics 174:57-65.

Denver, D. R., K. Morris, M. Lynch, L. L. Vassilieva and W. K. Thomas. 2000. High direct estimate of the mutation rate in the mitochondrial genome of Caenorhabditis elegans. Science 289:2342-2344.

Denver, D. R., K. Morris and W. K. Thomas. 2003a. Phylogenetics in Caenorhabditis elegans: an analysis of divergence and outcrossing. Mol Biol Evol 20:393-400.

Denver, D. R., S. L. Swenson and M. Lynch. 2003b. An evolutionary analysis of the helixhairpin-helix superfamily of DNA repair glycosylases. Molecular Biology and Evolution 20:1603-1611.

Denver, D. R., L. J. Wilhelm, D. K. Howe, K. Gafner, P. C. Dolan and C. F. Baer. 2012. Variation in base-substitution mutation in experimental and natural lineages of Caenorhabditis nematodes. Genome Biol Evol 4:513-22.

Diaz, F., M. P. Bayona-Bafaluy, M. Rana, M. Mora, H. Hao and C. T. Moraes. 2002. Human mitochondrial DNA with large deletions repopulates organelles faster than fulllength genomes under relaxed copy number control. Nucleic Acids Res 30:4626-4633.

Dimmer, K. S., S. Fritz, F. Fuchs, M. Messerschmitt, N. Weinbach, W. Neupert and B. Westermann. 2002. Genetic basis of mitochondrial function and morphology in Saccharomyces cerevisiae. Mol Biol Cell 13:847-853.

Dingley, S., E. Polyak, R. Lightfoot, J. Ostrovsky, M. Rao, T. Greco, H. Ischiropoulos and M. J. Falk. 2009. Mitochondrial respiratory chain dysfunction variably increases oxidant stress in Caenorhabditis elegans. Mitochondrion 10:125-36.

Dolle, M. E. T., W. K. Snyder, J. A. Gossen, P. H. M. Lohman and J. Vijg. 2000. Distinct spectra of somatic mutations accumulated with age in mouse heart and small intestine. Proceedings of the National Academy of Sciences 97:8403-8408.

Dowling, D. K. and L. W. Simmons. 2009. Reactive oxygen species as universal constraints in life-history evolution. Proceedings. Biological sciences / The Royal Society 276:1737-1745. Proceedings of the Royal Society Biological Sciences. 
Drake, J. W., B. Charlesworth, D. Charlesworth and J. F. Crow. 1998. Rates of spontaneous mutation. Genetics 148:1667-86.

Droge, W. 2002. Free Radicals in the Physiological Control of Cell Function. Physiol Rev 82:47-95.

Durieux, J., S. Wolff and A. Dillin. 2011. The cell-non-autonomous nature of electron transport chain-mediated longevity. Cell 144:79-91.

Duvezin-Caubet, S., R. Jagasia, J. Wagener, S. Hofmann, A. Trifunovic, A. Hansson, A. Chomyn, M. F. Bauer, G. Attardi, N. G. Larsson, W. Neupert and A. S. Reichert. 2006. Proteolytic processing of OPA1 links mitochondrial dysfunction to alterations in mitochondrial morphology. J Biol Chem 281:37972-37979.

Ellison, C. K. and R. S. Burton. 2010. Cytonuclear conflict in interpopulation hybrids: the role of RNA polymerase in mtDNA transcription and replication. J Evol Biol 23:528-538.

Ellison, C. K. and R. S. Burton. 2006. Disruption of mitochondrial function in interpopulation hybrids of Tigriopus californicus. Evolution 60:1382-1391.

Ellison, C. K., O. Niehuis and J. Gadau. 2008. Hybrid breakdown and mitochondrial dysfunction in hybrids of Nasonia parasitoid wasps. J Evol Biol 21:1844-1851.

Estes, S., B. Ajie, M. Lynch and P. Phillips. 2005. Spontaneous mutational correlations for life-history, morphological and behavioral characters in Caenorhabditis elegans. Genetics 170:645-53.

Estes, S., A. L. Coleman-Hulbert, K. A. Hicks, G. de Haan, S. R. Martha, J. B. Knapp, S. W. Smith, K. C. Stein and D. R. Denver. 2011. Natural variation in life history and aging phenotypes is associated with mitochondrial DNA deletion frequency in Caenorhabditis briggsae. BMC Evol Biol 11:11.

Falk, M. J., Z. Zhang, J. R. Rosenjack, I. Nissim, E. Daikhin, M. M. Sedensky, M. Yudkoff and P. G. Morgan. 2008. Metabolic pathway profiling of mitochondrial respiratory chain mutants in C. elegans. Molecular genetics and metabolism 93:388-97.

Fangue, N. A., J. G. Richards and P. M. Schulte. 2009. Do mitochondrial properties explain intraspecific variation in thermal tolerance? The Journal of experimental biology 212:514-22. 
Fensgård, $\varnothing$., H. Kassahun, I. Bombik, T. Rognes, J. M. Lindvall and H. Nilsen. 2010. A two-tiered compensatory response to loss of DNA repair modulates aging and stress response pathways. Aging 2:133-59.

Floyd, R. A., M. S. West, K. L. Eneff, W. E. Hogsett and D. T. Tingey. 1988. Hydroxyl free radical mediated formation of 8-hydroxyguanine in isolated DNA. Archives of Biochemistry and Biophysics 262:266-272.

Fontaine, K. M., J. R. Cooley and C. Simon. 2007. Evidence for paternal leakage in hybrid periodical cicadas (Hemiptera: Magicicada spp.). PloS one 2:e892. Public Library of Science.

Fortune, M. T. 2000. Dramatic, expansion-biased, age-dependent, tissue-specific somatic mosaicism in a transgenic mouse model of triplet repeat instability. Human Molecular Genetics 9:439-445.

Garcia, A. M., R. B. Calder, M. E. T. Dollé, M. Lundell, P. Kapahi and J. Vijg. 2010. Ageand temperature-dependent somatic mutation accumulation in Drosophila melanogaster. PLoS genetics 6:e1000950. Public Library of Science.

Garcia, A. M., A. Derventzi, R. A. Busuttil, R. B. Calder, E. Perez, L. Chadwell, M. E. T. Dollé, M. Lundell and J. Vijg. 2007. A model system for analyzing somatic mutations in Drosophila melanogaster. Nature methods 4:401-3.

Gaskova, D., A. DeCorby and B. D. Lemire. 2007. DiS-C3(3) monitoring of in vivo mitochondrial membrane potential in C. elegans. Biochem Biophys Res Commun 354:814-819.

Gershoni, M., A. R. Templeton and D. Mishmar. 2009. Mitochondrial bioenergetics as a major motive force of speciation. BioEssays : news and reviews in molecular, cellular and developmental biology 31:642-650.

Gotelli, N. J. and A. M. Ellison. 2004. A Primer of Ecological Genetics. Sinauer Associates, Inc., Sunderland.

Grad, L. I. and B. D. Lemire. 2004. Mitochondrial complex I mutations in Caenorhabditis elegans produce cytochrome c oxidase deficiency, oxidative stress and vitaminresponsive lactic acidosis. Hum Mol Genet 13:303-314. 
Grad, L. I., L. C. Sayles and B. D. Lemire. 2005. Introduction of an additional pathway for lactate oxidation in the treatment of lactic acidosis and mitochondrial dysfunction in Caenorhabditis elegans. Proc Natl Acad Sci U S A 102:18367-18372.

Graef, M. and J. Nunnari. 2011. Mitochondria regulate autophagy by conserved signalling pathways. EMBO J 30:2101-2114.

Griffin, E. E. and D. C. Chan. 2006. Domain interactions within Fzo1 oligomers are essential for mitochondrial fusion. J Biol Chem 281:16599-16606.

Gruber, J., L. F. Ng, S. Fong, Y. T. Wong, S. A. Koh, C.-B. Chen, G. Shui, W. F. Cheong, S. Schaffer, M. R. Wenk and B. Halliwell. 2011. Mitochondrial changes in ageing Caenorhabditis elegans--what do we learn from superoxide dismutase knockouts? PloS one 6:e19444. Public Library of Science.

Gruber, J., S. Schaffer and B. Halliwell. 2008. The mitochondrial free radical theory of ageing--where do we stand? Front Biosci 13:6554-6579.

Grünewald, A., L. Voges, A. Rakovic, M. Kasten, H. Vandebona, C. Hemmelmann, K. Lohmann, S. Orolicki, A. Ramirez, A. H. V. Schapira, P. P. Pramstaller, C. M. Sue and C. Klein. 2010. Mutant Parkin impairs mitochondrial function and morphology in human fibroblasts. PloS one 5:e12962. Public Library of Science.

Gupta, B. P., R. Johnsen and N. Chen. 2007. Genomics and biology of the nematode Caenorhabditis briggsae. P. doi/10.1895/wormbook.1.136.1, http://www.wormbook. in WormBook, ed. WormBook. The C. elegans Research Community, WormBook.

Haag-Liautard, C., N. Coffey, D. Houle, M. Lynch, B. Charlesworth and P. D. Keightley. 2008. Direct estimation of the mitochondrial DNA mutation rate in Drosophila melanogaster. PLoS Biol 6:e204.

Haas, R. H. 2010. Autism and mitochondrial disease. Dev Disabil Res Rev 16:144-153.

Halligan, D. L. and P. D. Keightley. 2009. Spontaneous Mutation Accumulation Studies in Evolutionary Genetics. Annual Review of Ecology, Evolution, and Systematics 40:151172. Annual Reviews.

Halliwell, B. and J. Gutteridge. 2007. Free Radicals in Biology and Medicine. 4th ed. Oxford University Press, Oxford. 
Harman, D. 1956. Aging: a theory based on free radical and radiation chemistry. J Gerontol 11:298-300.

Hartman, P. S., N. Ishii, E. B. Kayser, P. G. Morgan and M. M. Sedensky. 2001. Mitochondrial mutations differentially affect aging, mutability and anesthetic sensitivity in Caenorhabditis elegans. Mechanisms of Ageing and Development 122:1187-1201.

Hasty, P., J. Campisi, J. Hoeijmakers, H. van Steeg and J. Vijg. 2003. Aging and genome maintenance: lessons from the mouse? Science (New York, N.Y.) 299:1355-9.

Helbock, H. J., K. B. Beckman, M. K. Shigenaga, P. B. Walter, A. A. Woodall, H. C. Yeo and B. N. Ames. 1998. DNA oxidation matters: the HPLC-electrochemical detection assay of 8-oxo-deoxyguanosine and 8-oxo-guanine. Proceedings of the National Academy of Sciences of the United States of America 95:288-93.

Hicks, K. A., D. K. Howe, A. Leung, D. R. Denver and S. Estes. 2012. In Vivo Quantification Reveals Extensive Natural Variation in Mitochondrial Form and Function in Caenorhabditis briggsae. PLoS ONE 7:e43837. Public Library of Science.

Hiona, A. and C. Leeuwenburgh. 2008. The role of mitochondrial DNA mutations in aging and sarcopenia: implications for the mitochondrial vicious cycle theory of aging. Exp Gerontol 43:24-33.

Hochachka, P. and G. Somero. 2002. Biochemical Adaptation: Mechanism and Process in Physiological Evolution. Oxford University Press, New York.

Howe, D. K., C. F. Baer and D. R. Denver. 2010. High rate of large deletions in Caenorhabditis briggsae mitochondrial genome mutation processes. Genome Biology and Evolution 2010:29-38.

Howe, D. K. and D. R. Denver. 2008. Muller's Ratchet and compensatory mutation in Caenorhabditis briggsae mitochondrial genome evolution. BMC Evol Biol 8:62.

Hsu, G. W., M. Ober, T. Carell and L. S. Beese. 2004. Error-prone replication of oxidatively damaged DNA by a high-fidelity DNA polymerase. Nature 431:217-21. Nature Publishing Group.

Huang, C., C. Xiong and K. Kornfeld. 2004. Measurements of age-related changes of physiological processes that predict lifespan of Caenorhabditis elegans. Proc Natl Acad Sci U S A 101:8084-8089. 
Hussain, S. P., K. Raja, P. A. Amstad, M. Sawyer, L. J. Trudel, G. N. Wogan, L. J. Hofseth, P. G. Shields, T. R. Billiar, C. Trautwein, T. Hohler, P. R. Galle, D. H. Phillips, R. Markin, A. J. Marrogi and C. C. Harris. 2000. Increased p53 mutation load in nontumorous human liver of wilson disease and hemochromatosis: oxyradical overload diseases. Proceedings of the National Academy of Sciences of the United States of America 97:12770-5.

Hyde, B. B., G. Twig and O. S. Shirihai. 2010. Organellar vs cellular control of mitochondrial dynamics. Seminars in cell \& developmental biology 21:575-81.

Ichishita, R., K. Tanaka, Y. Sugiura, T. Sayano, K. Mihara and T. Oka. 2008. An RNAi screen for mitochondrial proteins required to maintain the morphology of the organelle in Caenorhabditis elegans. J Biochem 143:449-454.

lenco, E. C., C. Simoncini, D. Orsucci, L. Petrucci, M. Filosto, M. Mancuso and G. Siciliano. 2011. May "mitochondrial eve" and mitochondrial haplogroups play a role in neurodegeneration and Alzheimer's disease? Int J Alzheimers Dis 2011:709061.

Imlay, J. A. 2008. Cellular defenses against superoxide and hydrogen peroxide. Annual review of biochemistry 77:755-76. NIH Public Access.

Inoue, H., N. Hisamoto, J. H. An, R. P. Oliveira, E. Nishida, T. K. Blackwell and K. Matsumoto. 2005. The C. elegans p38 MAPK pathway regulates nuclear localization of the transcription factor SKN-1 in oxidative stress response. Genes Dev 19:2278-2283.

Irrcher, I., H. Aleyasin, E. L. Seifert, S. J. Hewitt, S. Chhabra, M. Phillips, A. K. Lutz, M. W. Rousseaux, L. Bevilacqua, A. Jahani-Asl, S. Callaghan, J. G. MacLaurin, K. F. Winklhofer, P. Rizzu, P. Rippstein, R. H. Kim, C. X. Chen, E. A. Fon, R. S. Slack, M. E. Harper, H. M. McBride, T. W. Mak and D. S. Park. 2010. Loss of the Parkinson's disease-linked gene DJ1 perturbs mitochondrial dynamics. Hum Mol Genet 19:3734-3746.

Iser, W. B., D. Kim, E. Bachman and C. Wolkow. 2005. Examination of the requirement for ucp-4, a putative homolog of mammalian uncoupling proteins, for stress tolerance and longevity in C. elegans. Mechanisms of ageing and development 126:1090-6.

Ishihara, N., A. Jofuku, Y. Eura and K. Mihara. 2003. Regulation of mitochondrial morphology by membrane potential, and DRP1-dependent division and FZO1dependent fusion reaction in mammalian cells. Biochem Biophys Res Commun 301:891898.

Ishizawa, M., Y. Kobayashi, T. Miyamura and S. Matsuura. 1991. Simple procedure of DNA isolation from human serum. Nucleic acids research 19:5792. 
Jacobs, H. T. 2003. The mitochondrial theory of aging: dead or alive? Aging Cell 2:11-17.

Jagasia, R., P. Grote, B. Westermann and B. Conradt. 2005. DRP-1-mediated mitochondrial fragmentation during EGL-1-induced cell death in C. elegans. Nature 433:754-760.

Janssen, R. J., L. G. Nijtmans, L. P. van den Heuvel and J. A. Smeitink. 2006.

Mitochondrial complex I: structure, function and pathology. J Inherit Metab Dis 29:499515.

Jones, A. G., S. J. Arnold and R. Burger. 2007. The mutation matrix and the evolution of evolvability. Evolution; international journal of organic evolution 61:727-745.

Jovelin, R. and A. D. Cutter. 2011. MicroRNA sequence variation potentially contributes to within-species functional divergence in the nematode Caenorhabditis briggsae. Genetics 189:967-76.

Joyner-Matos, J., L. C. Bean, H. L. Richardson, T. Sammeli and C. F. Baer. 2011. No evidence of elevated germline mutation accumulation under oxidative stress in Caenorhabditis elegans. Genetics 189:1439-47.

Kageyama, Y., Z. Zhang and H. Sesaki. 2011. Mitochondrial division: molecular machinery and physiological functions. Current opinion in cell biology 23:427-34.

Kasai, H. 2002. Chemistry-based studies on oxidative DNA damage: formation, repair, and mutagenesis. Free radical biology \& medicine 33:450-6.

Kasai, H. and S. Nishimura. 1984. Hydroxylation of deoxyguanosine at the C-8 position by ascorbic acid and other reducing agents. Nucleic acids research 12:2137-45.

Kasai, H. and S. Nishimura. 1986. Hydroxylation of guanine in nucleosides and DNA at the $\mathrm{C}-8$ position by heated glucose and oxygen radical-forming agents. Environmental health perspectives 67:111-6.

Kayser, E.-B., M. M. Sedensky and P. G. Morgan. 2004. The effects of complex I function and oxidative damage on lifespan and anesthetic sensitivity in Caenorhabditis elegans. Mechanisms of ageing and development 125:455-64.

Keightley, P. D., U. Trivedi, M. Thomson, F. Oliver, S. Kumar and M. L. Blaxter. 2009. Analysis of the genome sequences of three Drosophila melanogaster spontaneous mutation accumulation lines. Genome Res 19:1195-201. 
Keller, I. and O. Seehausen. 2012. Thermal adaptation and ecological speciation. Molecular ecology 21:782-99.

Khrapko, K. and J. Vijg. 2009. Mitochondrial DNA mutations and aging: devils in the details? Trends Genet 25:91-98.

Kirkwood, T. B. L. 2005. Understanding the odd science of aging. Cell 120:437-47.

Klaunig, J. E. and L. M. Kamendulis. 2004. THE ROLE OF OXIDATIVE STRESS IN CARCINOGENESIS. Annual Reviews.

Knott, A. B. and E. Bossy-Wetzel. 2008. Impairing the mitochondrial fission and fusion balance: a new mechanism of neurodegeneration. Annals of the New York Academy of Sciences 1147:283-92.

Koboldt, D. C., J. Staisch, B. Thillainathan, K. Haines, S. E. Baird, H. M. Chamberlin, E. S. Haag, R. D. Miller and B. P. Gupta. 2010. A toolkit for rapid gene mapping in the nematode Caenorhabditis briggsae. BMC genomics 11:236.

Kondrashov, F. A. and A. S. Kondrashov. 2010. Measurements of spontaneous rates of mutations in the recent past and the near future. Phil Trans R Soc B 365:1169-76.

Koopman, W. J., S. Verkaart, H. J. Visch, F. H. van der Westhuizen, M. P. Murphy, L. W. van den Heuvel, J. A. Smeitink and P. H. Willems. 2005. Inhibition of complex I of the electron transport chain causes O2-. - mediated mitochondrial outgrowth. Am J Physiol Cell Physiol 288:C1440-50.

Koopman, W. J., H. J. Visch, J. A. M. Smeitink and P. H. G. M. Willems. 2006. Simultaneous quantitative measurement and automated analysis of mitochondrial morphology, mass, potential, and motility in living human skin fibroblasts. Cytometry Part A 69A:1-12. Wiley Subscription Services, Inc., A Wiley Company.

Kowald, A. and T. B. L. Kirkwood. 1996. A network theory of ageing: the interactions of defective mitochondria, aberrant proteins, free radicals and scavengers in the ageing process. Mutation Research/DNAging 316:209-236.

Kowald, A. and T. B. L. Kirkwood. 2011. Evolution of the mitochondrial fusion-fission cycle and its role in aging. Proc Natl Acad Sci U S A 108:10237-42. 
Kuznetsov, A. V., I. Kehrer, A. V. Kozlov, M. Haller, H. Redl, M. Hermann, M. Grimm and J. Troppmair. 2011. Mitochondrial ROS production under cellular stress: comparison of different detection methods. Anal Bioanal Chem 400:2383-2390.

Kuznetsov, A. V. and R. Margreiter. 2009. Heterogeneity of Mitochondria and Mitochondrial Function within Cells as Another Level of Mitochondrial Complexity. Int J Mol Sci 10:1911-1929.

Labrousse, A. M., M. D. Zappaterra, D. A. Rube and A. M. van der Bliek. 1999. C. elegans dynamin-related protein DRP-1 controls severing of the mitochondrial outer membrane. Mol Cell 4:815-826.

Lee, S. J., A. B. Hwang and C. Kenyon. 2010. Inhibition of respiration extends C. elegans life span via reactive oxygen species that increase HIF-1 activity. Current biology : CB 20:2131-6.

Lee, S. S., R. Y. Lee, A. G. Fraser, R. S. Kamath, J. Ahringer and G. Ruvkun. 2003. A systematic RNAi screen identifies a critical role for mitochondria in C. elegans longevity. Nature Genetics 33:40-48.

Lee, Y. J., S. Y. Jeong, M. Karbowski, C. L. Smith and R. J. Youle. 2004. Roles of the mammalian mitochondrial fission and fusion mediators Fis1, Drp1, and Opa1 in apoptosis. Molecular biology of the cell 15:5001-11.

Legros, F., A. Lombès, P. Frachon and M. Rojo. 2002. Mitochondrial fusion in human cells is efficient, requires the inner membrane potential, and is mediated by mitofusins. Molecular biology of the cell 13:4343-54.

Lemire, B. D., M. Behrendt, A. DeCorby and D. Gaskova. 2009. C. elegans longevity pathways converge to decrease mitochondrial membrane potential. Mechanisms of Ageing and Development 130:461-465.

Lenaz, G., R. Fato, M. L. Genova, C. Bergamini, C. Bianchi and A. Biondi. 2006. Mitochondrial Complex I: structural and functional aspects. Biochim Biophys Acta 1757:1406-1420.

Lewis, J. A., C. H. Wu, J. H. Levine and H. Berg. 1980. Levamisole-resistant mutants of the nematode Caenorhabditis elegans appear to lack pharmacological acetylcholine receptors. Neuroscience 5:967-989. 
Lewis, S. E. M. and R. J. Aitken. 2005. DNA damage to spermatozoa has impacts on fertilization and pregnancy. Cell and tissue research 322:33-41.

Lightowlers, R. N., P. F. Chinnery, D. M. Turnbull and N. Howell. 1997. Mammalian mitochondrial genetics: heredity, heteroplasmy and disease. Trends Genet 13:450-455.

Liot, G., B. Bossy, S. Lubitz, Y. Kushnareva, N. Sejbuk and E. Bossy-Wetzel. 2009. Complex II inhibition by 3-NP causes mitochondrial fragmentation and neuronal cell death via an NMDA- and ROS-dependent pathway. Cell death and differentiation 16:899-909.

Lu, A. L., X. Li, Y. Gu, P. M. Wright and D. Y. Chang. 2001. Repair of oxidative DNA damage: mechanisms and functions. Cell biochemistry and biophysics 35:141-70.

Lunt, D. H. and B. C. Hyman. 1997. Animal mitochondrial DNA recombination. Nature 387:247.

Lynch, M. 2010. Evolution of the mutation rate. Trends Genet 26:345-52.

Lynch, M., J. Blanchard, D. Houle, T. Kibota, S. Schultz, L. Vassilieva and J. Willis. 1999. Perspective: spontaneous deleterious mutation. Evolution 53:645-663.

Lynch, M., W. Sung, K. Morris, N. Coffey, C. R. Landry, E. B. Dopman, W. J. Dickinson, K. Okamoto, S. Kulkarni, D. L. Hartl and W. K. Thomas. 2008. A genome-wide view of the spectrum of spontaneous mutations in yeast. Proc Natl Acad Sci U S A 105:9272-7.

Martorell, L., D. G. Monckton, J. Gamez and M. Baiget. 2000. Complex patterns of male germline instability and somatic mosaicism in myotonic dystrophy type 1 . European journal of human genetics : EJHG 8:423-30.

Mattenberger, Y., D. I. James and J.-C. Martinou. 2003. Fusion of mitochondria in mammalian cells is dependent on the mitochondrial inner membrane potential and independent of microtubules or actin. FEBS Letters 538:53-59.

Medawar, P. B. 1952. An Unsolved Problem of Biology. Lewis, London.

Meeusen, S., J. M. McCaffery and J. Nunnari. 2004. Mitochondrial fusion intermediates revealed in vitro. Science (New York, N.Y.) 305:1747-52.

Meiklejohn, C. D., K. L. Montooth and D. M. Rand. 2007. Positive and negative selection on the mitochondrial genome. Trends in genetics : TIG 23:259-63. 
Meyer, J. N. and A. S. Bess. 2012. Involvement of autophagy and mitochondrial dynamics in determining the fate and effects of irreparable mitochondrial DNA damage. Autophagy 8.

Miceli, M. V., J. C. Jiang, A. Tiwari, J. F. Rodriguez-Quiñones and S. M. Jazwinski. 2011. Loss of mitochondrial membrane potential triggers the retrograde response extending yeast replicative lifespan. Frontiers in genetics 2:102.

Mishmar, D., E. Ruiz-Pesini, P. Golik, V. Macaulay, A. G. Clark, S. Hosseini, M. Brandon, K. Easley, E. Chen, M. D. Brown, R. I. Sukernik, A. Olckers and D. C. Wallace. 2003. Natural selection shaped regional mtDNA variation in humans. Proceedings of the National Academy of Sciences of the United States of America 100:171-6.

Mishmar, D., E. Ruiz-Pesini, M. Mondragon-Palomino, V. Procaccio, B. Gaut and D. C. Wallace. 2006. Adaptive selection of mitochondrial complex I subunits during primate radiation. Gene 378:11-18.

Mitra, K., C. Wunder, B. Roysam, G. Lin and J. Lippincott-Schwartz. 2009. A hyperfused mitochondrial state achieved at G1-S regulates cyclin $\mathrm{E}$ buildup and entry into $S$ phase. Proc Natl Acad Sci U S A 106:11960-11965.

Molina, A. J., J. D. Wikstrom, L. Stiles, G. Las, H. Mohamed, A. Elorza, G. Walzer, G. Twig, S. Katz, B. E. Corkey and O. S. Shirihai. 2009. Mitochondrial networking protects betacells from nutrient-induced apoptosis. Diabetes 58:2303-15.

Monaghan, P., N. B. Metcalfe and R. Torres. 2009. Oxidative stress as a mediator of life history trade-offs: mechanisms, measurements and interpretation. Ecol Lett 12:75-92.

Montooth, K. L., C. D. Meiklejohn, D. N. Abt and D. M. Rand. 2010. Mitochondrialnuclear epistasis affects fitness within species but does not contribute to fixed incompatibilities between species of Drosophila. Evolution 64:3364-3379.

Montooth, K. L. and D. M. Rand. 2008. The spectrum of mitochondrial mutation differs across species. PLoS Biol 6:e213.

Moraes, C. T. 2001. What regulates mitochondrial DNA copy number in animal cells? Trends Genet 17:199-205.

Morley, S. A., G. J. Lurman, J. N. Skepper, H. O. Portner and L. S. Peck. 2009. Thermal plasticity of mitochondria: a latitudinal comparison between Southern Ocean molluscs. Comp Biochem Physiol A Mol Integr Physiol 152:423-430. 
Mukai, T. 1964. The genetic structure of natural populations of Drosophila melanogaster. I. Spontaneous mutation rate of polygenes controlling viability. Genetics 50:1-19.

Muller, H. J. 1928. The Measurement of Gene Mutation Rate in Drosophila, Its High Variability, and Its Dependence upon Temperature. Genetics 13:279-357.

Murphy, M. P. 2009. How mitochondria produce reactive oxygen species. Biochemical Journal 417:1-13.

Okamoto, K. and J. M. Shaw. 2005. Mitochondrial morphology and dynamics in yeast and multicellular eukaryotes. Annu Rev Genet 39:503-536.

Ossowski, S., K. Schneeberger, J. I. Lucas-Lledó, N. Warthmann, R. M. Clark, R. G. Shaw, D. Weigel and M. Lynch. 2010. The rate and molecular spectrum of spontaneous mutations in Arabidopsis thaliana. Science (New York, N.Y.) 327:92-4.

Pak, J. W., A. Herbst, E. Bua, N. Gokey, D. McKenzie and J. M. Aiken. 2003. Rebuttal to Jacobs: The mitochondrial theory of aging: alive and well. Aging Cell 2:9-10.

Palermo, V., C. Falcone and C. Mazzoni. 2007. Apoptosis and aging in mitochondrial morphology mutants of S. cerevisiae. Folia Microbiol (Praha) 52:479-483.

Park, J. S., L. K. Sharma, H. Li, R. Xiang, D. Holstein, J. Wu, J. Lechleiter, S. L. Naylor, J. J. Deng, J. Lu and Y. Bai. 2009. A heteroplasmic, not homoplasmic, mitochondrial DNA mutation promotes tumorigenesis via alteration in reactive oxygen species generation and apoptosis. Human molecular genetics 18:1578-89.

Pascucci, B., M. D’Errico, E. Parlanti, S. Giovannini and E. Dogliotti. 2011. Role of nucleotide excision repair proteins in oxidative DNA damage repair: an updating. Biochemistry (Moscow) 76:4-15.

Pendergrass, W., N. Wolf and M. Poot. 2004. Efficacy of MitoTracker Green and CMXrosamine to measure changes in mitochondrial membrane potentials in living cells and tissues. Cytometry A 61:162-169.

Pham, N. A., T. Richardson, J. Cameron, B. Chue and B. H. Robinson. 2004. Altered mitochondrial structure and motion dynamics in living cells with energy metabolism defects revealed by real time microscope imaging. Microsc Microanal 10:247-260. 
Pletjushkina, O. Y., K. G. Lyamzaev, E. N. Popova, O. K. Nepryakhina, O. Y. Ivanova, L. V. Domnina, B. V. Chernyak and V. P. Skulachev. 2006. Effect of oxidative stress on dynamics of mitochondrial reticulum. Biochimica et biophysica acta 1757:518-24.

Prasad, A., M. J. F. Croydon-Sugarman, R. L. Murray and A. D. Cutter. 2011.

Temperature-dependent fecundity associates with latitude in Caenorhabditis briggsae. Evolution 65:52-63.

Raboin, M. J., A. F. Timko, D. K. Howe, M. A. Félix and D. R. Denver. 2010. Evolution of Caenorhabditis mitochondrial genome pseudogenes and Caenorhabditis briggsae natural isolates. Mol Biol Evol 27:1087-1096.

Raha, S. and B. H. Robinson. 2000. Mitochondria, oxygen free radicals, disease and ageing. Trends in biochemical sciences 25:502-8.

Richter, C., J. W. Park and B. N. Ames. 1988. Normal oxidative damage to mitochondrial and nuclear DNA is extensive. Proc Natl Acad Sci U S A 85:6465-6467.

Ross, J. A., D. C. Koboldt, J. E. Staisch, H. M. Chamberlin, B. P. Gupta, R. D. Miller, S. E. Baird and E. S. Haag. 2011. Caenorhabditis briggsae recombinant inbred line genotypes reveal inter-strain incompatibility and the evolution of recombination. PLoS genetics 7:e1002174. Public Library of Science.

Rossignol, R., B. Faustin, C. Rocher, M. Malgat, J. P. Mazat and T. Letellier. 2003. Mitochondrial threshold effects. Biochemical Journal 370:751-762.

Rowe, L. A., N. Degtyareva and P. W. Doetsch. 2008. DNA damage-induced reactive oxygen species (ROS) stress response in Saccharomyces cerevisiae. Free radical biology \& medicine 45:1167-77.

Rowe, L. A., N. Degtyareva and P. W. Doetsch. 2012. Yap1: a DNA damage responder in Saccharomyces cerevisiae. Mechanisms of ageing and development 133:147-56.

Russ, J. C. 2002. The Image Processing Handbook. 4th ed. CRC Press, LLC, Boca Raton.

Sato, M. and K. Sato. 2011. Degradation of paternal mitochondria by fertilizationtriggered autophagy in C. elegans embryos. Science (New York, N.Y.) 334:1141-4.

Schon, E. A., S. Dimauro, M. Hirano and R. W. Gilkerson. 2010. Therapeutic prospects for mitochondrial disease. Trends Mol Med, doi: S1471-4914(10)00058-4 [pii] 10.1016/j.molmed.2010.04.007. 
Schon, E. A. and G. Manfredi. 2003. Neuronal degeneration and mitochondrial dysfunction. J Clin Invest 111:303-312.

Scorrano, L. 2005. Proteins that fuse and fragment mitochondria in apoptosis: confissing a deadly con-fusion? Journal of bioenergetics and biomembranes 37:165-70.

Sedensky, M. M. and P. G. Morgan. 2006. Mitochondrial respiration and reactive oxygen species in mitochondrial aging mutants. Exp Gerontol 41:237-245.

Selman, C., J. D. Blount, D. H. Nussey and J. R. Speakman. 2012. Oxidative damage, ageing, and life-history evolution: where now? Trends in ecology \& evolution null.

Senoo-Matsuda, N., K. Yasuda, M. Tsuda, T. Ohkubo, S. Yoshimura, H. Nakazawa, P. S. Hartman and N. Ishii. 2001. A defect in the cytochrome b large subunit in complex II causes both superoxide anion overproduction and abnormal energy metabolism in Caenorhabditis elegans. J Biol Chem 276:41553-41558.

Shanks, M. E., C. A. May, Y. E. Dubrova, P. Balaresque, Z. H. Rosser, S. M. Adams and M. A. Jobling. 2008. Complex germline and somatic mutation processes at a haploid human minisatellite shown by single-molecule analysis. Mutation research 648:46-53.

Shibutani, S., M. Takeshita and A. P. Grollman. 1991. Insertion of specific bases during DNA synthesis past the oxidation-damaged base 8-oxodG. Nature 349:431-4.

Shigenaga, M. K., T. M. Hagen and B. N. Ames. 1994. Oxidative damage and mitochondrial decay in aging. Proc Natl Acad Sci U S A 91:10771-10778.

Shokolenko, I., N. Venediktova, A. Bochkareva, G. Wilson and M. F. Alexeyev. 2009. Oxidative stress induces degradation of mitochondrial DNA. Nucleic acids research 37:2539-48.

Song, Z., H. Chen, M. Fiket, C. Alexander and D. C. Chan. 2007. OPA1 processing controls mitochondrial fusion and is regulated by mRNA splicing, membrane potential, and Yme1L. J Cell Biol 178:749-755.

Stewart, J. B., C. Freyer, J. L. Elson, A. Wredenberg, Z. Cansu, A. Trifunovic and N.-G. G. Larsson. 2008. Strong purifying selection in transmission of mammalian mitochondrial DNA. PLoS biology 6:e10. Public Library of Science.

Stiernagle, T. 2006. Maintenance of C. elegans. Pp. 1-11 in I. A. Hope, ed. C. elegans: A Practical Approach. Oxford University Press, New York, NY. 
Stoltzfus, A. 2008. Evidence for a predominant role of oxidative damage in germline mutation in mammals. Mutation research 644:71-3.

Su, B., X. Wang, L. Zheng, G. Perry, M. A. Smith and X. Zhu. 2010. Abnormal mitochondrial dynamics and neurodegenerative diseases. Biochimica et biophysica acta 1802:135-42.

Sudhaus, W. and K. Kiontke. 2007. Comparison of the cryptic nematode species Caenorhabditis brenneri sp. n. and C. remanei (Nematoda: Rhabditidae) with the stem species pattern of the Caenorhabditis Elegans group. Zootaxa 1456:45-62.

Swartzlander, D. B., L. M. Griffiths, J. Lee, N. P. Degtyareva, P. W. Doetsch and A. H. Corbett. 2010. Regulation of base excision repair: Ntg1 nuclear and mitochondrial dynamic localization in response to genotoxic stress. Nucleic acids research 38:3963-74.

Trifunovic, A., A. Hansson, A. Wredenberg, A. T. Rovio, E. Dufour, I. Khvorostov, J. N. Spelbrink, R. Wibom, H. T. Jacobs and N. G. Larsson. 2005. Somatic mtDNA mutations cause aging phenotypes without affecting reactive oxygen species production. Proc Natl Acad Sci U S A 102:17993-17998.

Trimmer, P. A., R. H. Swerdlow, J. K. Parks, P. Keeney, J. P. Bennett Jr., S. W. Miller, R. E. Davis and W. D. Parker Jr. 2000. Abnormal mitochondrial morphology in sporadic Parkinson's and Alzheimer's disease cybrid cell lines. Exp Neurol 162:37-50.

Tsang, W. Y. and B. D. Lemire. 2002. Stable heteroplasmy but differential inheritance of a large mitochondrial DNA deletion in nematodes. Biochem Cell Biol 80:645-654.

Tuma, R. 2001. The two faces of oxygen. Sci Aging Knowledge Environ 2001:oa5.

Tuppen, H. A., E. L. Blakely, D. M. Turnbull and R. W. Taylor. 2010. Mitochondrial DNA mutations and human disease. Biochim Biophys Acta 1797:113-128.

Twig, G., A. Elorza, A. J. Molina, H. Mohamed, J. D. Wikstrom, G. Walzer, L. Stiles, S. E. Haigh, S. Katz, G. Las, J. Alroy, M. Wu, B. F. Py, J. Yuan, J. T. Deeney, B. E. Corkey and O. S. Shirihai. 2008a. Fission and selective fusion govern mitochondrial segregation and elimination by autophagy. EMBO J 27:433-446.

Twig, G., B. B. Hyde and O. S. Shirihai. 2008b. Mitochondrial fusion, fission and autophagy as a quality control axis: the bioenergetic view. Biochim Biophys Acta 1777:1092-1097. 
Twig, G. and O. S. Shirihai. 2011. The interplay between mitochondrial dynamics and mitophagy. Antioxidants \& redox signaling 14:1939-51.

Valavanidis, A., T. Vlachogianni and C. Fiotakis. 2009. 8-hydroxy-2' -deoxyguanosine (8OHdG): A critical biomarker of oxidative stress and carcinogenesis. Journal of environmental science and health. Part C, Environmental carcinogenesis \& ecotoxicology reviews 27:120-39. Taylor \& Francis.

Vander Heiden, M. G., N. S. Chandel, X. X. Li, P. T. Schumacker, M. Colombini and C. B. Thompson. 2000. Outer mitochondrial membrane permeability can regulate coupled respiration and cell survival. Proceedings of the National Academy of Sciences of the United States of America 97:4666-71.

Velando, A., R. Torres and C. Alonso-Alvarez. 2008. Avoiding bad genes: oxidatively damaged DNA in germ line and mate choice. BioEssays : news and reviews in molecular, cellular and developmental biology 30:1212-9.

Ventura, N., S. L. Rea and R. Testi. 2006. Long-lived C. elegans Mitochondrial mutants as a model for human mitochondrial-associated diseases. Exp Gerontol 41:974-991.

Verkaart, S., W. J. Koopman, S. E. van Emst-de Vries, L. G. J. Nijtmans, L. W. P. J. van den Heuvel, J. A. M. Smeitink and P. H. G. M. Willems. 2007. Superoxide production is inversely related to complex I activity in inherited complex I deficiency. Biochimica Et Biophysica Acta-Molecular Basis of Disease 1772:373-381.

Vijg, J. 2000. Somatic mutations and aging: a re-evaluation. Mutation Research/Fundamental and Molecular Mechanisms of Mutagenesis 447:117-135.

Vijg, J. and M. E. T. Dollé. 2002. Large genome rearrangements as a primary cause of aging. Mechanisms of ageing and development 123:907-15.

Wallace, D. C. 2005a. A mitochondrial paradigm of metabolic and degenerative diseases, aging, and cancer: a dawn for evolutionary medicine. Annu Rev Genet 39:359-407.

Wallace, D. C. 1999. Mitochondrial diseases in man and mouse. Science 283:1482-1488.

Wallace, D. C. 2005b. The mitochondrial genome in human adaptive radiation and disease: on the road to therapeutics and performance enhancement. Gene 354:169180. 
Wanagat, J., Z. Cao, P. Pathare and J. M. Aiken. 2001. Mitochondrial DNA deletion mutations colocalize with segmental electron transport system abnormalities, muscle fiber atrophy, fiber splitting, and oxidative damage in sarcopenia. FASEB J 15:322-332.

Wang, X., B. Su, H. Fujioka and X. Zhu. 2008. Dynamin-like protein 1 reduction underlies mitochondrial morphology and distribution abnormalities in fibroblasts from sporadic Alzheimer's disease patients. Am J Pathol 173:470-82.

Wang, Y., Y. Nartiss, B. Steipe, G. A. McQuibban and P. K. Kim. 2012. ROS-induced mitochondrial depolarization initiates PARK2/PARKIN-dependent mitochondrial degradation by autophagy. Autophagy 8:14-13.

Waters, J. C. 2009. Accuracy and precision in quantitative fluorescence microscopy. J Cell Biol 185:1135-1148.

Wei, Y. H. and H. C. Lee. 2002. Oxidative stress, mitochondrial DNA mutation, and impairment of antioxidant enzymes in aging. Exp Biol Med (Maywood) 227:671-682.

Westermann, B. 2010. Mitochondrial dynamics in model organisms: What yeasts, worms and flies have taughtus about fusion and fission of mitochondria. Seminars in Cell \& Developmental Biology 21:542-549.

Wikstrom, J. D., G. Twig and O. S. Shirihai. 2009. What can mitochondrial heterogeneity tell us about mitochondrial dynamics and autophagy? The international journal of biochemistry cell biology 41:1914-1927. Elsevier Ltd.

Willett, C. S. and R. S. Burton. 2004. Evolution of interacting proteins in the mitochondrial electron transport system in a marine copepod. Molecular biology and evolution 21:443-53.

Winklhofer, K. F. and C. Haass. 2010. Mitochondrial dysfunction in Parkinson's disease. Biochim Biophys Acta 1802:29-44.

Winterbourn, C. C. 2008. Reconciling the chemistry and biology of reactive oxygen species. Nature chemical biology 4:278-86. Nature Publishing Group.

Wolff, J. N., S. Gandre, A. Kalinin and N. J. Gemmell. 2008. Delimiting the frequency of paternal leakage of mitochondrial DNA in chinook salmon. Genetics 179:1029-32. 
Yakes, F. M. and B. Van Houten. 1997. Mitochondrial DNA damage is more extensive and persists longer than nuclear DNA damage in human cells following oxidative stress. Proc Natl Acad Sci U S A 94:514-519.

Yang, W., J. Li and S. Hekimi. 2007. A Measurable increase in oxidative damage due to reduction in superoxide detoxification fails to shorten the life span of long-lived mitochondrial mutants of Caenorhabditis elegans. Genetics 177:2063-2074.

Yasuda, K., H. Adachi, Y. Fujiwara and N. Ishii. 1999. Protein Carbonyl Accumulation in Aging Dauer Formation--Defective (daf) Mutants of Caenorhabditis elegans. The Journals of Gerontology Series A: Biological Sciences and Medical Sciences 54:B47-B51.

Yasuda, K., P. S. Hartman, T. Ishii, H. Suda, A. Akatsuka, T. Shoyama, M. Miyazawa and N. Ishii. 2011. Interrelationships between mitochondrial fusion, energy metabolism and oxidative stress during development in Caenorhabditis elegans. Biochemical and biophysical research communications 404:751-5.

Zhou, Q., H. Li and D. Xue. 2011. Elimination of paternal mitochondria through the lysosomal degradation pathway in C. elegans. Cell research 21:1662-9.

Zielonka, J. and B. Kalyanaraman. 2010. Hydroethidine- and MitoSOX-derived red fluorescence is not a reliable indicator of intracellular superoxide formation: another inconvenient truth. Free Radic Biol Med 48:983-1001.

Zouros, E. 1994. An Unusual Type of Mitochondrial DNA Inheritance in the Blue Mussel Mytilus. Proceedings of the National Academy of Sciences 91:7463-7467. 


\section{APPENDIX}

\section{Description of mitochondrial morphology analysis}

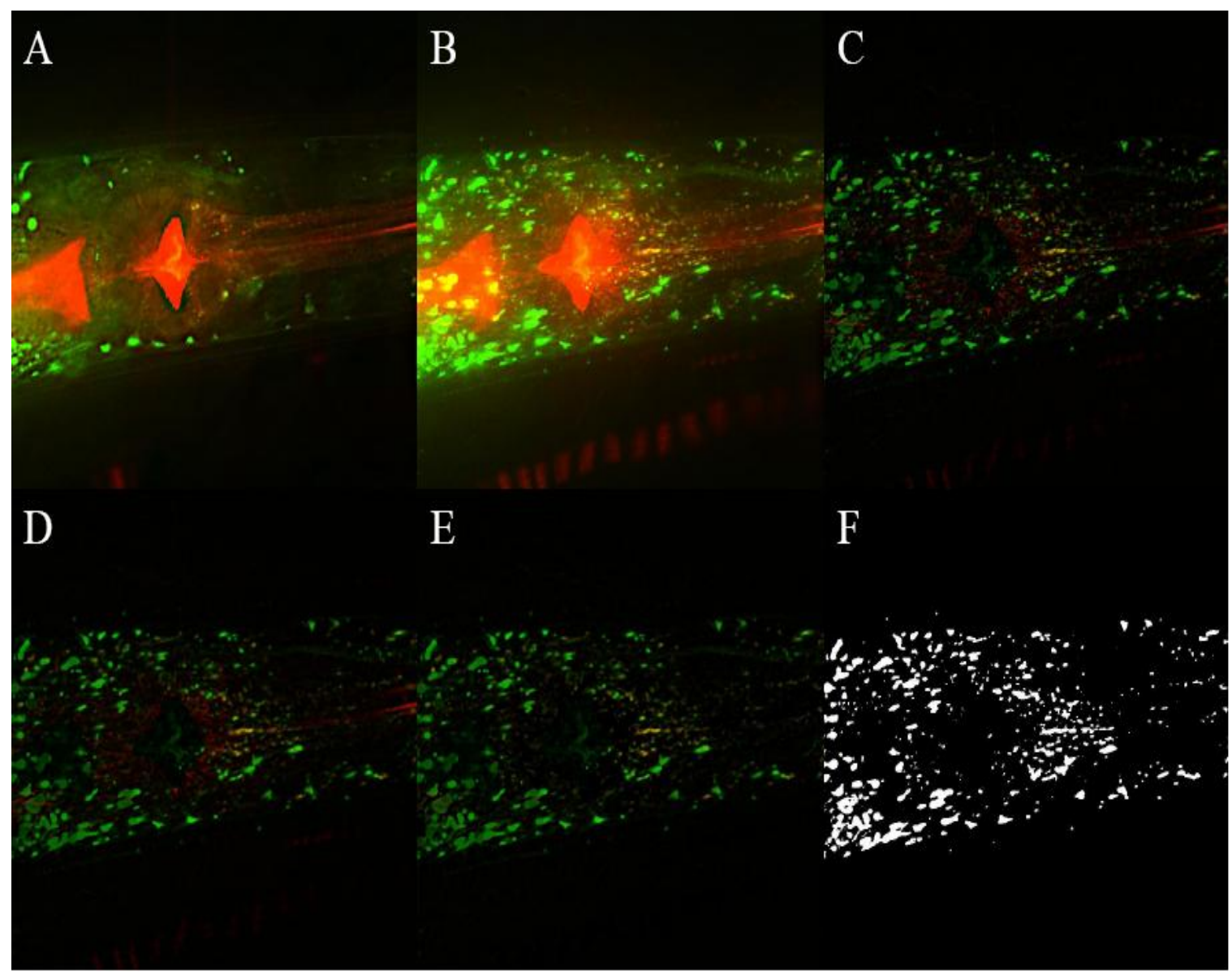

Figure A.1 - Depiction of mitochondrial morphology analysis process. A. One slice of representative image of a nematode labeled with MitoTracker Red CMXRos and MitoTracker Green FM B. Conflated image wherein each pixel represents the maximum fluorescence intensity of all slices. C. 7x7 tophat filtered image of B. D. Median filter applied to image C. E. Thresholded image of green channel of mitochondria (red channel not shown, but A-D show both red and green together). F. Binary image of E. Image J was used to enclose each object in the pharyngeal bulb area of the binary image (F) for both the red and green channels, and subsequently used to calculate the shape descriptors outlined in Table 2.1 and page 28. Shape descriptors pertaining to objects from the red channel were denoted as functional mitochondrial traits, and those from the green channel were denoted as non-functional. For each sample, the average of each shape descriptor was used for subsequent analysis (i.e., the average 2-dimensional area of each nonfunctional object within one sample was used to calculate that sample's measure of $A_{N}$ ). 